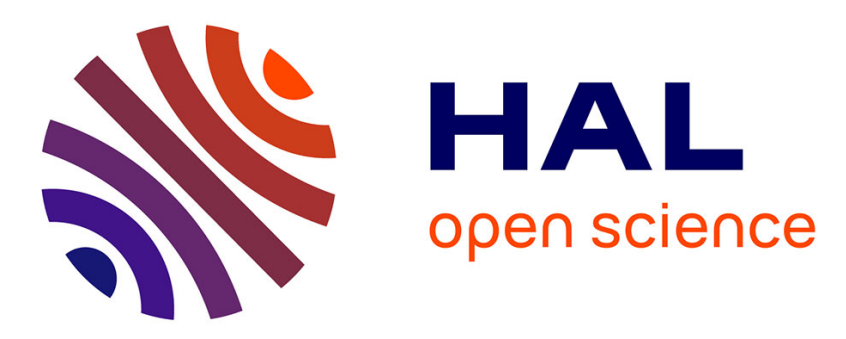

\title{
Design of hybrid nanovehicles for remotely triggered drug release: an overview
}

Ji Liu, Christophe Detrembleur, Stéphane Mornet, Christine Jérôme, Etienne Duguet

\section{- To cite this version:}

Ji Liu, Christophe Detrembleur, Stéphane Mornet, Christine Jérôme, Etienne Duguet. Design of hybrid nanovehicles for remotely triggered drug release: an overview. Journal of materials chemistry B, 2015, 3 (30), pp.6117-6147. 10.1039/C5TB00664C . hal-01180261

\section{HAL Id: hal-01180261 https://hal.science/hal-01180261}

Submitted on 12 Feb 2021

HAL is a multi-disciplinary open access archive for the deposit and dissemination of scientific research documents, whether they are published or not. The documents may come from teaching and research institutions in France or abroad, or from public or private research centers.
L'archive ouverte pluridisciplinaire HAL, est destinée au dépôt et à la diffusion de documents scientifiques de niveau recherche, publiés ou non, émanant des établissements d'enseignement et de recherche français ou étrangers, des laboratoires publics ou privés. 


\title{
Design of Hybrid Nanovehicles for Remotely Triggered Drug Release:
}

\section{An Overview}

\author{
Ji Liu, ${ }^{a, b \dagger}$ Christophe Detrembleur, ${ }^{a}$ Stéphane Mornet ${ }^{b}$ Christine Jérôme*a and Etienne Duguet ${ }^{* b}$ \\ Received (in $X X X, X X X)$ Xth $X X X X X X X X X 20 X X$, Accepted $X$ th $X X X X X X X X X 20 X X$ \\ ${ }_{5}$ DOI: 10.1039/b000000x
}

In the past few decades, various nanovehicles have been developed as drug delivery systems, in which inorganic and organic components are integrated within a nano-object. Upon the application of remote stimuli, e.g. alternating magnetic field, near infrared or ultrasound radiations, the release of guest molecules can be triggered in a quite controlled manner. Herein, we review different hybrid

10 nanostructures which have already been reported for the remotely triggered release, such as those based on (1) host/guest conjugates, (2) core/corona nanoparticles, (3) polymer nanogels, (4) polymer micelles, (5) liposomes, (6) mesoporous silica particles and (7) hollow nanoparticles. Moreover, we also summarize six underlying mechanisms that govern such kind of remotely triggered release behaviours: (1) enhanced diffusion and/or permeation, (2) thermo- or photo-labile bond cleavage, (3) fusion of phase15 changed materials, (4) photo-induced isomerisation, (5) thermo-induced swelling/de-swelling of thermoresponsive polymer, and (6) destruction of the nanostructures. The ways in which different components are incorporated into an integrated hybrid nanostructure and how they contribute to the remotely triggered release behaviours are detailed.

\section{Introduction}

20 Application of traditional anti-cancer chemotherapeutic reagents is still circumvented by their poor water solubility, metabolic instability, as well as their dose-dependent toxic potential. This is why substantial amount of effort has been devoted toward developing innovative drug delivery system (DDS) to efficiently 25 and effectively transport such chemotherapeutic reagents. ${ }^{1}$ Controlled drug delivery systems, which are intended to deliver drugs at predetermined rates within a predefined period, have been developed to overcome the shortcomings of conventional chemotherapeutic modulations. As shown in Fig. 1, the annual 30 number of articles aiming to develop DDS has substantially increased in the last decade. Due to this huge amount of publications on DDS, it is impossible to review all of them, thus we selected those, which are the most original and/or promising systems according to our own experience. Moreover this review 35 is especially focused on delicately-designed hybrid nanovehicles specifically for remotely triggered drug release.

\section{1. Why remote triggers?}

In order to address those complicated issues emerging from 40 the clinical practices, an ideal DDS must satisfy some "selfcontradictory" requirements. It should primarily be capable of safely delivering high-amount of chemotherapeutic agents to the

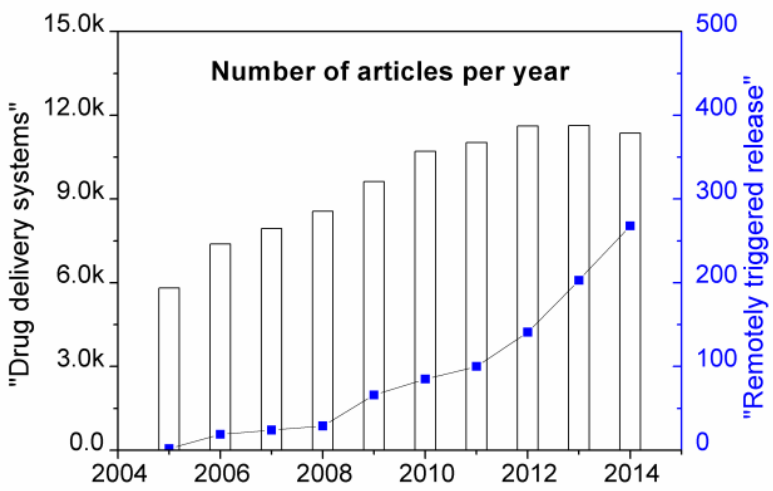

Fig. 1 Number of articles per year from 2006 to 2015 on "drug delivery 45 systems" (left) and "remotely triggered release" (right), data obtained from Scopus database on June $3^{\text {rd }} 2015$.

disease location, with a "zero premature release" character during the in vivo transport. On the other hand, controlled release of the chemotherapeutic payloads should be easily achieved in a 50 controlled manner, upon the application of specific stimuli. ${ }^{2,3}$ Usually, this kind of stimuli can be present in vivo or remotely applied by clinicians. For the former one, some disease microenvironments might manifest themselves with particularly different physiological characteristics from their healthy 55 surroundings, such as difference in $\mathrm{pH}$, concentration of glucose 
or reductive reagents, presence of a specific enzyme, etc. ${ }^{4}$ Thus, once the nanovehicles reach those disease pathologies and experience such pathological difference, it might allow them to respond specifically to these "triggers" with chemical/physical 5 changes, following with the subsequent drug release. For example, the extracellular $\mathrm{pH}$ in solid tumours tends to be acidic (ca.6.5-6.0), compared to that of the vascular fluid (7.4). Additionally, the $\mathrm{pH}$ value decreases along the cellular uptake of nanovehicles from 7.4 (cytosolic) to 5.5-6.0 (endosomes), and 10 finally reaches $c a$. 5.0 (lysosomes). ${ }^{3,5}$ Therefore, DDS bearing $\mathrm{pH}$-sensitive moieties, like acid-labile bonds, could be disintegrated or become permeable after reaching the tumour sites and/or internalizing within the lesion cells, resulting in the $\mathrm{pH}-$ induced release of chemotherapeutic payloads. Up to now, many 15 efforts have been directed to developing DDS with the potential to release the cargo molecules in response to these internal pathological stimuli. ${ }^{6-8}$

Although significant progress has been achieved on DDS sensitive to these pathological "triggers", such kind of release 20 behaviours are still executed in a passive mode and lack of control over the dose, timing and duration of the drug release. ${ }^{9}$ Moreover, this approach could not allow for pulsatile or selfregulated drug release, which is adjusted to the staging of biological rhythms, since the onset of some diseases always 25 exhibit strong circadian temporal dependence, such as diabetes. ${ }^{10}$ Therefore, it would be more desirable if the chemotherapeutic payloads could be delivered in a manner that precisely matches the physiological needs at a proper period and/or within a specific site. Fortunately, these drawbacks could be well overcome with 30 manually-introduced remote stimuli, e.g. ultrasound, magnetic field or light application, due to the facile accessibility and manipulative controllability, as well as less side-effects, making them appealing in future DDS developments. ${ }^{11}$ As summarized in Fig. 1, there is also a continuous increase in the number of 35 articles per year dealing with "remotely triggered release". Popularity of the "remotely triggered release" concept also results in the constant emerging of delicately-designed nanovehicles, bearing functional moieties sensitive to a certain remote stimuli. Up to now, structures based on superparamagnetic nanoparticles 40 with the responsiveness to magnetic fields, ${ }^{12-14}$ gold nanoparticles to light, ${ }^{15}, 16$ liposomes to high-frequency ultrasound, ${ }^{17-19}$ etc, have ever been thoroughly reviewed for remotely triggered drug release. In addition to the "remotely triggered release" function, these intricate nanostructures also bring out some 45 chances/possibilities endow the DDS with other credentials, for example, in vivo tracking with the aid of biomedical imaging, ${ }^{20}$ which further demonstrate their potential in biomedical application.

\section{1. 2. Why nanovehicles?}

Intravenous administration is one of the most commonly used procedures in those clinical practices, consisting of the perfusion of a solution or dispersion directly into a vein. Compared with other routes of administration, the intravenous route is the fastest 55 way to deliver fluids containing chemotherapeutic reagents throughout the body. Generally, a successful DDS for intravenous administration should possess good biocompatibility, high colloidal stability, efficient protection and entrapment of the chemotherapeutics, as well as improved pharmacokinetics. On 60 the other hand, it is also required to be capable to escape the mononuclear phagocyte system (MPS) and target to the desirable sites with a high efficiency, leading to an improved therapeutic treatment. ${ }^{21}$ Nowadays, rapid progress in nanotechnology offers novel insights and concepts for DDS, and also arises a growing ${ }_{65}$ interest in nano-sized DDS. ${ }^{22}$ As reported by Matsumura and coll., ${ }^{23}$ nanoparticles are able to spontaneously accumulate in some pathological sites, e.g. tumours, via the well-established enhanced permeability and retention (EPR) effect. This effect involves the extravasation and accumulation of those 70 nanoparticles within the interstitial tumour space, through the "leaky" but discontinuous pathological neovasculatures within the tumour tissues. Thus, this EPR effect allows for a passive targeting of nanovehicles to tumours, based on the specific cutoff size of those leaky vasculatures.

75 On the other hand, some nanoparticles are reported to exhibit characteristic responsiveness to those remote triggers as-referred. For examples, gold nanoparticles with different shapes (nanorods, nanocages, nanoshells, etc.), exhibiting a longitudinal surface plasmon resonance (SPR) band in the near infrared (NIR) region, 80 are sensitive to NIR light, superparamagnetic nanoparticles (magnetite or maghemite) exhibit intrinsic sensitiveness to external magnetic fields without remnant magnetization, and nano-sized liposomes, made of thermo-sensitive lipids, are able to undergo a phase change upon exposure to high-frequency 85 ultrasound, etc. Vehicles made of such kind of nanoparticles, bearing responsiveness to these remote triggers, show a promising application as DDS for remotely triggered release. Up to now, some general reviews dealing with the biomedical application of these nanoparticles, have been reported, such as the 90 one of Duguet and coll. ${ }^{24}$ on magnetic nanoparticles, Murphy and coll. ${ }^{16}$ on gold nanorods, Halas and coll. ${ }^{15}$ on gold nanoshells, Xia and coll. ${ }^{25}$ on gold nanocages, Perrie and coll. ${ }^{26}$ and Muller and coll. ${ }^{27}$ on thermo-sensitive liposomes, to cite a few. Therefore, the high-efficacy accumulation of nanoparticles within 95 the pathological sites, as well as their responsiveness to remote stimuli, promises their application as DDS for chemotherapeutic payloads, with potency of remotely triggered release.

\section{3. Why hybrid structures?}

100 Various structures, e.g. polymer micelles or nanogels, ${ }^{28}$ mesoporous silica, ${ }^{2}$ liposomes, ${ }^{26}$ as well as their hybrid derivatives, ${ }^{6}$ have been exploited for DDS. The attractiveness of those hybrid structures, differentiating themselves form other mono-component structures, arises from the synergistic 105 combination of different functions, which are interesting by themselves but become more appealing after integration within a nano-sized object. ${ }^{29}$ Preponderant literatures on such hybrid structures are mostly focused on the integration of organic components, e.g. biodegradable macromolecules, stimuli110 responsive macromolecules, amphiphilic macromolecules, lipids and dendrimers, with inorganic ingredients, e.g. gold, iron oxide, silver, nanocrystals and silica nanoparticles. ${ }^{6}$ The selection of different components to self-assemble within one hybrid body is a tricky task, and it is usually decided by the envisioned 115 therapeutic goal, type of payloads, biocompatible/biocompatible essence of each component, as well as the expected routes for 


\section{Cite this: DOI: 10.1039/c0xx00000x}

\section{www.rsc.org/xxxxxx}

ARTICLE TYPE

administration, etc. Being synergistic, each component benefits from the combination within the single body. For those inorganic nanoparticles, taking maghemite nanoparticles as an example, it might suffer from a lack of colloidal stability in biological fluids 5 and would be rapidly cleared from the bloodstream before reaching the targeted pathological sites. ${ }^{30}$ In order to overcome these obstacles, surface coating or encapsulation within some compatible nanovehicles is preferred, especially when it allows introducing several other supplementary functions. Over the 10 years, various synthetic, structural, physicochemical and biomedical aspects of magnetic hybrid nanostructures have been discussed in a number of excellent reviews, such as the one by Muller and coll. ${ }^{31}$ on magnetic liposomes, Brazel and coll. ${ }^{12}$ on thermo-responsive magnetic vehicles, Knezenic and coll. ${ }^{32}$ on 15 magnetic mesoporous silica and Lecommandoux and coll. ${ }^{13}$ on magnetic composites, to cite a few.

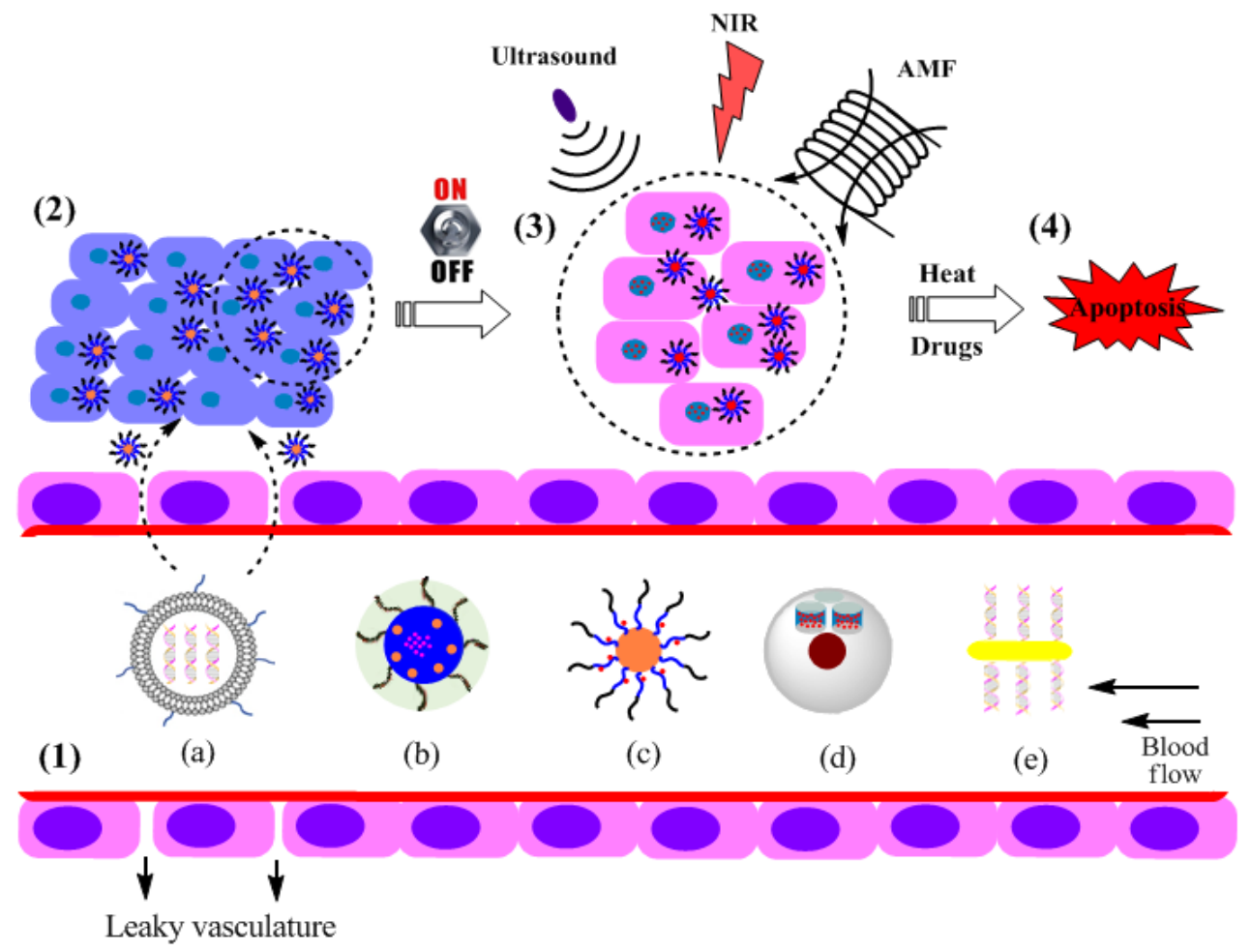

Scheme: (1) Hybrid nanovehicles with different kinds of structures designed for drug delivery systems, such as liposomes (a), cross-linked polymer 20 nanogels or micelles (b), organic/inorganic core/corona nanohybrids (c), mesoporous silica-based nanovehicles engineered with gating moieties (d) and host/guest nano-conjugates (e); (2) delivery of the guest molecules to desirable tumour sites via passive or active targeting through the vasculature; (3) trigger release of the loaded cargos through remote stimuli, e.g. ultrasound, light (such as near infrared laser) or alternating magnetic filed (AMF); (4) tumour cell apoptosis resulting from the synergistic effect of thermal and chemotherapeutic contributions.

\section{1. 4. Why this review?}

The field of remotely-triggered drug release has already been covered by some pioneering reviews. The reviews by Kohane and coll. on "remotely triggerable drug delivery systems" 20 and "Materials to clinical devices: technologies for remotely triggered 30 drug delivery", ${ }^{33}$ dedicated most on the classification of different remote triggers as well as those different structures possessing the potency for triggered release corresponding to those remote triggers. In the present review, we systematically discuss those hybrid vehicles within nano-scaled dimension, according to 35 different mechanisms for remotely-triggered drug release, such as covalent bond cleavage, enhanced diffusion and permeation, fusion of phase-change materials, etc. It is also different from those recent reviews dealing with one trigger, as well as the reviews focusing on both mono-component and hybrid structures 40 in the scale of micro- and nano-dimension, such as Lecommandoux and coll. ${ }^{13}$ on magnetic-responsive polymer composites, Almutairi and coll. ${ }^{34}$ on light (UV and NIR)triggered release from nanovehicles based on both monocomponent and hybrid composites, Perrie and coll. ${ }^{26}$ on locally 45 and remotely triggered release from mono-component or hybrid liposome-based systems, Couvreur and coll. ${ }^{35}$ on both locally and remotely triggered release from nano-carriers, etc. 
Here, we summarize the updated state-of-the-art researches on hybrid nanovehicles developed for remotely-triggered release (Scheme 1), while for other DDS, which do not match these criteria, like those mono-componented DDS, macro/microscaled ${ }_{5}$ DDS, DDS for remotely triggers via controversial UV irradiation, etc., will not be covered in the present review. Classification of those hybrid nanovehicles will be first highlighted according to different nanostructures. Even though different hybrid nanostructures might have ever been discussed in some of those 10 above-mentioned reviews, herein, we will only summarize the hybrid nanostructures, which are indeed designed for remotelytriggered release. Moreover, special insights will be, for the first time, as far as we know, dedicated into the underlying mechanisms of the remotely-triggered release from those 15 delicately-designed hybrid nanovehicles.

\section{Remote triggers for DDS}

\section{1. Alternating magnetic field (AMF)}

Magnetic nanoparticles, especially uniform-sized and highly20 crystalline superparamagnetic iron oxide nanoparticles (maghemite or magnetite), have found tremendous applications in the biomedical field, including biological sensing, intracellular DDS, magnetic hyperthermia therapy and medical diagnosis, due to their tuneable nanomagnetism and superior biocompatibility. 25 24, $36 \mathrm{Up}$ to now, magnetite and maghemite nanoparticles have reached clinical trials as high-efficient $T_{2}$ contrast agents for magnetic resonance imaging (MRI). Moreover, upon exposure to external AMF, a local heating is generated via the mechanism of Néel relaxation, Brownian relaxation or hysteresis losses. ${ }^{24}$ ${ }_{30}$ Usually, tumour cells might undergo heat stress in the range of $41-46^{\circ} \mathrm{C}$, resulting in some intra- and extra-cellular degradation processes, e.g. protein denaturation. Thus, magnetic nanoparticles accumulated within the tumour sites could act as heating mediators, promising the use of magnetic hyperthermia for 35 tumours ablation. ${ }^{36}$ Nevertheless, concentration of the magnetic nanoparticles in the tumours cells should be high enough to accomplish an efficient and homogeneous local heating. That is why, the clinical development of magnetic hyperthermia remains rare at this moment. However, on the other hand, this kind of 40 heating capability could be, and probably more easily, exploited to increase the local temperature of the DDS. Taking account of the specific heating capability, magnetic nanovehicles could be integrated with some other thermo-responsive moieties, such as phase-changed materials, thermo-labile covalent bond, thermo45 responsive macromolecules, etc, in order to realize the release of chemotherapeutic payloads via remote AMF activation. ${ }^{37}$ Moreover, The combination of magnetic nanoparticles and thermo-responsive hydrogels or lipids for remotely AMFtriggered release has been discussed within the reviews by Brazel 50 and coll. ${ }^{12}$ and Liu and coll. ${ }^{14}$ The designing and use of magnetic hybrid nanovehicles for remotely AMF-triggered release are discussed in the following sections.

\section{2. Near infrared (NIR) light}

55 Light stimulus is one of the most attractive remote triggers, since it can be remotely introduced with high spatial and temporal precision. Minimization of light absorption could be accomplished by adjusting the irradiation wavelength within the biologically-friendly window, that is, the near infrared (NIR) part 60 of the spectrum $(650-950 \mathrm{~nm})$. In such a wavelength range, blood and tissues are maximally transparent and the NIR light could penetrate deeply and safely into the tissues by few centimeters, promising a further in vivo application. ${ }^{38}$ Plasmonic metallic nanoparticles, such as gold nanorods, nanocages and nanoshells, 65 exhibit a strong absorption in the NIR region due to the surface plasmon resonance (SPR) oscillations, while the SPR band is usually defined by some structural parameters, such as aspect ratio, size, shell thickness, etc. ${ }^{39}$ On account of their extremely low-levelled photoemission quantum yield and large extinction 70 coefficient at the SPR wavelength, such kind of gold nanoparticles (GNPs) could convert optical energy into thermal energy with a high efficiency upon NIR irradiation. ${ }^{16}$ In addition to the light-sensitivity, GNPs also manifest themselves with some attractive intrinsic properties, such as biocompatible essence, 75 facile fabrication, easy functionalization with a wide variety of host-carrier molecules, as well as convenient conjugation with different guest molecules, etc. On the other hand, taking advantage of their nanometric size, GNPs could also passively accumulate within the tumour sites through the EPR 80 mechanism. ${ }^{23}$ Therefore, through elaborated optimization on those variables, including shape, size, concentration of GNPs within the tumour sites, coupled with the NIR irradiation power and period, etc., precise and localized tumour ablation via NIRinduced phototherapy could be easily realized. ${ }^{40}$ Similarly to the 85 magnetic hyperthermia, the NIR-induced photothermia could also be used to remotely trigger the release of chemotherapeutic payloads, once GNPs are integrated within a DDS. Up to now, there exists also some reviews dedicated to the biomedical applications (including remotely NIR-triggered release) of GNPs, 90 such as the one reported by Halas and coll. ${ }^{41}$ on gold nanoshells, Murphy and coll. ${ }^{16}$ on gold nanorods, and Xia and coll. ${ }^{42}$ on gold nanocages, etc. The designing and use of GNPs-bearing hybrid nanovehicles for remotely NIR-triggered release are discussed in the following sections.

95

\section{3. Ultrasound}

Ultrasound has also found many applications in clinical practices, such as ultrasound imaging, tumour and fibroid ablation, kidney-stone shattering, etc. ${ }^{17}$ Similarly to the former 100 NIR light, the focused ultrasound could also be precisely introduced with a good spatial and temporal control, but with a much deeper penetration compared to NIR light. ${ }^{43}$ The absorption of ultrasonic energy results in a rise of the local temperature, namely, ultrasound hyperthermia, and it could also be used 105 complementarily with chemotherapeutic treatments. ${ }^{19}$ On the other side, this kind of ultrasound hyperthermia and/or ultrasound-induced cavitations could also be exploited to trigger the cargo release from DDS, such as polymeric assemblies, nonthermo-responsive or thermo-responsive liposomes, mesoporous 110 silica, etc. ${ }^{26}$ Moreover, ultrasound exposure alone has been proven to improve the cell membrane permeability, thus, facilitates the entrance of exotic modalities into the cells. ${ }^{44}$ These characteristics make ultrasound technique particularly promising in DDS, not only because the application of ultrasound can 


\section{Cite this: DOI: $10.1039 / \mathrm{c0xx00000x}$}

\section{www.rsc.org/xxxxxx}

ARTICLE TYPE

accelerate the uptake of nanovehicles through the cellular membranes, but also in vivo trigger the release of drug payloads, synergistically contributing to an improved therapeutic efficiency. ${ }^{17}$

5 Generally, ultrasound technology includes low-frequency ultrasound and high-frequency diagnostic ultrasound, ${ }^{18}$ but the wave frequency might remarkably impact the subsequent release behaviours, thus of interest as a remote trigger. Taking liposome as an example, low-frequency ultrasound with a longer 10 wavelength is in favour of forming transient pores, due to the packing rearrangement of the lipid molecules. Thus, triggered release behaviours in this case could be attributed to the enhanced permeability of the phospholipid bilayers. ${ }^{19}$ While for highfrequency ultrasound, due to the high intensity in the focal spot, 15 cavitations at higher acoustical pressures will induce more violent structural oscillation, eventually resulting in the destruction of those vehicles, once local pressure exceeds the elastic limit of the liposomal structures. ${ }^{45}$ There also exists some recent reviews on remotely ultrasound-triggered release from liposomes-based ${ }_{20}$ DDS, such as those by Perrie and coll., ${ }^{26}$ Carlisle and coll. ${ }^{46}$ and Schiffelers and coll. ${ }^{47}$ While the designing and use of hybrid nanovehicles for remotely ultrasound-triggered release is discussed in the following sections.

\section{${ }_{25}$ 3. Fabrication of different types of hybrid nanovehicles}

The use of nanotechnology offers a beneficial platform to design various nanostructures for DDS, and several families of nanovehicles have been employed to either passively or actively 30 deliver chemotherapeutic payloads. As an ideal DDS, the system should be an active participant, rather than passive drug carrier, making it possible to regulate the release behaviours. ${ }^{22}$ A large number of stimuli have been reported to trigger the cargo release, and inorganic nanoparticles, which possess sensitivity to remote 35 stimuli, such as gold nanorods to NIR light, ${ }^{48}$ superparamagnetic nanoparticles to magnetic fields, ${ }^{24}$ have been widely engineered into those hybrid nanovehicles. These hybrid nanostructures are endowed with multi-functions originating from each component, ingeniously building a smart connection among those functions.

${ }_{40}$ Generally, upon those remote triggers, temperature increase or thermal energy generated within those nanovehicles is the driving force, which is exploited to induce the chemo- and/or physical responses of the thermo-responsive component, and subsequently trigger the drug release. ${ }^{33}$ Thus, by selecting the right local

45 heating generators and nanostructured assemblies, coupling with these thermo-sensitive components, one could envision a safe entrapment of toxic chemotherapeutic ingredients during the in vivo circulation and/or upon contacting with those non-targeted tissues; however, the chemotherapeutic payloads would be only

50 readily available after targeting to a site of interest and the activation of remote triggers. ${ }^{29}$ In the following context, we will classify the hybrid nanovehicles into different categories according to their structural design, even if for some specific nanostructures, they might affiliate to two or more of these 55 categories. Furthermore, some representative proof-of-concept models of the hybrid nanovehicles have been selected for further detailed discussion.

\section{1. Hybrid host/guest conjugated nanostructures}

60 Compared with other complicated strategies, one of the simplest routes to load guest molecules is to fabricate a host/guest conjugate through direct immobilization of the guest molecules onto the host nanovehicles, via covalent bonding or physicochemical interactions, e.g. electrostatic or van der Waals' 65 interactions. For GNPs aimed to DDS, some reports have been devoted to anchor sulfur-bearing DNA molecules directly onto the GNPs, e.g. gold nanospheres ${ }^{49}$ and gold nanorods, ${ }^{50-54}$ via the robust $\mathrm{Au}-\mathrm{S}$ conjugation. However, due to the high affinity between gold and sulfur atoms $(c a .126 \mathrm{~kJ} / \mathrm{mol}),{ }^{55}$ higher-power 70 NIR irradiation or higher local temperature is needed to cleave the $\mathrm{Au}-\mathrm{S}$ conjugation and subsequently trigger the release of therapeutically-active DNA modalities. Moreover, this kind of remotely triggered release is very often accompanied with shape transformation of GNPs due to the intensive local heating, and 75 sometimes accompanied with thermo-induced degradation of the chemotherapeutic payloads, resulting in a lowered therapeutic efficiency.

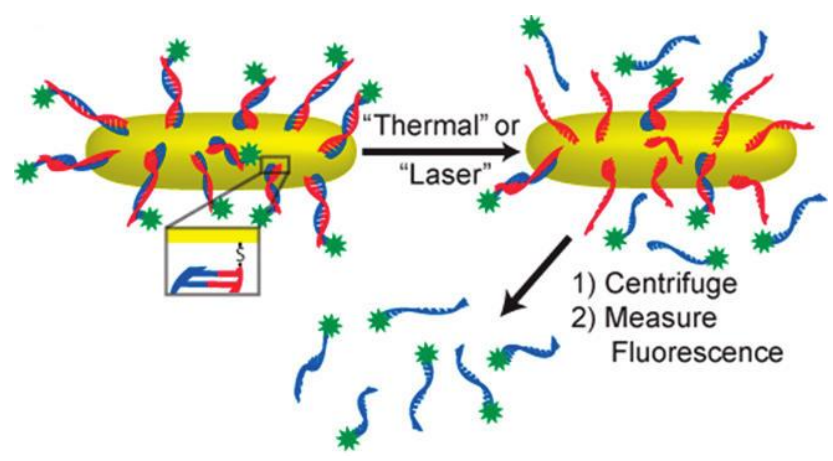

${ }_{80}$ Fig. 2 The thiolated ssDNA sequence (red) attaches to the gold surface first, then the complementary ssDNA sequence (blue) tagged with a fluorescein molecule (green) was loaded via base paring. Upon heating (thermal treatment) or illumination with laser light (laser treatment) the blue sequence is released and separated from the nanorods by 85 centrifugation. Adapted with permission from Ref. 56. Copyright 2012 American Chemical Society.

To overcome such kind of drawbacks, current researches shifted to the strategy of dehybridizing double-stranded DNA (dsDNA), in order to release a single-stranded DNA (ssDNA) via 90 mild, other than intensive, NIR irradiation. To build these hybrid nanosystems, one ssDNA chain with a thiol-end group is first immobilized, similarly to the former strategy. A therapeutically- 
active ssDNA chain is then hybridized with the former one via Watson-Crick base-pairing. Thus, the underlying mechanism for the NIR-triggered release is the dehybridization of the dsDNA chains under NIR irradiation, which requires less energy 5 (compared to the previous strategy), thus lower NIR power is needed. Based on this concept, some new GNPs/DNA conjugates were fabricated for remotely NIR-triggered release. ${ }^{56-65}$ As an illustrative example with the work of Halas and coll., ${ }^{56}$ Fig. 2 shows the engineered nanovehicles based on gold nanorods, 10 aiming for the "on-demand" NIR-triggered release of therapeutic ssDNA. Specifically, the host and cargo ssDNA chains were hybridized first, and then the dsDNA were bound to the gold nanorods surfaces via the thiol modification on the host ssDNA. At the same time, the cargo ssDNA was tagged with a fluorescein 15 molecule in order to quantify the cargo ssDNA loading and release. The DNA oligonucleotides are expected to be released when the dsDNA is dehybridized under NIR irradiation, which are detailed in the following section on variant release mechanisms. Similar DNA immobilization strategy was also 20 reported in the conjugation of dextran-coated iron oxide and DNA by Bhatia and coll. ${ }^{66}$, GNPs@ $\mathrm{CoFe}_{2} \mathrm{O}_{4} \mathrm{NP}$ and thiolated oligonucleotide by Baglioni and coll. ${ }^{67}$. Additionally, other guest molecules could also be anchored onto the surface of gold or magnetic nanoparticles via other thermo-labile covalent bonds, ${ }_{25}$ such as azo-group reported by Pellegrino and coll. ${ }^{68}$ and DielsAlder cycloaddition bonding by Niidome and coll. ${ }^{69}$

\section{2. Inorganic/organic core/corona nanostructures}

The fabrication strategy of hybrid inorganic/organic 30 core/corona nanostructures consists of the non-covalent immobilization of macromolecules chains onto the surface of inorganic nanoparticles via the "grafting to" strategy, and covalent immobilization via the "grafting from" or "grafting to" strategies. ${ }^{70}$ Through the non-covalent method, macromolecular 35 chains can be immobilized via physical interactions, e.g. Van der Waals' or electrostatic interactions, in an easy way. ${ }^{71}$ The advent of efficient controlled radical polymerization (CRP) techniques has allowed to precisely design a large variety of stimuliresponsive macromolecules, which have been used to form a 40 functional polymer corona around the inorganic cores, such as gold or magnetic nanoparticles, via the "grafting from" or "grafting to" strategy. ${ }^{72}$ Then the guest molecules could be loaded within the stimuli-responsive polymer corona, and release is subsequently triggered upon appropriate remote stimuli.

45 As reported in the work of Jérôme and coll. ${ }^{19,}{ }^{73}$, a typical non-covalent "grafting to" strategy was used to fabricate such kind of hybrid nanovehicles. Poly(acrylic acid)- $b$-poly(vinyl alcohol) (PAA- $b$-PVOH) double-hydrophilic block copolymers, successfully synthesized from CMRP technique, ${ }^{74,} 75$ were 50 anchored to the surface of 7.5-nm maghemite cores $\left(\gamma-\mathrm{Fe}_{2} \mathrm{O}_{3}\right.$ NPs) via electrostatic interaction. This inorganic/organic hybrid nanostructure was proved to concurrently possess responsive to variation in $\mathrm{pH}$, ionic strength, as well as AMF. Positivelycharged guest molecules, like methylene blue (MB), could be 55 easily loaded via electrostatic interaction with negatively-charged PAA segments at neutral $\mathrm{pH}$. On the other hand, presence of PVOH block imparts the resultant $\gamma-\mathrm{Fe}_{2} \mathrm{O}_{3} @ \mathrm{PAA}-b-\mathrm{PVOH}$ NPs with improved colloidal stability in aqueous medium, especially at $\mathrm{pH}$ below the $\mathrm{p} K_{\mathrm{a}}$ of PAA, and also capability to escape the ${ }_{60}$ MPS clearance. Aside by the $\mathrm{pH}$ and IS-triggered release, on account of the local heating capacity of $\gamma-\mathrm{Fe}_{2} \mathrm{O}_{3}$ cores, remotely AMF-induced release of MB was also evidenced in this work. Similar non-covalent "grafting to" routines have also been reported by Lecommandoux and coll., ${ }^{76}$ lanthanum strontium 65 manganese oxide nanoparticles with a silica shell was deposited with well-defined poly(lysine)- $b$-poly(ether) copolymer through electrostatic interactions. Then, DOX was loaded into the thermoresponsive poly(ether) domain via van der Waals' interaction. Release of DOX was triggered by remote AMF application, due 70 to the shrinkage of polymer corona once local temperature exceeded the lower critical solution temperature (LCST) of the poly(ether) block.

However, this non-covalent "grafting to" strategy might suffer from the drawback of labile immobilization of polymer 75 chains, which might further lead to the structural and/or colloidal instability of such hybrid nanoparticles. Moreover, limited corona thickness might also further confine the possibility of improving drug loading capacity. ${ }^{77}$ Compared to the "non-covalent" strategy, the "covalent" strategy might possess more control over 80 the surface grafting density, thus the surface properties could be more easily tuned. In most cases, this "grafting to" strategy involves macromolecules bearing a specific functional end-group, which exhibits high affinity to the inorganic matrix. Representative examples are macromolecules prepared by 85 reversible addition-fragmentation transfer (RAFT) polymerization, leading to macromolecules bearing a trithiocarbonate, dithioester, or xanthate end-groups, which are known to strongly bind to gold surface via Au-S conjugation. ${ }^{78}$ In addition, macromolecules with sulfur-bearing end-groups from 90 post-modification could also be used for this purpose. Up to now, there are some reports on forming thermo-responsive corona over GNPs with $\operatorname{poly}\left(N\right.$-isopropyl acrylamide) (PNiPAAm) ${ }^{79-83}$ $\operatorname{poly}\left(N\right.$-vinylcaprolactam) (PNVCL) ${ }^{84}$ or gold nanorods with poly(ethylene glycol)- $b$-poly $(N \text {-vinylcaprolactam })^{85}$ from RAFT ${ }_{95}$ technique. Other thiol-ended macromolecules from postfunctionalization were also reported to coat GNPs, such as the work reported by Zhong and coll. ${ }^{86,87}$ and Jérôme and coll. ${ }^{88}$ on GNRs, which were coated with thiol-ended poly( $\varepsilon$-caprolactone)$b$-poly(ethylene glycol) PCL- $b$-PEG for remotely NIR-triggered 100 release.

The "grafting from" strategy here, also called "surfaceinitiated polymerization" (SIP), ${ }^{77}$ allows to effectively immobilizing macromolecular chains onto inorganic matrices with one of the extremities tethered onto the surface. Compared 105 with the non-covalent "grafting to" technique, the "grafting from" approach enables to reach a much higher grafting density. ${ }^{89}$ The resulting hybrid inorganic/organic nanostructures are endowed with various specific features, owing to the rapid progress in CRP techniques, which are applicable to various functional monomers. 11090 Functional coating of GNPs with stimuli-responsive macromolecules via the SIP technique has been reported by Zhang and coll. ${ }^{91}$ on GNPs with dextran-based $\mathrm{pH}-$ and temperature-sensitive coating (PNiPAAm), Niidome and coll. ${ }^{79}$ on GNR@PNiPAAm, Perez-Juste and coll. ${ }^{92}$ on 115 GNP@PNiPAAm core/corona hybrid nanostructures, etc. 


\section{Cite this: DOI: 10.1039/c0xx00000x}

\section{www.rsc.org/xxxxxx}

ARTICLE TYPE

Here is a representative work of hybrid inorganic/organic nanostructures from SIP technique successfully developed by Ji and coll. ${ }^{93}$ as DDS for remotely triggered release. As shown in Fig. 3, GNRs@PNiPAAm nanostructures were prepared via the 5 surface-initiated atom transfer radical polymerization (ATRP) technique (Fig. 3a and 3b), with a final SPR band at $843 \mathrm{~nm}$. Thanks to the thermo-responsiveness arising from the PNiPAAm polymer corona, swelling/de-swelling transition around the LCST can remarkably affect hydrodynamic diameter of the ${ }_{10}$ GNRs@PNiPAAm nanohybrids, the surface refractive index and
SPR band (Fig. 3c), as well as transmission of the suspension (Fig. 3d). Additionally, short bursts of NIR irradiation $(\lambda=808$ $\mathrm{nm}, 1.5 \mathrm{~W}$ ) heated the particles to temperature above their LCST, causing the hydrodynamic diameter of the nanoparticles shifting 15 from below $100 \mathrm{~nm}$ to $c a .600 \mathrm{~nm}$, due to the thermo-induced phase-change. The thermo-responsive polymer shells allowed loading norvancomycin molecules, a widely-used glycopeptide antibiotic against methicillin-resistant Staphylococcus aureus, via hydrogen bonding. A controlled and pulsatile release of 20 norvancomycin was also demonstrated in this work.
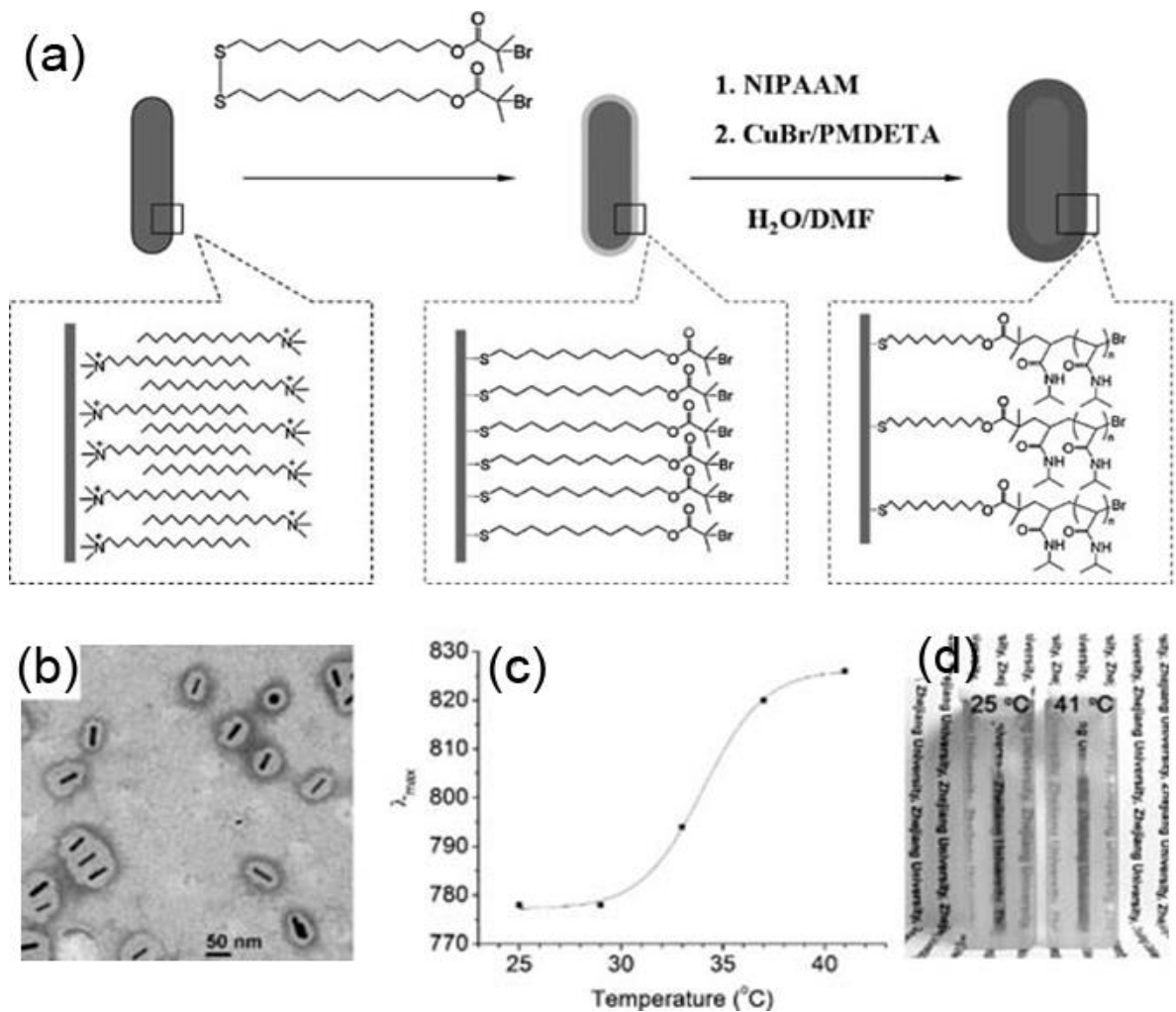

Fig. 3 Preparation of GNR/PNiPAAm hybrid nanostructures via surface-initiated ATRP (a), representative TEM image of GNR/PNiPAAm nanohybrids (b), the fitted plotting of longitudinal surface plasmon wavelengths of GNR/PNiPAAm vs. temperature (c), and digital photographs of GNR/PNiPAAm 25 solutions at 25 and $41^{\circ} \mathrm{C}$, respectively. Adapted with permission from Ref. 93. Copyright 2008 WILEY-VCH Verlag GmbH \& Co. KGaA, Weinheim.

\section{3. Hybrid polymeric nanogels}

Smart nanogels, which are nano-sized physically- or chemically-cross-linked networks made of hydrophilic and 30 functional macromolecules, have been considered for various biological applications, due to their capacity to hold a large amount of water or biological fluids in the swollen state. Furthermore, they also undergo physicochemical changes responding to external stimuli, such as variation in $\mathrm{pH}$ or 35 temperature, activation of biological molecules, like glucose and enzyme, etc. ${ }^{94}$ The stimuli-induced conformational changes of these nanogels affect significantly the release behaviours. For instance, thermo-induced collapsing of the thermo-responsive nanogels lead to a burst release, when local temperature 40 overreaches the LCST. ${ }^{85,95}$ That is why magnetic and gold nanoparticles integrated with biocompatible nanogels hold great promise as DDS, and have been increasingly studied. Generally, the most commonly used method to fabricate these hybrid nanogels is the SIP strategy, which has been discussed in detail in 45 the former section. Some works have been reported on gold $^{79,96}$, 97 and magnetic ${ }^{98-100}$ nanoparticles-based nanogels, to cite a few, and representative example could also be referred to the work of 
Ji and coll., ${ }^{93}$ as shown in Fig. 3. In this section, strategies to fabricate the hybrid polymeric nanogels, other than SIP, are detailed.

A typical chemically cross-linked hybrid nanogels from 5 polymerization is depicted in the work of Zhou and coll., ${ }^{101}$ through the free radical precipitation copolymerization of NiPAAm, AAm and fluorescent carbon dots embedded in the porous carbon shell and magnetic iron oxide nanocrystals clustered in the core, using APS as initiator. The reversible 10 swelling and shrinking of the poly(NiPAAm-co-AAm) shell in response to temperature changes not only modify the physicochemical environment of the embedded bifunctional nanoparticles (fluorescent carbon dots embedded in the porous carbon shell and superparamagnetic iron oxide nanocrystals 15 clustered in the core) for sensing on the local environment, but also change the mesh size of the gel networks to regulate the drug release. A more recent work reported by Hayashi and coll. ${ }^{102}$ dealt with the in situ synthesis of hybrid nanogels. First, they polymerized pyrrole-3-carboxylic acid (PyCOOH) using iron (III) 20 ion as a catalyst in an aqueous solution of DOX and PVA to synthesize DOX-containing carboxylic polypyrrole (DOX/PPyCOOH) nanogels. In this process, DOX was introduced within the NPs via the $\pi-\pi$ interaction between $\mathrm{PPyCOOH}$ and DOX. In addition, the carboxylic acid groups of 25 the DOX/PPyCOOH nanogels were used later for surface modification. Second, they synthesized $\mathrm{Fe}_{3} \mathrm{O}_{4}$ NPs within the $\mathrm{DOX} / \mathrm{PPyCOOH}$ nanogels by reducing part of the used iron (III) ions to iron (II) ions with hydrazine. Finally, to increase the NP retention in the tumour, the nanogels were modified with PEG 30 and folic acid via the amidation reaction. This protocol leads to hybrid nanogels, with average size of $c a .70 \mathrm{~nm}$, with promising remotely-AMF-triggered release.

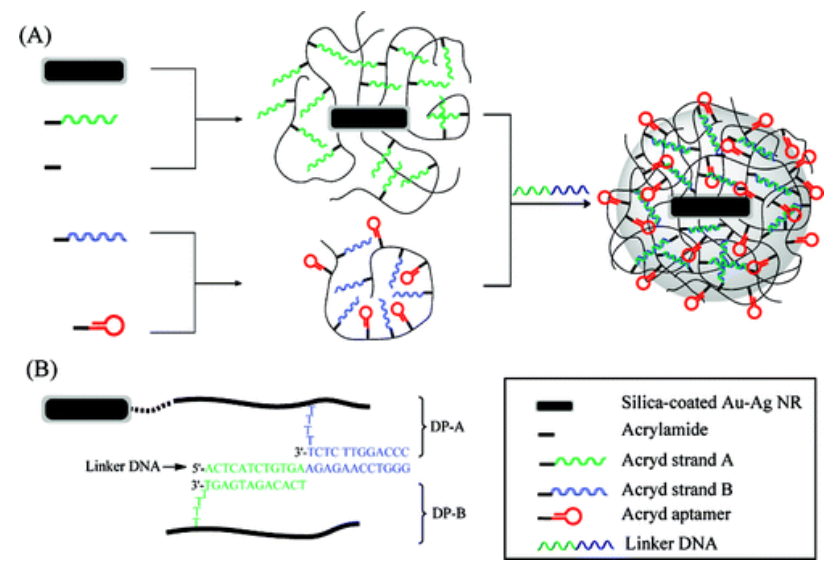

Fig. 4 Schematic diagram illustrating the formation of an aptamer35 functionalized core-shell nanogel (A). DNA sequences and linkages in the nanogels (not to scale, B). Adapted with permission from Ref. 103. Copyright 2011 American Chemical Society. (a)
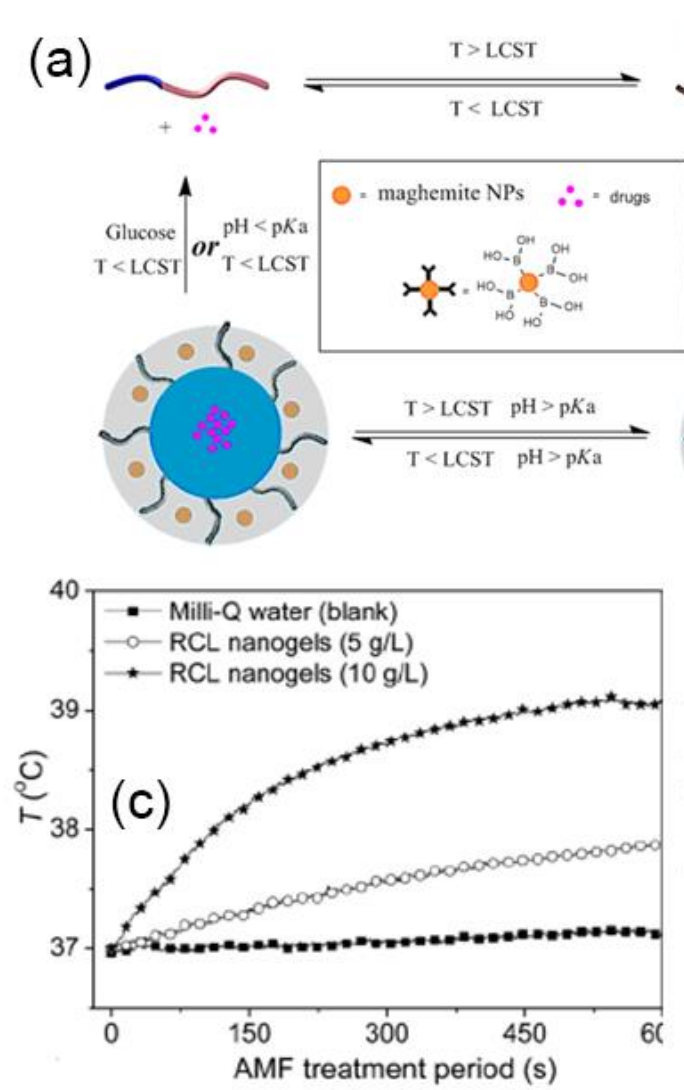
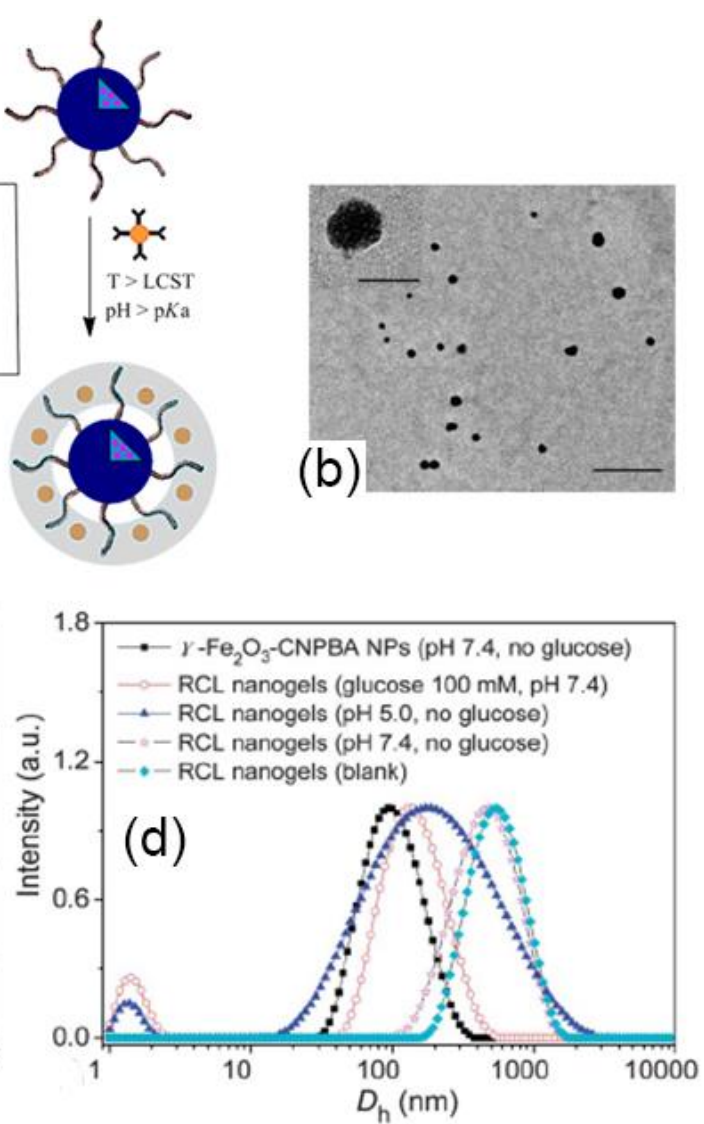

40 Fig. 5 Preparation of reversibly cross-linking (RCL) nanogels from PVOH- $b$-PNVCL with surface-functionalized $\gamma$-Fe ${ }_{2} \mathrm{O}_{3}$ NPs via the boronate/diols bonding and loading of hydrophobic cargoes (a); representative TEM image of the RCL nanogels (scale bar: 500 nm), and inset: partially-magnified TEM image of a nanogel particle (scale bar: $100 \mathrm{~nm}$ ) (b); AFM-induced heating profiles of the RCL nanogel aqueous solutions (10 and $5 \mathrm{~g} \mathrm{~L}^{-1}$ ) under 


\title{
Cite this: DOI: $10.1039 / \mathrm{c0xx00000x}$
}

\author{
www.rsc.org/xxxxxx
}

ARTICLE TYPE

alternating magnetic field (AMF, $755 \mathrm{kHz}, 14 \mathrm{mT})(\mathrm{c})$; size distribution of the RCL nanogels after $6 \mathrm{~h}$ treatment $\left(37{ }^{\circ} \mathrm{C}\right)$ of glucose $(100 \mathrm{mM}$, pH 7.4$)$, acidic $\mathrm{pH}$ ( $\mathrm{pH} 5.0$, no glucose) and physiological $\mathrm{pH}$ (pH 7.4, no glucose) (d). Adapted from Ref. 104 with permission from The Royal Society of Chemistry.

In light of the limited control over the release due to non5 reversible cross-linking, reversible cross-linking was recently introduced in order to impart a better regulation over the release behaviours. ${ }^{28}$ Tan and coll. ${ }^{103}$ reported cross-linked hybrid nanogels based on $\mathrm{Au}-\mathrm{Ag}$ nanorods (Au-Ag NRs) and DNA cross-linked polymer shells as new DDS. As shown in Fig. 4, 10 anticancer drugs were encapsulated into the polyacrylamidebased polymer shell, and DNA complementarities were applied to form the cross-linked structures. Moreover, the Au-Ag NRbased nanogels can also be readily functionalized with targeting moieties of aptamers, with specific recognition of tumour cells. 15 When exposed to NIR irradiation $(808 \mathrm{~nm}, 600 \mathrm{~mW})$, the photothermal effect generated by $\mathrm{Au}-\mathrm{Ag} \mathrm{NRs}$ led to a fast decross-linking of the formerly cross-linked nanostructures, followed with the release of the encapsulated payload with high control.

20 In the report of Jérôme and coll., ${ }^{104}$ reversibly cross-linked thermo-responsive nanogels based on poly(vinyl alcohol)- $b$ poly $(N$-vinylcaprolactam) copolymers and maghemite nanoparticles ( $\gamma-\mathrm{Fe}_{2} \mathrm{O}_{3} \mathrm{NPs}$ ) were developed. As shown in Fig. 5, thermo-responsive PVOH- $b$-PNVCL copolymer from the CMRP 25 technique $^{74,105,106}$ formed self-assembled micelles above the LCST in aqueous medium. Then, 7.5-nm $\gamma-\mathrm{Fe}_{2} \mathrm{O}_{3} \quad \mathrm{NPs}$ functionalized with boronic acid moieties were used to reversibly crosslink the PVOH corona via the boronate/diols bonding. Then thermo-, glucose- and pH-responsive reversibly cross-linked 30 nanogels were obtained after cooling down below the LCST (Fig. 5a and 5b). The as-prepared RCL nanogels showed a good responsiveness to the presence of glucose and/or acidic pH (Fig. 5d) due to the boronate/diol complex. Moreover, presence of $\gamma$ $\mathrm{Fe}_{2} \mathrm{O}_{3}$ NPs also imparted the RCL nanogels with capability to 35 generate local heating under AMF application (Fig. 5c). Therefore, after loading with a hydrophobic drug within the PNVCL domain, $\mathrm{pH}$ - or glucose-triggered drug release could be accomplished. Furthermore, magnetic heating capacity, originating from the maghemite components, also allowed the 40 triggered release under remote AMF application.

\section{4. Hybrid polymer micelle nanostructures}

Polymer micelles, obtained by the intermolecular packing of amphiphilic copolymers, are serving as powerful tools in 45 biomedical applications, such as DDS and nano-bioreactors, by virtue of their outstanding advantages in drug loading capacity and also cellular uptake efficiency. ${ }^{1,3}$ Lower critical micellar concentration of polymer micelles imparts them with relatively superior stability in aqueous solution, compared to conventional 50 micelles like liposomes. On the other hand, their homogeneous size, hydrophilic corona and nano-scaled dimension can effectively hinder the fast exclusion by renal clearance and MPS processes, and also they could accumulate within the tumour sites via the well-established EPR effect. ${ }^{23}$ Additionally, the 55 hydrophobic reservoir can accommodate different ingredients, e.g. biomedical imaging agents, toxic chemotherapeutics, or both. Once stimuli-responsive blocks are introduced, release of the pre-loaded drug payloads can be triggered by external stimuli such as light, redox agents, variation in $\mathrm{pH}$, temperature, ionic 60 strength, etc. ${ }^{6}$ Moreover, after integration of inorganic components, e.g. magnetic or gold nanoparticles, multiple biomedical functions are foreseen, such as medical diagnosis, tumour ablation and remotely triggered release, etc..$^{48,107,108}$

Generally, inorganic nanoparticles, such as iron oxide and 65 gold nanoparticles, are stabilized with a surfactant before use, such as oleic acid, citrate salt, or cetyltrimethylammonium bromide, etc. Due to the toxic essence of most surfactants, surface modification becomes mandatory before in vivo use. The most commonly-used strategies consist of ligand exchanging, 70 masking with other biocompatible and hydrophilic surfactants, encapsulating within other vehicles, etc. ${ }^{37}$ These methods are supposed to endow them with superior stability in aqueous medium or biological fluid, and/or to improve the biocompatibility of the system. As a key prerequisite to engineer 75 this kind of hybrid polymer micelles for remotely-triggered release, a high loading level of both inorganic nanoparticles and chemotherapies is mandatory. The encapsulation strategy might effectively satisfy this requirement, by manipulating the coassembly of amphiphilic copolymers with hydrophobic cargoes 80 into micelle nanostructures. Polymer micelles, encapsulating chemotherapeutic agents together with magnetic nanoparticles, 109-111 gold nanoparticles, ${ }^{86,112-114}$ or upconverting nanoparticles (UCNPs, NaYF4:TmYb), ${ }^{115-118}$ hold great promises for remotelytriggered release. Some of them have already been successfully ${ }_{85}$ proved, such as NIR-triggered release from polymer micelles. ${ }^{86}$, 116

Nevertheless, because of their dynamic (non-cross-linked) nature, those polymer micelles may encounter some critical challenges, e.g. unexpected dissociation and subsequent 90 premature release, due to the dynamic equilibrium with the free polymer chains. Moreover, dilution of self-assembled polymer micelles upon in vivo intravenous administration might also lead to the same deficiency. ${ }^{119}$ Fortunately, micelle stability can be significantly improved via reversible or non-reversible covalent 95 cross-linking. ${ }^{119-121}$ We will not detail more on these crosslinking strategies here, but only highlight the cross-linking rational in designing hybrid polymeric micelles for remotely triggered release.

A representative example has been reported by Chen and 100 coll., ${ }^{122}$ dedicated to the cross-linked hybrid micelles, with 
encapsulated magnetic nanoparticles and chemotherapeutic agents (Fig. 6). Specifically, amphiphilic surfactant of PEOPPO-PEO readily forms oil-in-water micelles with a PPO core and PEO corona, while $\mathrm{Fe}$ salts were present within the inner 5 water phase. 4-Nitrophenyl chloroformate, gelatin and 1-ethyl-3(3-dimethylaminopropyl) carbodiimide were added to cross-link the micelle nanostructures. At the same time, the Fe salts were precipitated into magnetic nanoparticles via alkaline hydrolysis, thus the magnetic nanoparticles were incorporated within the 10 cross-linked nanostructures. The cross-linking was confirmed to impart a good stability to the colloids without substantially affecting the thermo-responsiveness of the copolymer. These spherical nanostructures exhibited a hydrophilic/hydrophobic transition around $40{ }^{\circ} \mathrm{C}$, accompanied with a size contraction and 15 shrinkage of the core. The core content, experiencing very slight leakage at $25{ }^{\circ} \mathrm{C}$, possessed a half-life about $5 \mathrm{~h}$ at $45{ }^{\circ} \mathrm{C}$, but burst out within a few minutes under magnetic heating due to the iron oxide coarsening and core/shell disruption. Such burst-like response facilitates the release of Vitamin B12, under remote 20 AMF activation.

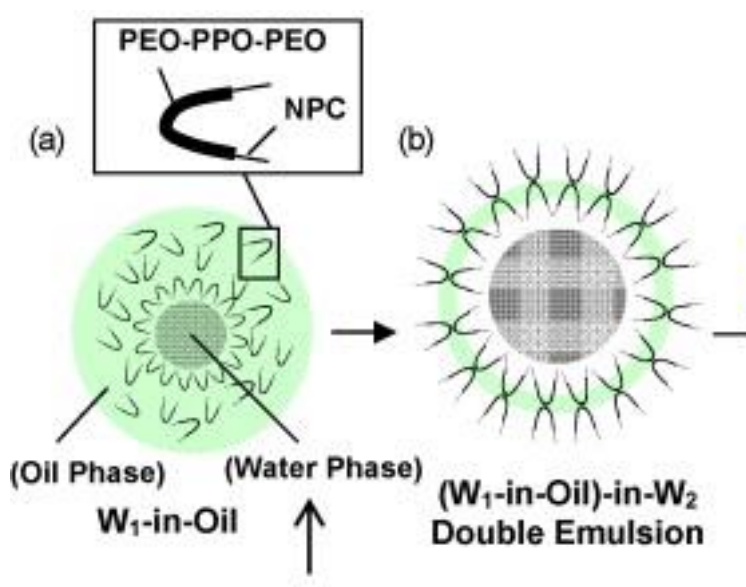

Drug and iron oxide precursors

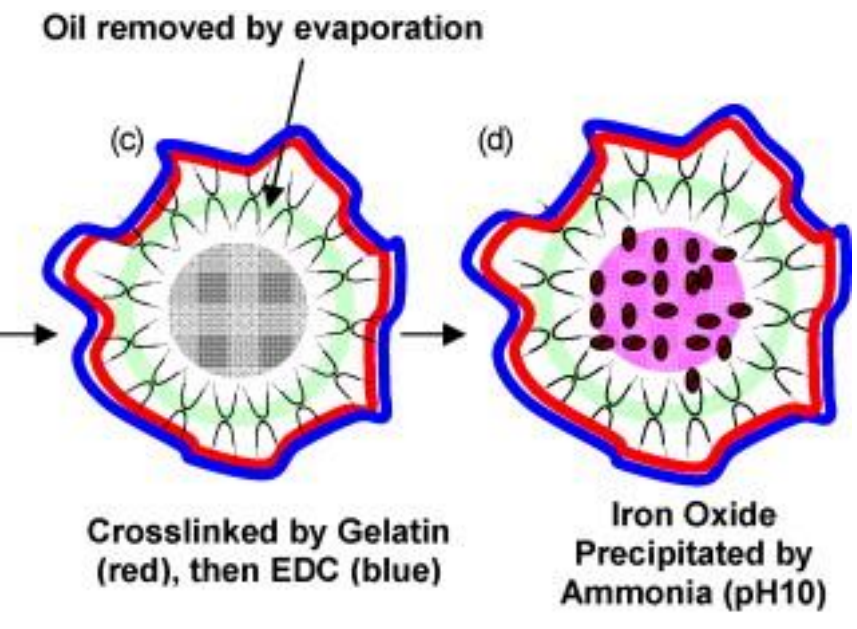

Fig. 6 A self-assembly strategy of aqueous nanovehicles using double emulsion for drug delivery under combined magnetic and thermal stimuli. "W": a water phase made of PBS/hydrophilic drug/Fe salts mixture, "W ${ }_{2}$ ": PBS buffer, "Oil": PEO-PPO-PEO/ $\mathrm{CH}_{2} \mathrm{Cl}_{2}$ solution. The PEO-PPO-PEO was end-

25 functionalized with 4-nitrophenylchloroformate (NPC) for subsequent cross-linking with gelatin. (a) Adding $\mathrm{W}_{1}$ to oil phase to form an inverse micelle emulsion; (b) adding this emulsion to $\mathrm{W}_{2}$ to form micelle dispersion with a PEO-PPO-PEO shell; (c) the PEO shell was cross-linked with gelatin at $4^{\circ} \mathrm{C}$ with 1-ethyl-3-(3-dimethylaminopropyl)- carbodiimide (EDC) as catalyst; meanwhile, $\mathrm{CH}_{2} \mathrm{Cl}_{2}$ residue can be removed by evaporation; (d) iron oxide nanoparticles can be precipitated by adding ammonia to raise $\mathrm{pH}$ to 10 under modest heating of $60{ }^{\circ} \mathrm{C}$. Adapted from Ref. 14, copyright 2009 Elsevier.

\section{3. 5. Hybrid liposome nanostructures}

Phospholipids are molecules that bear both a hydrophobic (non-polar tail) and a hydrophilic (polar head) component. In aqueous solution, these amphiphilic molecules spontaneously self-assemble into nano-sized liposomes, in which a single and 35 continuous bilayer of phospholipids segregates the internal aqueous cavities from external surroundings. ${ }^{123}$ This specific structuration makes liposomes appealing as reservoirs for molecules of interest. Similarly to the polymer micelle, lots of researches on liposomes have also been reported in the field of 40 DDS. As reported by Mulder and coll., ${ }^{124}$ sizes and geometries of the liposomes can be manipulated by tuning the essence or/and tail length of each part of the phospholipids, as shown in Fig. 7. Moreover, other parameters, e.g. concentration, temperature and $\mathrm{pH}$, could also lead to different aggregates in aqueous medium. It 45 is deserved to be noted that, advances in the liposome research have led to the development of liposomes composed of lipids with a hydrophilic counterpart of PEG, also known as "stealth liposomes". This specific nanostructure allows a prolonged circulation period, passive accumulation in tumour tissues via the ${ }_{50}$ EPR effect. ${ }^{27}$ Generally, hydrophilic drugs are typically loaded into liposomes by passive entrapment during the liposome formation. Weakly-alkaline amphiphilic drugs could also be encapsulated with the help of transmembrane gradients or trapping agents to form insoluble complex, leading to a higher 55 loading efficiency. More details on the loading of guest molecules within the liposome-based vehicles have already been referred thoroughly in the review of Drummond and coll. ${ }^{125}$

The concept of stimuli-triggered release was further extended since the report on thermo-sensitive liposomes (TSLs) by Yatvin 60 and coll. ${ }^{126}$ and Weinstein and coll. ${ }^{127}$ TSLs can release the encapsulated cargoes near their melting temperature $\left(T_{\mathrm{m}}\right)$, where the lipid membrane undergoes a phase transition from gel to liquid crystalline state. Thermo-sensitive phospholipids, e.g. dipalmitoyl glycerophosphocholine (DPPC, $T_{\mathrm{m}}$ of $41{ }^{\circ} \mathrm{C}$ ) and ${ }_{65}$ distearoyl glycerophosphocholine (DSPC, $T_{\mathrm{m}}$ of $55{ }^{\circ} \mathrm{C}$ ), have been used to fabricate DDS for thermo-triggered release. ${ }^{126,127}$ In those TSLs-based DDS, chemotherapeutic molecules were encapsulated, and extravasation of the cargoes into healthy tissue was highly quenched at physiological temperature. However, ${ }_{70}$ TSLs were able to release the encapsulated drugs at or above $T_{\mathrm{m}}$ of the lipid bilayers. Thus, mild heating of a tissue could induce the local delivery of chemotherapeutics within the disease sites. ${ }^{128,129}$ Even though there have been some practices to trigger the release with conventional hyperthermia through heating, 75 clinical translation of temperature-triggered drug delivery might be still hampered, in light of the challenge to establish and 


\section{Cite this: DOI: 10.1039/c0xx00000x}

\section{www.rsc.org/xxxxxx}

ARTICLE TYPE

maintain a local hyperthermia, leading to uncontrollable release behaviours.

An alternative approach involves the application of ultrasound or encapsulation of inorganic heating generators, such 5 as iron oxide or gold nanoparticles. The combination of TSLs and ultrasound for remotely triggered release involves the ultrasound hyperthermia and/or ultrasound-induced cavitations, ${ }^{130}$ which have been referred in Section 2.3. However, the introduction and role of inorganic heating generators are going to be highlighted 10 here. Similarly to the hybrid polymer micelles, inorganic nanoparticles could be also easily encapsulated within the liposomes. The formulation and use of multi-functional and multi-component formulated liposomal nanoparticles, sometimes referred as "theranostics", are being extensively explored. In 15 those practices, additional functions are also integrated within an individual liposome nanoparticle, such as site-specific targeting, biomarker, imaging capability, delivery of therapeutics, and responsiveness to external or internal stimuli for controlled release, etc. ${ }^{131}$ Recent advance in nanohybrid liposomes, aiming 20 for biomedical applications, includes the encapsulation of magnetic nanoparticles, ${ }^{19,}$ 124, 129, 132-141 gold nanorods, ${ }^{142,}{ }^{143}$ gold nanospheres, ${ }^{144-149}$ and gold nanoshells, ${ }^{150,151}$ to cite a few. Some proof-of-concept studies have been performed for triggered drug release via remote stimuli, such as AMF, ${ }^{129,132,137-139} \mathrm{NIR},{ }^{142-145}$, ${ }_{25}{ }^{150-153}$ and ultrasound. ${ }^{132,} 134$ It deserves to note that, as the complexity of liposome nanoparticles increases, so do the cost and difficulty associated with their manufacture and also the quality control. Therefore, a compromise between the gains in therapeutic benefits and additional expenses is of great 30 importance before the designing and fabrication.
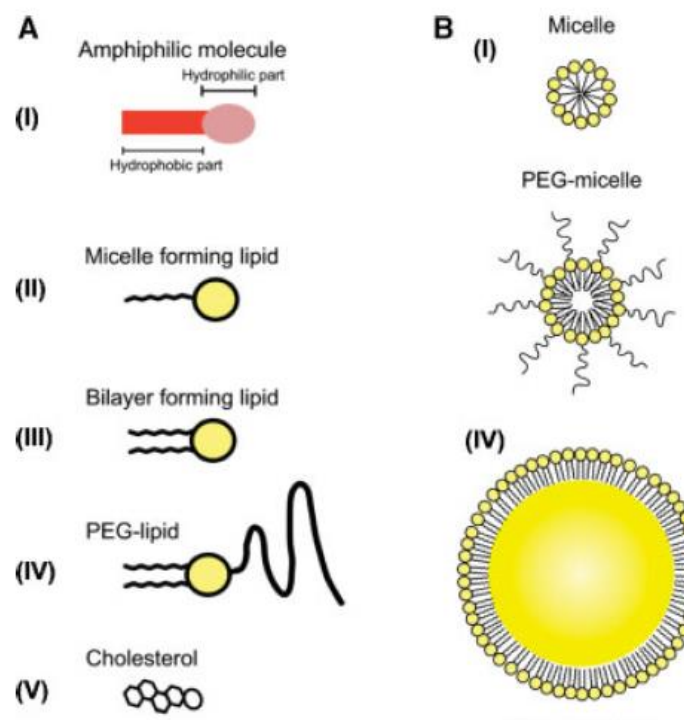

(II)
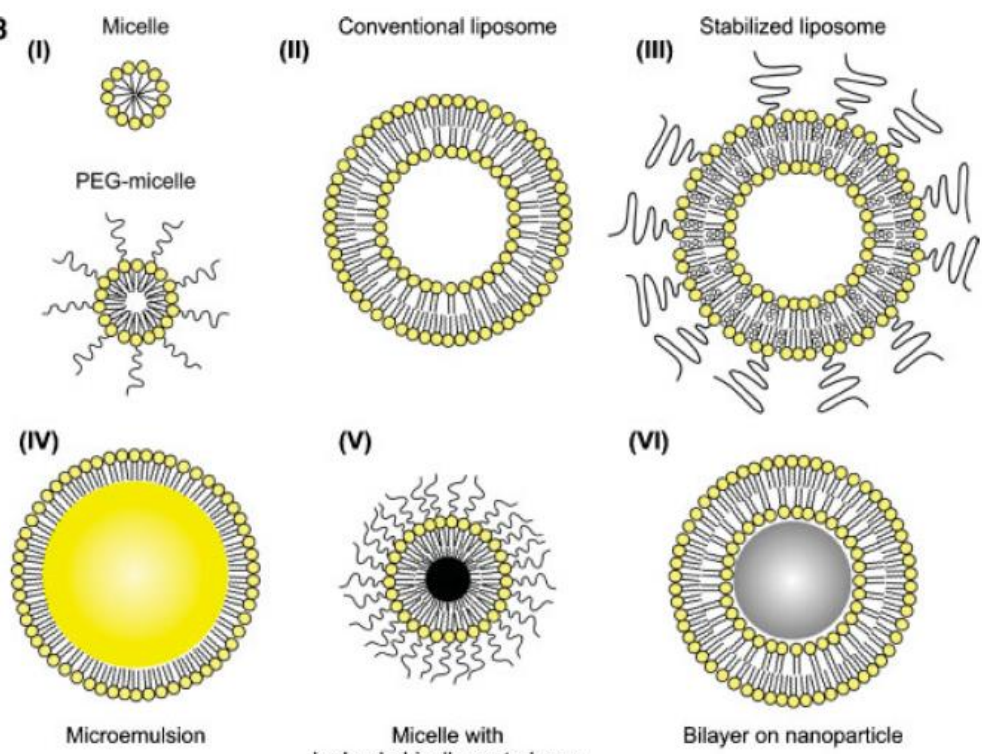

Fig. 7 (A) Schematic representation of molecular ingredients of (B) possible lipid aggregate nanostructures for in vivo use: (I) micelle from micelleforming lipids and/or from PEG-lipids; (II) conventional liposome consisting of a phospholipid bilayer; (III) stabilized liposome after incorporating a 35 small amount of PEG-lipids and/or cholesterol; (IV) microemulsion consisting of a surfactant (amphiphile) monolayer encapsulation oil; (V) micelle can containing hydrophobic nanoparticles and (VI) a lipid bilayer over nanoparticulate cores. Adapted with permission from Ref. 124 . Copyright 2006 WILEY-VCH Verlag GmbH \& Co. KGaA, Weinheim.

\section{6. Hybrid mesoporous silica-based core/shell 40 nanostructures}

Mesoporous silica nanoparticles (MSNs) show very interesting properties, e.g. stable mesoporous nanostructure, high surface areas, large pore volume, regular and tuneable mesopore size, homogeneous pore distribution, and high loading capacity 45 (up to $50 \mathrm{wt} . \%$ ) making them ideal to host guest molecules. ${ }^{154,155}$ Biocompatibility of MSNs, particularly good hemocompatibility as demonstrated by Lin and coll., ${ }^{156}$ imparts them with potency for intravenous administration. Up to now, lots of attempts have been devoted to loading drug molecules within the mesoporous 50 cavities. ${ }^{155}$ However, due to the weak interaction between the guest molecules and the inner mesoporous cavities, unavoidable premature release is usually detected, impeding their further in vivo applications. ${ }^{2}$ That is why widespread research interest has been shifted to engineering the mesoporous structures with some 55 switchable gating components, in order to open or close the cavities "on will", providing advantageous contribution to the controlled release behaviours. ${ }^{157}$ Additionally, another attractive 
feature of these capping systems should be the character of "premature zero release", when delivering those entrapped cargoes exclusively to the desirable sites. ${ }^{157,}{ }^{158}$ As far as we know, nanoparticles, phase changed materials (PCMs) and 5 supramolecular assemblies have already been attempted as gating functionality in the MSNs-based DDS, while triggered release was activated and regulated by various physical/chemical stimuli, such as reductive agent, enzyme, $\mathrm{pH}$, glucose, etc. ${ }^{159}$ This kind of MSNs-based materials, engineered with capping components and 10 possessing responsiveness to specific stimuli, have been recently reviewed in details by Zink and coll. ${ }^{159,160}$

In comparison to the chemical stimuli as mentioned above, remote physical stimuli, such as light, magnetic field and ultrasound, bear the advantage of activating the release with 15 spatial and temporal precision. In the occasion of intravenous administration, remote and non-invasive physical stimuli are preferable to control the therapeutic treatment. ${ }^{11,20,161}$ To impart them with specific sensitivity to those remote stimuli, inorganic components, e.g. gold or magnetic nanoparticles, have been 20 successfully integrated with the MSNs. Well-defined core@shell nanostructures, such as GNRs@MSNs, ${ }^{162-167}$ upconverting nanoparticles UCNP@MSNs, ${ }^{168-170} \quad \gamma$-Fe ${ }_{2} \mathrm{O}_{3} @ \mathrm{MSNs},{ }^{171}$ $\mathrm{Fe}_{3} \mathrm{O}_{4} @ M S N s,{ }^{172}, \quad 173 \quad$ MSNs@liposomes, ${ }^{174}$ and $\mathrm{Fe}_{3} \mathrm{O}_{4} @ \mathrm{Zn} @ \mathrm{MSNs}^{175}$ have been developed. Furthermore, MSNs 25 with the mesopores capped with magnetic nanoparticles, ${ }^{176-178}$ GNPs, ${ }^{179}$ UCNPs ${ }^{180}$ and sulfonatocalix[4]arene, ${ }^{167}$ oligonucleotide, ${ }^{173}$ etc., have also been used to regulate the release behaviours with remote stimuli. More recently, Kitagawa and coll. ${ }^{181}$ reported the fabrication of porous 30 aluminum coated with gold nanorods, and developed this nanostructure for NIR-triggered release of anthracene.

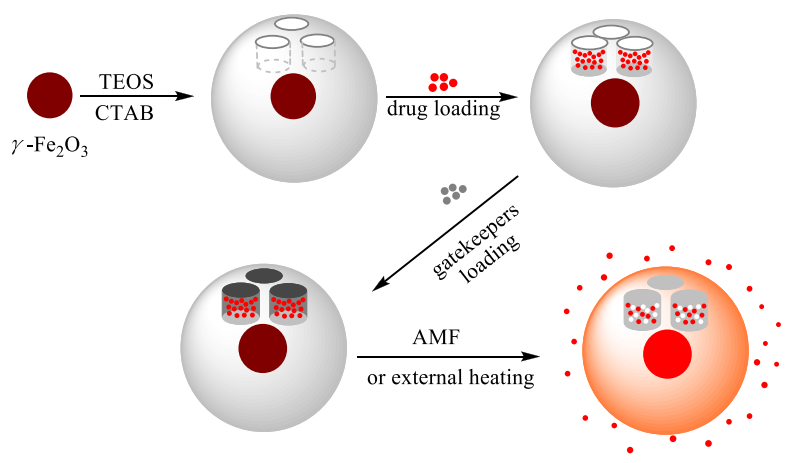

${ }_{35}$ Fig. 8 Preparation of $\gamma-\mathrm{Fe}_{2} \mathrm{O}_{3} @ \mathrm{MSNs}$, drug loading of Rhodamine ${ }^{\circledR} \mathrm{B}$ or DOX, immobilization with dodecanoic acid or 1-tetradecanol as gatekeepers, and triggered drug release by conventional external heating or AMF-induced internal heating. Adapted from Ref. 182 with permission from The Royal Society of Chemistry.

40

One representative strategy, reported by Duguet and coll., ${ }^{182}$ is the development of core-shell nanoparticles made of a $\gamma-\mathrm{Fe}_{2} \mathrm{O}_{3}$ core and mesoporous silica shell ( $\left.\gamma-\mathrm{Fe}_{2} \mathrm{O}_{3} @ \mathrm{MSNs}\right)$ as DDS (Fig. 8). Phase-changed molecules, e.g. dodecanoic acid (DA) and 145 tetradecanol (TD) with melting temperature of $45^{\circ} \mathrm{C}$ and $39{ }^{\circ} \mathrm{C}$, respectively, were introduced as gatekeepers to regulate the drug release behaviours. The release mechanism is based on the reversible thermo-induced phase change of the PCMs. Thus the pre-loaded drug molecules diffuse across the PCMs fluid (above ${ }_{50} T_{\mathrm{m}}$ ) into the release medium. On the other side, under biological condition (below $T_{\mathrm{m}}$ ), the drug molecules would be safely trapped within the cavities with the aid of those PCMs in solid state. With the $\gamma-\mathrm{Fe}_{2} \mathrm{O}_{3}$ core as heating mediator, release of cargoes is supposed to be activated via remote AMF application. In another 55 report by Duguet and coll., ${ }^{183}$ similar strategy by combining GNR@MSNs and PCMs was also used to fabricate a lightsensitive DDS, with potential for NIR-triggered release.

\section{7. Hybrid hollow nanostructures}

60 Nanoparticles with hollow interiors are increasingly more attractive in both fundamental research and practical applications, because of their unique inner void nanostructure and rigid shells providing both chemical and mechanical shielding from the surrounding environment. ${ }^{184}$ All these advantages allow efficient ${ }_{65}$ delivery of various guest molecules. In most cases, those hollow nanostructures could be prepared with those organic or inorganic components. For those with organic origins, they consist of polymer vehicles, liposomes, etc, which have already been mentioned in the former sections and aren't discussed here. In

70 this section, we mainly focus on the hollow nanostructures based on inorganics, such as silica, gold, etc.

Hollow silica nanoshells, other than mesoporous silica mentioned in the former Section 3.6, possess both a rigid mesoporous shell and interior hollow cavity. These 75 nanostructures possess a low density, high specific area and extraordinary higher cavity volume, in favour of drug loading and controlled release. ${ }^{185,186}$ Generally, hollow silica nanoshells are fabricated via a dual-template strategy, in which a hard or soft template is used to form the interior cavity, while soft template to 80 generate the mesopores in the shells. ${ }^{187}$ In order to explore the possibility to trigger the release under remote stimuli, the hollow silica nanoshells were engineered with different components, such as introducing a further gold nanoshell over the silica nanoshell, ${ }^{188-190}$ introducing gold cores $^{191}$ or magnetic cores ${ }^{192}$

85 within the nanoshells, etc. A representative work of these silicabased hollow nanostructures designed for DDS was reported by Chen and coll. ${ }^{192}$ As shown in Fig. 9, the magnetic nanoparticles and hydrophobic drugs were dissolved in oil drops and stabilized with Pluronics ${ }^{\circledR}$ (PEO-PPO-PEO) and poly(vinyl alcohol) 90 (PVA). Then the nanocomposites were coated with an ultra-thin mesoporous $\mathrm{SiO}_{2}$ shell leading to a nanoshell structure. This nanostructure underwent significant size shrinkage and the diameter decreased by more than 10 times under AMF treatment, due to the thermo-induced hydrophilic/hydrophobic transition of 95 the inner polymeric components. During this transition, it was also accompanied with solid shells destruction; therefore, remotely AMF-triggered release was achieved.

The gold-based hollow nanostructures designed for DDS usually consist of gold nanocages and nanoshells. Gold 00 nanocages, developed by Xia and coll., ${ }^{193}$ have been proposed to many applications in the biomedical field. However, the multiple pores that connects the hollow centre and the external environment, leads to the loss of all of the contents within the nanocages, if used as DDS directly. Thus, chemical or physical 105 modification over the external shell or internal cavity becomes 


\title{
Journal of \\ Materials Chemistry B
}

Cite this: DOI: 10.1039/c0xx00000x

PAPER

\author{
www.rsc.org/xxxxxx
}
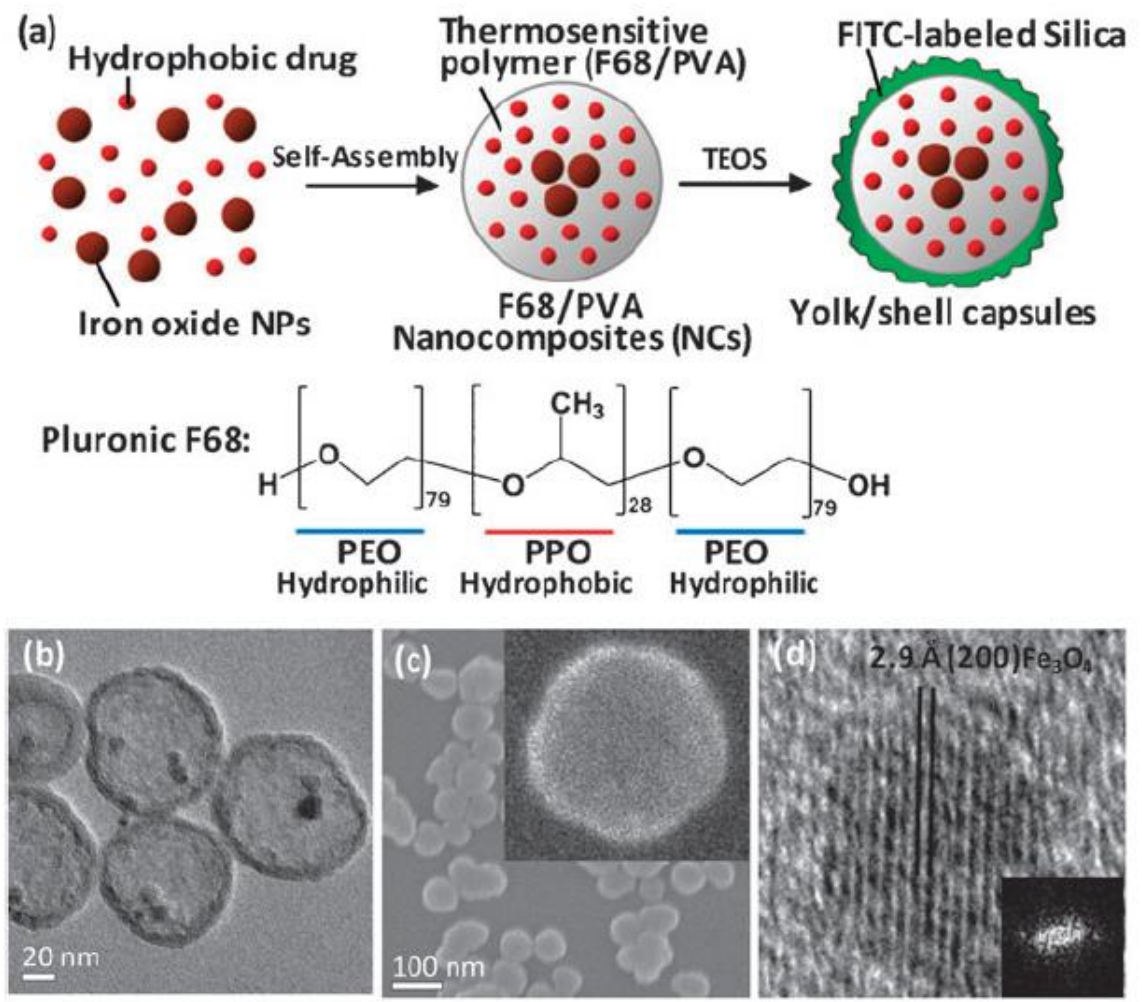

Fig. 9 Schematic illustration of the synthesis and structure of the hollow silica nanostructure for magnetically-triggered controlled drug release. The first step involved mixing the iron oxide nanoparticles and the hydrophobic drug in an organic solvent to form a uniform phase. Next, the mini-emulsion method was applied using blended polymers, poly(vinyl alcohol) (PVA) and Pluronic ${ }^{\circledR}$ F68 (PEO-PPO-PEO) as a binder to fabricate the self-assembling 5 nanocomposites. Upon the evaporation of the organic solvent, the polymers encapsulated the iron oxide nanoparticles and hydrophobic drug within the composite. Finally, the nanocomposites were coated with a thin layer of silica by hydrolysis and condensation of TEOS to obtain the final yolk/shell capsules (a). This nanostructure possesses an average diameter of about $76 \mathrm{~nm}$ and 7-nm ultra-thin silica shell (b, c and d). Adapted from Ref. $192 \mathrm{mith}$ permission from The Royal Society of Chemistry.

10 mandatory. Up to now, some attempts have been reported to coat these hollow nanostructures (gold nanocages) with PNIPAAmbased thermo-responsive copolymers ${ }^{194,} 195$ or bioactive hyaluronic ${ }^{196}$, cap the pores with immunoglobulin $\mathrm{G}(\mathrm{IgG})$ via reversible boronate/diols bonding, ${ }^{197}$ co-load guest molecules 15 together with PCMs, ${ }^{198,} 199$ Thanks to the introduction of gating components, good entrapment of cargo molecules and remotely NIR-triggered release were reported in these studies.

In addition to gold nanocages, gold nanoshells, consisting of only a thin gold wall with a hollow interior, have the unique 20 combination of small size, spherical shape and hollow interior. Moreover, a strong and tuneable absorption band in the NIR region leads their possibility to generate local heating under NIR irradiation. $^{200,} 201$ Similarly to the fabrication of hollow silica, a hard or soft template is also needed to form the interior cavity. In 25 addition to those gold nanoshells formed over the silica nanocores as mentioned above, ${ }^{188-190,202,} 203$ gold nanoshells formed over liposomes $145,148,151,204-207$ or surfactants such as cholesterylsuccinyl silane, ${ }^{208}$ and also gold nanoshells via the galvanic strategy, ${ }^{201}$ have been successfully obtained. Some of 30 these nanostructures have already been reported as DDS for remotely NIR-triggered release. ${ }^{51,} 65, \quad 150,204-206,209-212$ A representative DDS based on hollow gold nanoshells, reported by Chen and coll., ${ }^{202}$ is shown in Fig. 10. The core of the silica nanorattle was coated with a gold nanoshell, and then 35 functionalized with transferrin (Tf) and PEG. Silica nanorattles endowed the integrity with unique hollow structure and movable cores. On the other hand, the presence of gold nanoshells also endowed the hybrid nanostructure with tuneable optical property and facile access to surface functionalization. Based on these 40 advantages from different components, this DDS effectively combined the active and passive targeting, remote-controlled 
photothermal therapy, together with chemotherapeutic agents to completely kill tumour cells with NIR light irradiation.

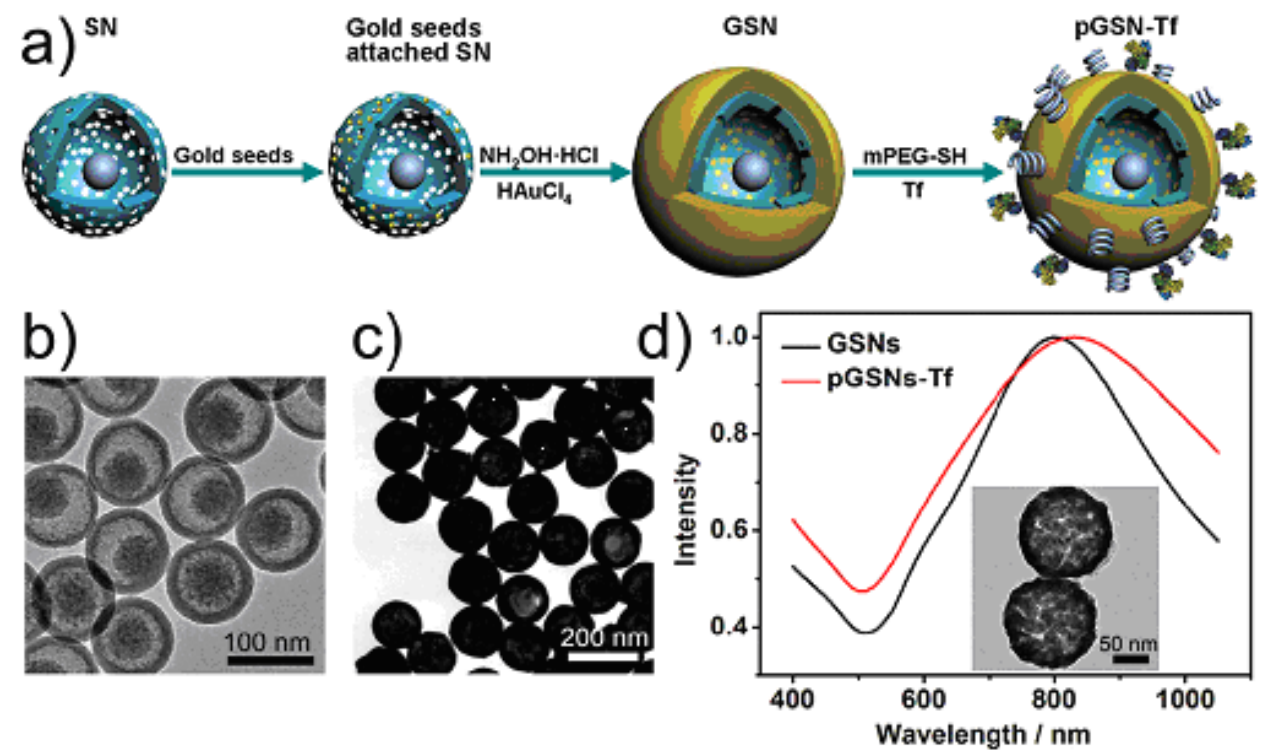

Fig. 10 a) Schematic diagram of gold-coated silica nanorattles (GSNs) synthesis and bio-conjugation; b) TEM image of silica nanorattles (SNs); c) TEM 5 image of GSNs and d) extinction spectra of the GSNs after PEGylation (pGSNs) and marking with targeting ligands of transferrin (pGSNs-Tf), the inset is the TEM image of pGSNs-Tf. Adapted with permission from Ref. 202. Copyright 2012 WILEY-VCH Verlag GmbH \& Co. KGaA, Weinheim..

Recently, Duan and coll. ${ }^{213}, 214$ reported a new strategy to fabricate hollow nanostructures assembled from amphiphilic gold 10 nanocrystals with mixed polymer brush coatings. Specifically, gold nanocrystals, nanospheres or nanorods, were immobilized with two types of chemically distinct polymer grafts, hydrophilic PEG and hydrophobic poly(methyl methacrylate) $)^{214}$ or polylactic $\operatorname{acid}^{215}$ poly(2-nitrobenzyl acrylate) ${ }^{216}$. The amphiphilicity-driven 15 self-assembly of block copolymers occurred in aqueous medium, leading to the formation of vesicular nanostructures. At the same time, the strong inter-particle plasmonic coupling leads to collective properties different from those in discrete units. Upon exposure to NIR light $\left(785 \mathrm{~nm}, 2 \mathrm{~W} / \mathrm{cm}^{2}\right)$, light-induced 20 deconstruction of the vesicles was confirmed by UV/vis spectroscopy and TEM, suggesting a great promise in NIRtriggered drug release. ${ }^{215}$

\section{Mechanisms of the triggered release via remote ${ }_{25}$ activation}

Generally, the application of remote triggers, e.g. AMF, NIR and ultrasound, is accompanied with local heating, which might also lead to various effects on the chemical/physical response, such as cleavage of thermo-labile covalent bonds, thermo30 induced phase change, thermo-induced structural deformation disintegration of nanostructures, etc. In this section, we detail item by item the underlying mechanisms involved during those remotely triggered release behaviours, even though for some specific nanostructures, there might be more than one mechanism 35 involved. For example, the triggered release upon fusion of PCMs might be attributed to both thermo-induced phase change and increased diffusion coefficient at higher temperature. Nevertheless, in each catalogue, we discuss the main mechanism, or at least the one taking the dominant role during the process of
40 remotely triggered release.

\section{1. Enhanced diffusion or permeation}

As detailed in Section 2, thermal and/or mechanical effects are involved during the application of remote triggers. Generally, 45 an increase in local temperature could contribute to an enhanced diffusion of the chemotherapeutic molecules and also permeability of the nanostructures by: i) increasing the kinetic energy and diffusivity of chemotherapeutic molecules, ii) facilitating the entering of chemotherapeutic molecules within the 50 disease microenvironments, and iii) enhancing blood circulation in the treated disease areas (in vivo practice). ${ }^{128}$ This enhanced diffusion or permeation effect ultimately leads to an improved therapeutic efficiency. The local heating under remote triggers can be tuned through experimental parameters, such as not only ${ }_{55}$ the application time, but also the wavelength and power of the NIR laser, frequency and magnetic field amplitude of AMF, and frequency, intensity or focus of the ultrasound setup. Therefore, the enhanced permeation or diffusion of chemotherapeutic reagents could also be manipulated via such kind of external 60 controls. For all those hybrid nanostructures targeted for remotely triggered release, the DDS is always accompanied with avoidable local heating during the remote stimuli application, which can definitely contribute to enhanced permeation or diffusion of the payloads. Here, in this section, we mainly focus on those DDS, 65 presenting only enhanced permeation or diffusion under remote triggers, without the synergetic contribution from other mechanisms, which are referred in the following sections.

For hybrid liposomes-based nanovehicles, when non-thermosensitive lipids were used, enhanced diffusion or permeation 70 represents the dominant mechanism behind the remotely stimulitriggered drug release. For example, in the work of Chu and 


\section{Cite this: DOI: 10.1039/c0xx00000x}

\section{www.rsc.org/xxxxxx}

ARTICLE TYPE

coll.,217 the liposomes@ $\mathrm{SiO}_{2} @ \mathrm{Au}$ nanovehicles were fabricated to deliver DOX. It was demonstrated that NIR laser could be used to excite the gold nanoshell and activate the drug release, thanks to the local heating. Other hybrid liposomes based on non5 thermo-sensitive lipids were also reported, such as the reports by Jin and coll. ${ }^{218}$, and Stroeve and coll., ${ }^{217}$ to cite only a few. Similar nanostructures, built with stimuli-responsive inorganic
NPs and non-thermo-sensitive organic components, have also been reported to remotely trigger the release based on such 10 mechanism, such as mesoporous silica embedded with gold nanorods, ${ }^{165}$ polymer micelles encapsulated with UCNPs, ${ }^{116}$ PVOH-based nano-capsules embedded with magnetic nanoparticles, ${ }^{219}$ etc. (a)
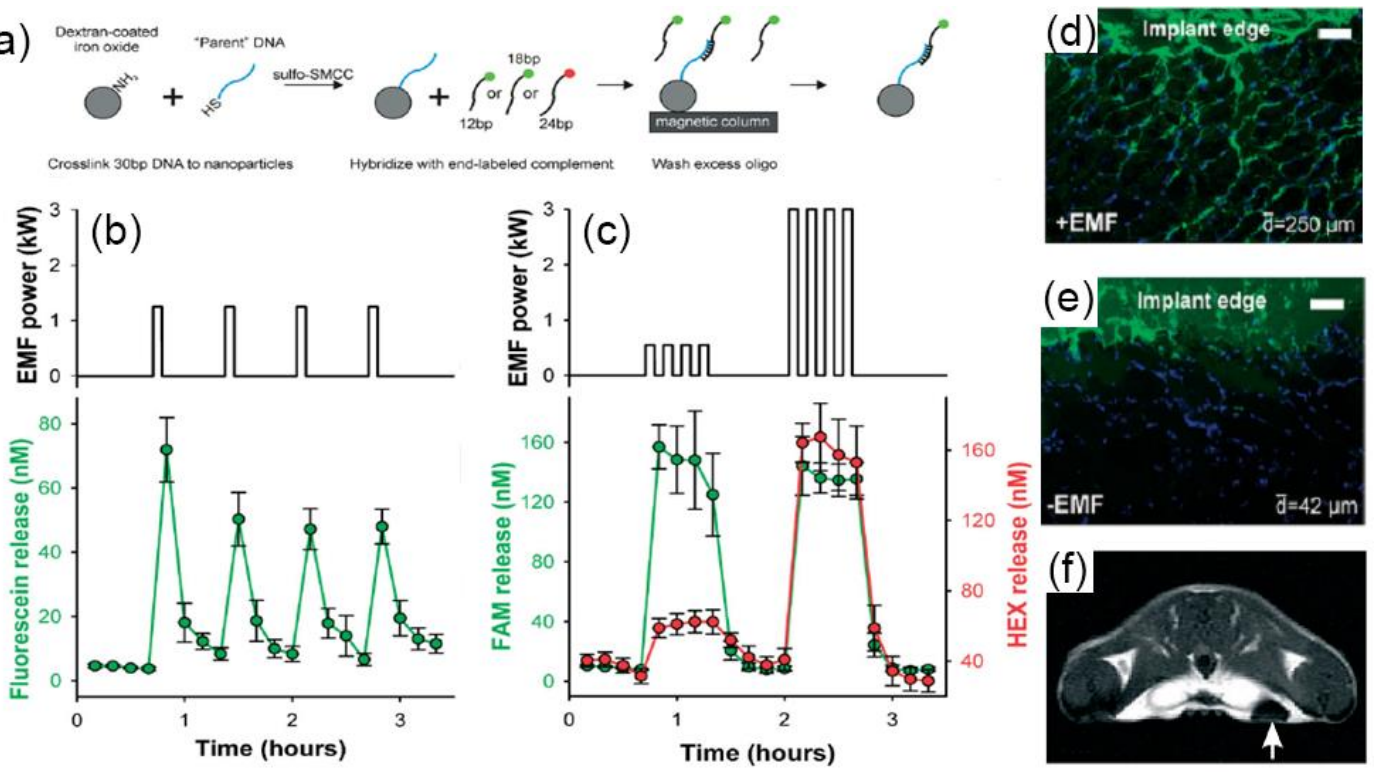

Fig. 11 Electromagnetic field (EMF) triggered release of guest-tethered nanovehicles in pulsatile and multistage profiles. (a) Nanovehicles were assembled by first covalently linking a 30 bp "parent" strand to a dextran-coated magnetic nanoparticle, and then allowing a fluorescent complement (of 12,18 , or $24 \mathrm{bp}$ ) to hybridize; (b) repeated EMF pulses of 5 minutes resulted in corresponding release of fluorescein guest molecules; (c) alteration of oligonucleotide duplex length shifted response of heat-labile tether enabling complex, tuneable release profiles. Low power EMF exposure resulted in 20 release of FAM-conjugated $12 \mathrm{bp}$, whereas higher power results in simultaneous melting of both 12 bp and 24 bp. Application of EMF to implants containing $18 \mathrm{bp}$ tethers resulted in release of model drugs and penetration far into surrounding tissue (D) when compared to unexposed controls (E) (scale bar: $100 \mu \mathrm{m}$ ). These mice were imaged with a 7T MRI scanner, and transverse section (F) depicts image contrast due to presence of nanoparticles (arrow). Adapted with permission from Ref. 66. Copyright 2007 WILEY-VCH Verlag GmbH \& Co. KGaA, Weinheim.

\section{4. 2. Thermo- or photo-labile bond cleavage}

One of the most facile strategies, involving the triggered release upon remote triggering, is to fabricate host/guest conjugates, in which the guest molecules are immobilized on the inorganic matrix via thermo-labile bonding. Different kinds of 30 nanovehicles based on the host/guest conjugate nanostructures have been presented in Section 3.1, and most of them are targeted for gene delivery. To some extent, the Watson-Crick base-pairing is not an exactly covalent bonding, however, still different from those conventional hydrogen bonds, since it highly depends on 35 the base-pair number, energy needed to break such kind of interaction might be higher than that of conventional hydrogen bonding. ${ }^{52}$ On account of the significance of Watson-Crick basepairing in the gene delivery system, we discuss it together with the conventional thermo-labile covalent bonds.
40 This concept is illustrated by $\mathrm{Fe}_{3} \mathrm{O}_{4} @$ DNA nano-conjugates (Fig. 11a) reported by Bhatia and coll. ${ }^{66}$ Single strand DNA (ssDNA) moieties (labelled with fluorescence group) were anchored on the dextran-coated magnetic nanoparticles to form a hybridized bond, and then these nano-sized vehicles were used to 45 deliver 12,18 or 24 basepair (bp) complementary ssDNA. Since the hybridized dsDNA is thermo-sensitive, it can be dehybridized into ssDNA above a specific temperature, depending on the length and guanine/cytosine $(\mathrm{G} / \mathrm{C})$ pair content in the ssDNA chain. Thanks to the local heating generated by the magnetic 50 nanoparticles under external AMF, the remotely AMF-triggered release of DNA can be achieved, depending on the magnetic field strength, namely the local temperature generated. Pulsatile release of fluorescence-labelled ssDNA was also evidenced under EMF $(400 \mathrm{kHz}, 0.55 \mathrm{~kW})$ on/off cycles in Fig. 11b. Moreover, by 55 immobilizing two different DNA moieties, 12 and 24 pb ssDNA, 
a selective triggered release was obtained under lower field strength $(400 \mathrm{kHz}, 0.55 \mathrm{~kW})$, while non-selective triggered release under high field strength $(400 \mathrm{kHz}, 3 \mathrm{~kW})$, as shown in Fig. 11c. In vivo test of the EMF-triggered release of model drugs, 5 as well as the penetration far into surrounding tissue, was confirmed via confocal microscope observation (Fig. 11d and 11e). Moreover, the iron oxide NPs could also act as contrast agent for biomedical imaging and/or in vivo delivery tracking (Fig. 11f). A similar remote release of DNA through 10 dehybridization via AMF application was also reported by Baglioni and coll. ${ }^{67}$ based on the GNPs@ $\mathrm{CoFe}_{2} \mathrm{O}_{4} \mathrm{NP}$ and immobilized oligonucleotides. There have been also some successful attempts dealing with the thermo-labile hybridization of double-stranded oligonucleotides, aiming for the remotely 15 NIR-triggered release of single-stranded oligonucleotides immobilized on GNRs, ${ }^{217}$ gold nanoshells ${ }^{55,57,59,60,65,169}$ and gold nanospheres, ${ }^{56,61}$ etc.

Another thermo-labile covalent bond aimed for remotely triggered gene release is the $\mathrm{Au}-\mathrm{S}$ conjugation. Based on this 20 strategy, sulfur-bearing guest molecules to be delivered are, in most cases, directly immobilized onto gold matrix via $\mathrm{Au}-\mathrm{S}$ conjugation. In the report of Chen and coll., ${ }^{220}$ GNRs@DNA conjugates (gold nanorods and gene of enhanced green fluorescence protein) were exposed to femtosecond laser pulses. ${ }_{25}$ Cleavage of the Au-S bonding resulted in the release of preloaded DNA molecules. On the other hand, this treatment was also accompanied with the shape transforming of gold nanorods, due to the higher temperature generated during NIR laser irradiation. This kind of release mechanism was also applied in 30 other conjugates consisting of gold nanoparticles and DNA molecules. ${ }^{49,}{ }^{51-54}$ It is deserved to note that, in the report of Hamad-Schifferli and coll., ${ }^{52}$ they immobilized two different DNA molecules onto two kinds of GNRs with different aspect ratios (corresponding to different SPR bands), respectively. ${ }_{35}$ Similarly to the selective release reported by Bhatia and coll., ${ }^{66}$ the selectively triggered release of the DNA molecules was also achieved by controlling the NIR irradiation wavelength, according to the two different SPR bands.

A similar thermo-labile bonding was also reported by 40 Niidome and coll. ${ }^{69}$ on the immobilization of guest molecules onto the surface of magnetic nanoparticles via the Diels-Alder reaction. The Diels-Alder reaction is known as a reversible cycloaddition reaction between diene and alkene groups to form a cycloadduct. In particular, the reaction between furan and 45 maleimide proceeds at $60{ }^{\circ} \mathrm{C}$, and its reverse reaction, the socalled retro-Diels-Alder reaction, occurs at $90{ }^{\circ} \mathrm{C}$. In that work, a building block of Diels-Alder cycloadduct with PEG chain and a thiol end-group (mPEG20,000-DA-SH) was synthesized from the Diels-Alder reaction between $\mathrm{mPEG}_{20,000}$-maleimide and furfuryl ${ }_{50}$ disulfide, and the subsequent reduction from disulfide bonds to thiol groups with tris(2-carboxyethyl)phosphine hydrochloride. The cycloadduct of $\mathrm{mPEG}_{20000}-\mathrm{DA}-\mathrm{SH}$ was then immobilized onto the GNRs, and remotely NIR-triggered release of PEG was detected, when the temperature of the system reached $80{ }^{\circ} \mathrm{C}$ 55 (local temperature was supposed to reach above $90{ }^{\circ} \mathrm{C}$ ). Another thermo-labile azo-groups, reported by Pellegrino and coll., ${ }^{68}$ was used to immobilize guest molecules onto the surface of iron oxide nanoparticles. Upon remote AMF treatment, the azo-groups were cleaved. Based on this strategy, the mass ratio between the ${ }_{60}$ delivery vehicle and the drug was significantly reduced, and both hydrophobic and hydrophilic drugs were released. Moreover, combination of different drugs bound on the same nanoparticle at different distances was also confirmed to realize the independent and selective release of each chemotherapeutic payload.

65 UNCPs are capable of converting continuous NIR light into a range of different wavelengths throughout UV, visible and NIR regions. This attractive capability makes it possible to cleave UVlabile chemical bonding, such as benzoin and $o$-nitrobenzyl derivatives and coumarin, under NIR irradiation. ${ }^{221}$ Xing and 70 coll. $^{222}$ firstly utilized this strategy to trigger the release of siRNA from the silica-coated UNCPs, through NIR (980 nm)-induced cleavage of $o$-nitrobenzyl ester bonds. Similar strategy was also extended in the work of Zhang and coll. ${ }^{223}$ for NIR-triggered release NO, Branda and coll. ${ }^{224}$ for NIR-triggered release of 75 carboxylic acid, Zhang and coll. ${ }^{169}$ for triggered release of DNA. Yeh and coll. ${ }^{225}$ for NIR-triggered release of 2nitrobenzaldehyde, Xing and coll. ${ }^{226}$ for NIR-triggered release of $D$-luciferin, Krull and coll. ${ }^{227}$ for NIR-triggered release of 5fluorouracil, $\mathrm{Li}$ and coll. ${ }^{221}$ for NIR-triggered release of 80 chlorambucil, based on the combination of UNCPs-containing hybrid nanostructures, photo-labile covalent bonding and remote NIR irradiation. Therefore, in light of the toxicity essence and limited penetration depth of UV irradiation, a rational combination of UNCPs and NIR irradiation could effectively 85 solve these puzzles, and open up a favourable situation for more conventional UV-sensitive DDS.

The major advantage for thermo- or photo-labile bond cleavage mechanism might underlie on the simple and facile fabrication routes needed to fabricate the host/guest conjugates, 90 compared with other complex nanostructures. Moreover, it is easier to obtain a higher and controllable loading capacity. However, there might be still some concerns over the further application of the host/guest conjugates based on the mechanism of covalent bond cleavage. First of all, the toxicity or activity of 95 the chemotherapeutic agents immobilized on the surface could not be totally quenched during the in vivo delivery. Second, for most of the systems developed, high local temperature was needed to cleave the thermo-labile covalent bonding. The more stable the covalent bonding, the less the premature release during 100 the delivery routine. However, higher power and/or period of treatment for remote stimuli application are needed to cleave these thermo- or photo-labile bonds. Third, this triggered release mechanism is also challenged by some other drawbacks, such as the local overheating, thermo- or photo-induced degradation of 105 the chemotherapeutic agents, limited therapeutic efficiency, shape transformation or dissociation of the nanostructures, etc. In order to find clinical use of these host/guest conjugates, further optimization/consideration of their structures and also elaborated selection of thermo- or photo-labile bonds, as well as the remote 110 energy matching the release, are needed.

\section{3. Fusion of phase-changed materials}

As discussed in Section 3. 6 on the gating systems based on porous nanostructures, different strategies are attempted to 115 engineer them with gate-keeping components, in order to achieve a "zero premature release" and also triggered release upon 


\section{Cite this: DOI: $10.1039 / \mathrm{c0xx00000x}$}

\section{www.rsc.org/xxxxxx}

ARTICLE TYPE

different stimuli. These gating systems have already been particularly summarized in the recent review of Zink and coll., ${ }^{159}$, ${ }^{160}$ Lin and coll., ${ }^{154,158}$ and Martinez-Manez and coll. ${ }^{2}$ Among the gate-keeping components, thermal-induced PCMs, such as fatty 5 acid or fatty alcohol, were first reported by Xia and coll. ${ }^{198}$ In this work, 1-tetradecanol $\left(T_{\mathrm{m}}\right.$ of $\left.38-39{ }^{\circ} \mathrm{C}\right)$ was used as gatekeeper for these preloaded guest molecules within gold nanocages, taking advantage of its immiscibility with water as well as good biocompatibility (Fig. 12a). Since a PCM can reversibly change 10 its physical state between solid and fluid within a narrow temperature range, thus it could confine the preloaded guest molecules within the gold nanocages until the local temperature exceeded the $T_{\mathrm{m}}$ (Fig. 12b-d). As shown in Fig. 12e, mixture of Rhodamine 6G (R6G) and 1-tetradecanol was loaded together 15 within the gold nanocages with minimal release behaviour.
However, sustainable release was detected, once the temperature was increased above $T_{\mathrm{m}}$ of 1-tetradecanol. Thanks to the reversible phase transition of the PCMs, an on/off control over the release could be easily achieved via the heating on/off 20 treatment (Fig. 12f), in a pulsatile manner. Moreover, as a remote and deeply penetrating energy source, high-intensity focused ultrasound (HIFU) can be used to trigger the release (Fig. 12g). More recently, Singamaneni and coll. ${ }^{199}$ extended this research, but used NIR in this work to trigger the release of Nile red or ${ }_{25}$ DOX from the same system. Additionally, they introduced surface enhanced Raman spectroscopy (SERS) to monitor the release. Considering the absence of strong absorption in the NIR range for chemotherapies, e.g. DOX, the SERS technique could be effectively used for in vitro/vivo tracing the release.

(a)
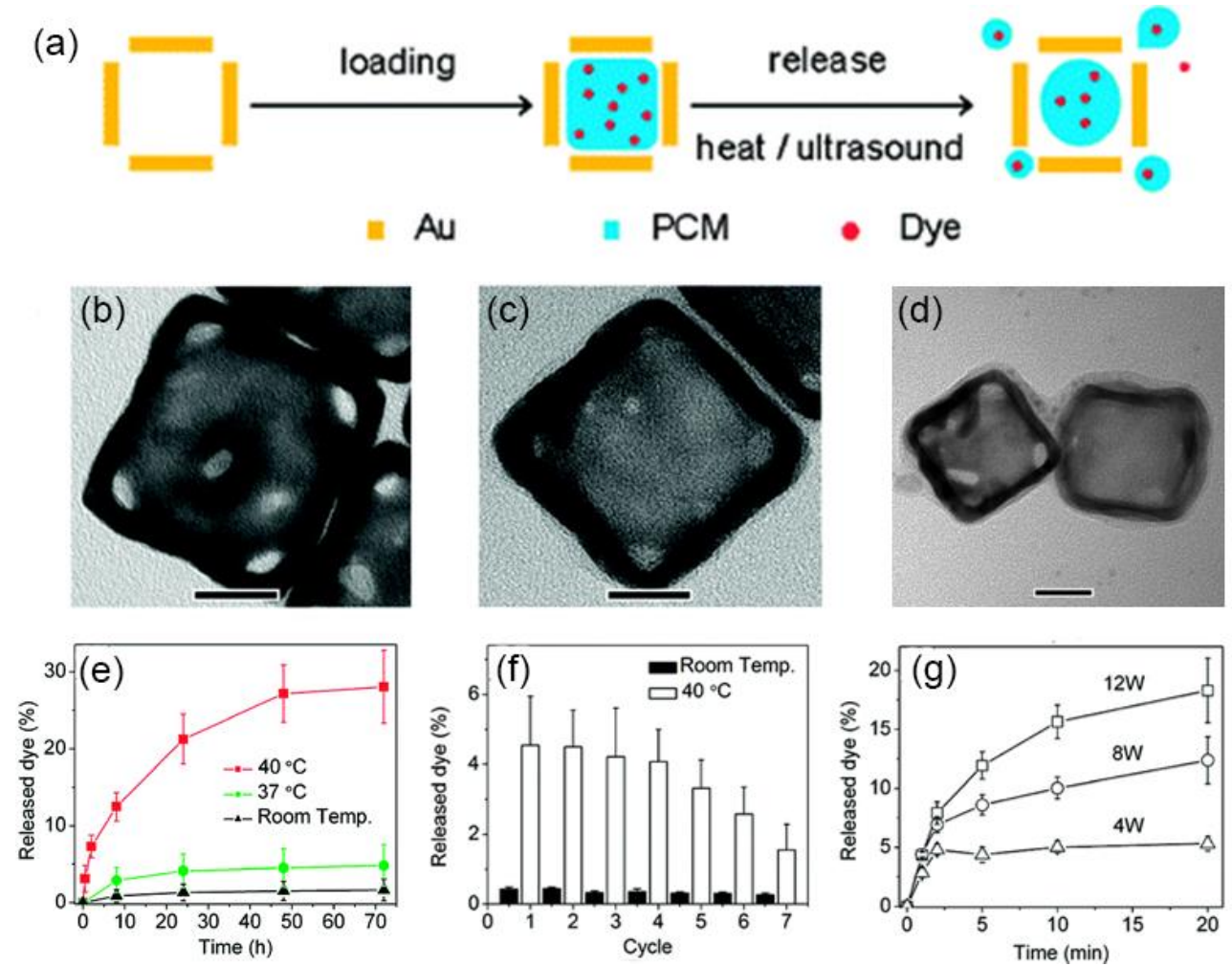

Fig. 12 Schematic illustration on how to load the hollow interior of a gold nanocages with a dye-doped PCM and then have it released from the gold nanocages by direct or ultrasonic heating (a); TEM image of pristine gold nanocages (b), drug loaded nanogels before (c) and after (d) HIFU application. Release profiles of R6G under direct heating to various temperatures for different periods (e); pulsatile release under alternative heating/cooling cycles (f), and release profiles of R6G by HIFU at different applied powers (b); and (c). Adapted with permission from Ref. 198. Copyright 2011 American Chemical 35 Society.

Inspired by the great potential role of PCMs in gating system, Duguet and coll., recently reported the combination of PCMs (1tetradecanol) with $\gamma-\mathrm{Fe}_{2} \mathrm{O}_{3} @ \mathrm{MSNs}^{182}$ or GNR@MSNs ${ }^{183}$, with responsiveness to AMF and NIR light, respectively. The presence 40 of PCMs was again confirmed to realize a "zero-premature release", while triggered release could be readily obtained upon the application of AMF and NIR light. Furthermore, a "pulsatile" release, with control over the release amount/period, can be facilely achieved by manually control over the on/off remote 45 stimuli, indicating the possibility of releasing the uploaded chemotherapeutics following the biological rhythm. 


\title{
Journal of
}

\section{Materials Chemistry B}

\author{
Cite this: DOI: $10.1039 / \mathrm{c0xx00000x}$
}

PAPER

(a)
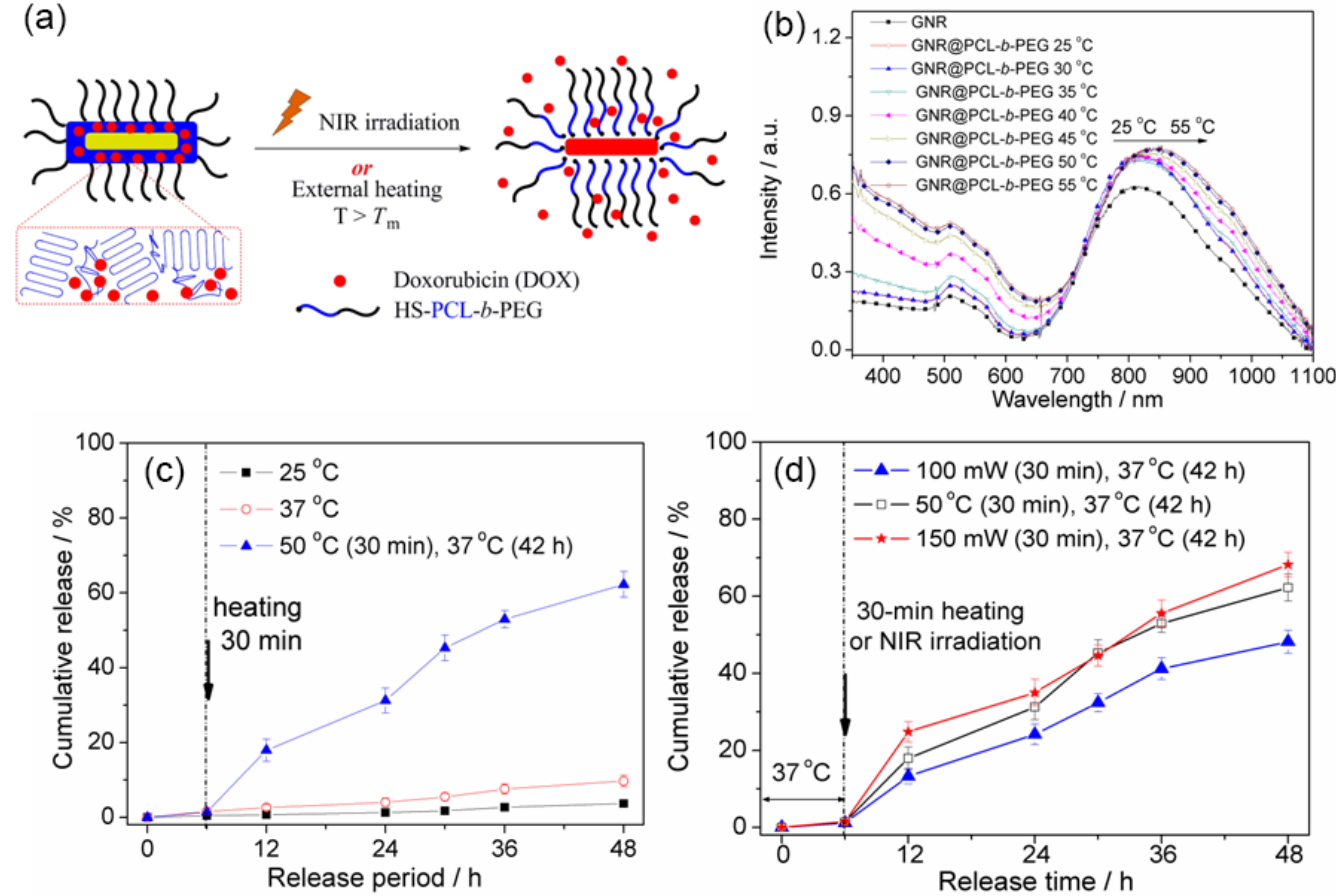

Fig. 13 Preparation of GNR@PCL- $b$-PEG, drug loading of DOX and triggered release of DOX by conventional external heating or internal heating induced by remote NIR irradiation (a); UV/Vis spectra of an aqueous suspension of GNR @ PCL- $b$-PEG (50 $\mu \mathrm{g} \mathrm{mL}^{-1}$ ) under different temperatures (b); release profiles of DOX from a DOX-loaded aqueous suspension of GNR@PCL-b-PEG $\left(150 \mu \mathrm{gL}^{-1}\right)$ at different temperatures (c); release profiles of 5 DOX over $42 \mathrm{~h}$ at $37{ }^{\circ} \mathrm{C}$ after NIR-laser irradiation for $30 \mathrm{~min}(802 \mathrm{~nm}, 100$ or $150 \mathrm{~mW})$ (d). Adapted with permission from Ref. 88. Copyright 2014 WILEY-VCH Verlag GmbH \& Co. KGaA, Weinheim.

Crystalline polymer with the character of thermo-induced phase change might also be used for the remotely triggered 10 release. Jérôme and coll. ${ }^{88}$ reported the use of crystalline PCLbased amphiphilic block copolymers to form a phase-changed polymer corona over gold nanorods (Fig. 13a), whose SPR band located in the NIR range and could also be affected by the temperature (Fig. 13b). Hydrophilic drug, e.g. DOX, was loaded 15 within the hydrophobic PCL domain and trapped among those PCL crystallites. As reported, a minimal premature release $(<5 \%$ entire payload) was detected within $48 \mathrm{~h}$ at $37{ }^{\circ} \mathrm{C}$ (Fig. 13c). However, upon the remote NIR application, released DOX was detected due to the fusion of PCL crystallites resulting from the ${ }_{20}$ NIR-induced local heating, and also heating up of nanovehicles might also contribute to a faster diffusion of cargo molecules (Fig. 13d). Similar work was also reported by Zhong and coll. ${ }^{86}$, ${ }^{87}$ As discussed in Section 3.5 on hybrid liposome nanostructures, thermo-sensitive liposomes (TSLs) could release the drug ${ }_{25}$ payloads above Tm of the lipid bilayers. Phase change of the lipid membrane occurs above $T_{\mathrm{m}}$, from a well-ordered gel phase to the less ordered liquid crystalline phase. Liposomal membranes in the gel (i.e. solid-like) phase are less permeable to water and cargo molecules, compared to the liquid-crystalline phase. ${ }^{128,} 129$ 30 As reported by Drummond and coll., ${ }^{125}$ the membrane permeability above $T_{\mathrm{m}}$ increased by several orders of magnitude, thereby facilitating the cargo release. Rational combination of inorganic heating generators with phospholipid molecules, which possess $T_{\mathrm{m}}$ just above the body temperature, is also receiving 35 increasing attention in clinical practices, on account of the facile access to local hyperthermia and to substantial improvements over the controlled release behaviours.

A representative DDS based on TSLs and gold nanoparticles for remotely triggered release is reported by Romanowski and ${ }_{40}$ coll. ${ }^{145}$ As shown in Fig. 14a, a gold nanoshell was formed with gold nanoparticles over the TSLs made of dipalmitoyl phosphatidylcholine (DPPC), and a remotely triggered release was achieved with the NIR-induced phase change of the TSLs. Thermo-induced release evidenced ca. $24 \%$ release at $31{ }^{\circ} \mathrm{C}$ but ${ }_{45} 100 \%$ near $47{ }^{\circ} \mathrm{C}$ under gradual heating (Fig. 14b), in agreement with the pre-transition temperature at $35.3{ }^{\circ} \mathrm{C}$ and a main phase transition at $41.4{ }^{\circ} \mathrm{C}$ of DPPC. While for the light-triggered release, the responsiveness of liposome to different-wavelength light was modelled with a four-parameter logistic function, with 


\section{Cite this: DOI: 10.1039/c0xx00000x}

\section{www.rsc.org/xxxxxx}

ARTICLE TYPE

$50 \%$ release observed at 12 , and $82 \mathrm{~J}$ of cumulative energy for on-resonant (971 nm, Fig. 14c) and off-resonant (655 nm, Fig. 14d), respectively. For the sake of comparison, the release increased slowly over a broad range of energy for bare liposomes 5 without gold nanoshells (Fig. 14e). Moreover, temperature of gold-coated liposomes (Fig. 14f and $\mathbf{1 4 g}$ ) rose more steeply and reached a higher temperature than bare liposomes (Fig. 14h).

Based on the same principle of rationally combining inorganic heating generators and phospholipid molecules, similar 10 works on TSLs@gold nanovehicles ${ }^{143}, 144,148,149,153,207$ for remotely NIR-triggered release and TSLs@iron oxide nanovehicles $44,129,135,137-139,141$ for remotely AMF-triggered release were also reported. Moreover, there are also some reports on nanovehicles based on the combination of TSLs and gold or 15 magnetic nanoparticles, for remotely ultrasound-triggered release. In this case, the light- (gold nanoparticles) or magnetismresponsive (magnetic nanoparticles) components will not serve as heating generators anymore, but act as the heat conductors or transistor of ultrasonic energy. For example, DDS consisted of 20 TSLs, iron oxide and chemotherapeutic reagents were reported also for remotely ultrasound-triggered release.228, 229 More examples could be referred to the reviews on magnetic liposomes for MRI diagnosis ${ }^{27}$ or magnetic field-guided delivery. ${ }^{129}$
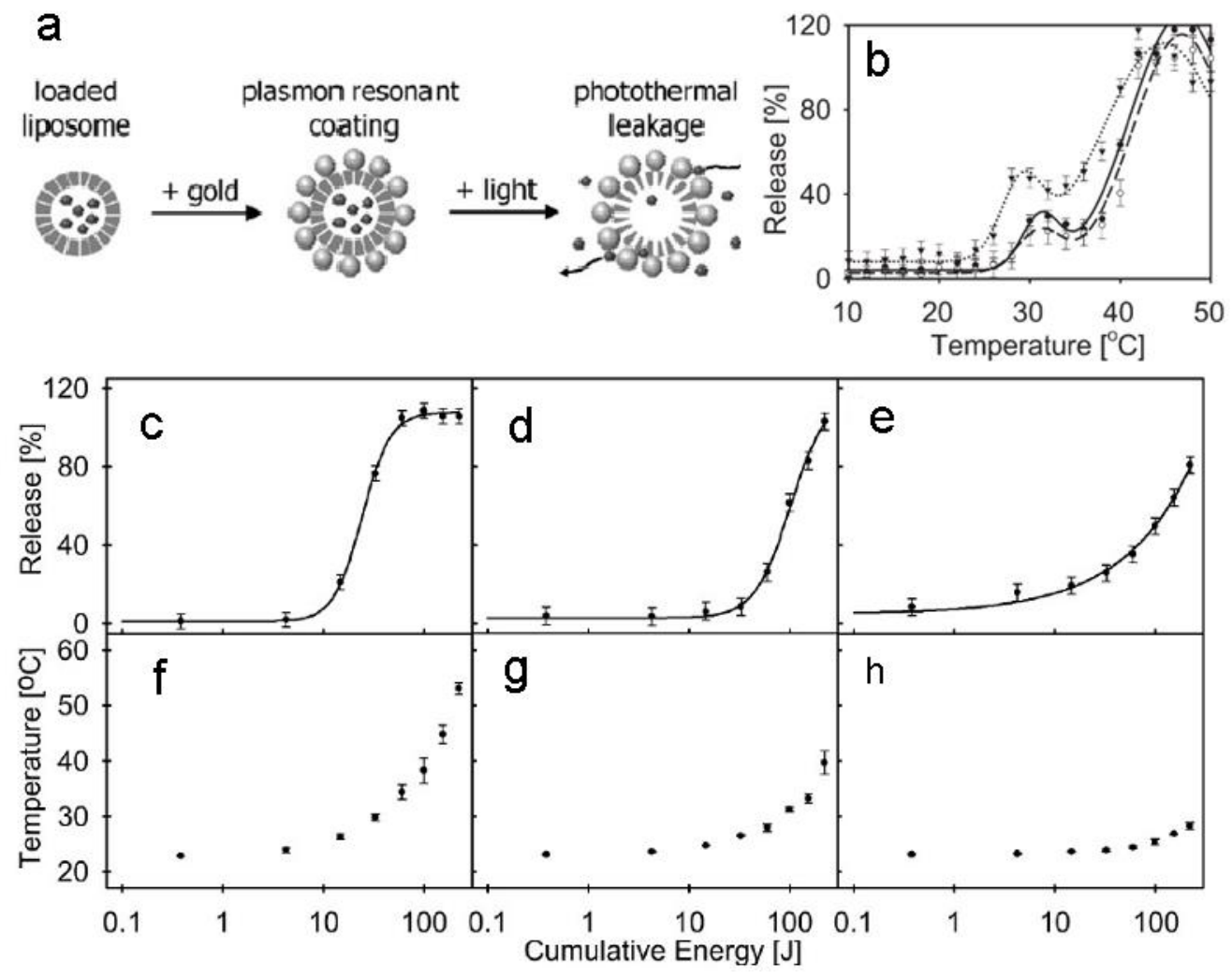

Fig. 14 Schematic representation of the formation of plasmon-resonant shells and subsequent content release: hydrophilic cargoes were encapsulated in thermo-sensitive liposomes, and gold coating was introduced by reduction of ionic gold in the presence of liposomes. When illuminated at the wavelength matching the plasmon resonance, the energy of absorbed light was converted into heat, so the thermo-sensitive liposomes release their content. (a) Thermo-induced release behaviours of bare liposomes (inverted triangle, dotted), gold-coated liposomes resonant at 655 nm (open circle, dashed), and 30 gold-coated liposomes resonant at $971 \mathrm{~nm}$ (closed circle, solid line). (b) Light-induced release behaviours for gold-coated liposomes resonant at $971 \mathrm{~nm}$ (c), $655 \mathrm{~nm}(\mathrm{~d})$, and bare liposomes (e); and peak temperatures recorded during light-induced release, for gold-coated liposomes resonant at $971 \mathrm{~nm}$ (f), $655 \mathrm{~nm}$ (g), and bare liposomes (h). Adapted with permission from Ref. 145. Copyright 2009 WILEY-VCH Verlag GmbH \& Co. KGaA, Weinheim.

\section{4. Photo-induced isomerisation}

35 Light-responsive macromolecular colloids based on the photo-isomerizable moieties, such as azobenzene groups, have been widely studied in controlled drug release. ${ }^{230}$ In most of the studies, photochromic groups within these amphiphilic block copolymers experience a light-induced trans-cis transition. Upon 40 irradiation with low wavelength, e.g. $360 \mathrm{~nm}$ for azobenzene group, they are converted to an isomeric form, leading to the increased polarity and hydrophilic essence of the copolymer, 
followed with the destabilization of the micellar structures. However, reverse isomerization could be activated under irradiation with higher wavelengths, e.g. $440 \mathrm{~nm}$, resulting in the recovery of the former isomeric and hydrophobic essence, and the 5 re-formation of the micelle structures. ${ }^{230}$ This specific photoinduced trans-cis transition has been used to remotely triggered release from micelles, ${ }^{230}$ and mesoporous silica. ${ }^{231}$ In addition to these azobenzene moieties, similar photo-isomerizable moieties, such as cinnamic acid groups, and other photo-cleavable 10 moieties, e.g. benzoin derivatives ${ }^{224}$ and $o$-nitrobenzyl groups, ${ }^{34}$ have also found application in light-triggered release. The major advantage of DDS based on the photo-isomerizable moieties is the reversible nature, allowing turning the systems on and off, and releasing the cargo molecules on demand. ${ }^{231}$ Even though 15 these light-responsive components exhibit great promise in DDS and there have been a large variety of DDS based on these structures reported, they still suffer a lot from the lack of in vivo translation; essentially because of the damages to healthy cells from UV irradiation can not be avoided. Moreover, the UV light 20 can not penetrate into the tissue. As declared in Section 1, the irradiation with UV light is not in the scope of this review, thus those triggered release based on UV-triggered trans-cis mechanism is not highlighted here, but more information can be referred to the tutorial review by Zhao and coll. ${ }^{230}$ (a)
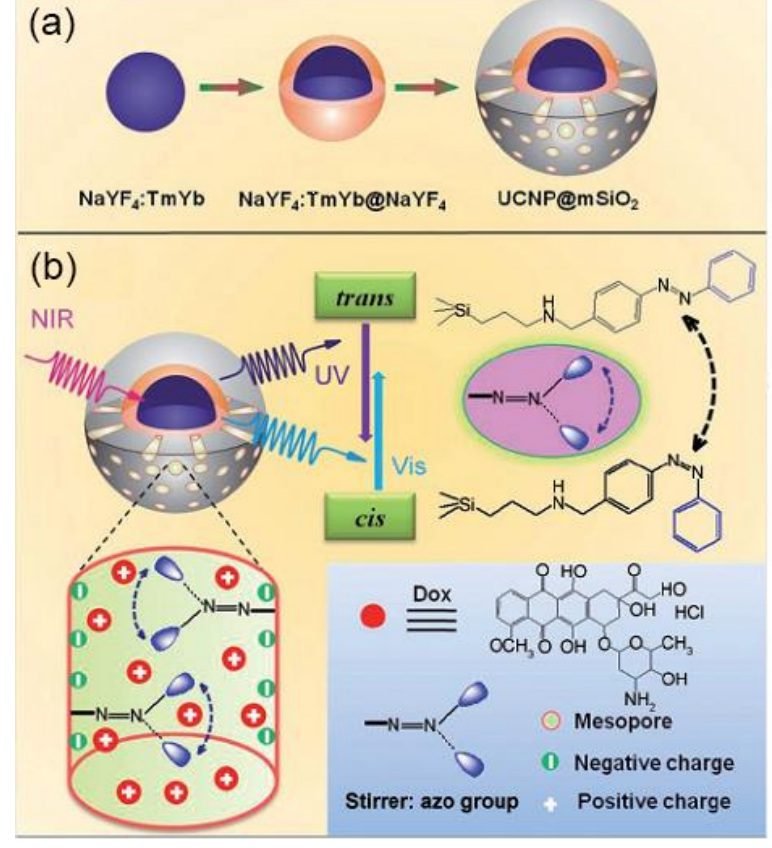
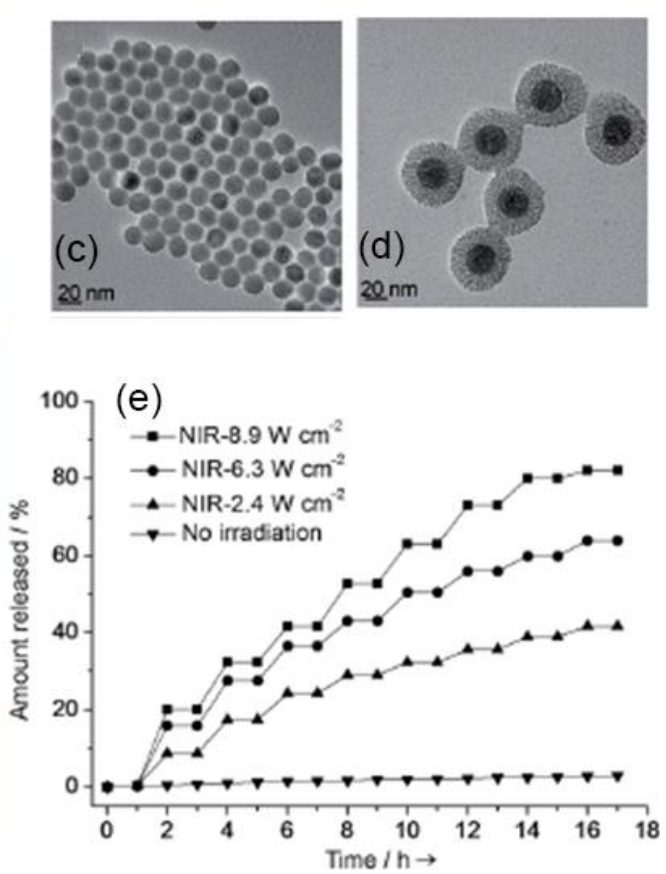

Fig. 15 a) Synthetic procedure for upconverting nanoparticles coated with a mesoporous silica outer layer (a), NIR light-triggered DOX release by making use of the upconversion property of UCNPs and trans-cis photoisomerization of azobenzene moieties introduced in the mesopore network of a mesoporous silica layer (b). TEM images of $\mathrm{NaYF}_{4}: \mathrm{Tm}, \mathrm{Yb}$ (c) and $\mathrm{NaYF}_{4}: \mathrm{Tm}, \mathrm{Yb} @ \mathrm{NaYF}_{4} @ \mathrm{mSiO}_{2}$ (d); drug release in PBS under NIR light irradiation $30\left(980 \mathrm{~nm}, 8.9 \mathrm{~W} \mathrm{~cm}^{-2}, 1 \mathrm{~s}\right)$ and dark conditions, alternatively (e). Adapted with permission from Ref. 232. Copyright 2013 WILEY-VCH Verlag GmbH \& Co. KGaA, Weinheim.

In spite of the critical limitation for those UV-sensitive DDS in potential clinical practices, a new revolution, fortunately, is 35 brought up after the report of upconverting nanoparticles (UCNPs), which are consisting of $\mathrm{NaYF}_{4}$ nanocrystals doped with $\mathrm{Tm}^{3+}$ and $\mathrm{Yb}^{3+} .233,234$ This kind of nanoparticles manifests themselves with the prominent performance as light-harvesting antennae, and sequentially absorbing multiple photons of NIR

40 light and converting them into higher energy (UV light). ${ }^{233}, 234$ This new strategy could be an effective alternative to resolve the as-referred drawbacks, and offers a totally new platform, once some UCNPs are incorporated. The first example of using UCNPs for NIR-triggered release was demonstrated by Branda 45 and coll. ${ }^{224}$ for the cleavage of photo-labile 3',5'-dialkoxybenzoin derivatives and release of acetic acid-group. After this report, the same group extended this strategy on NIR-triggered cargo release from micelle nanostructures, which bear photo-labile moieties of $o$-nitrobenzyl groups. ${ }^{115,116}$ The underlying mechanism is based 50 on the photo-induced cleavage of photo-labile bonds (Section 4.2).

More recently, the light-induced trans-cis mechanism, with the help of UCNPs, has just been further adapted to induce the NIR-triggered release from mesoporous silica by Shi and coll. 55 (Fig. 15). ${ }^{232}$ Core/shell mesoporous silica-coated UCNPs were first synthesized (Fig. 15a, c and d), and then the inner mesopores were modified with $N$-(3-triethoxysilyl)-propyl-4phenyl-azobenzamide to introduce azobenzene moieties. Thus, it is envisioned that the reversible photo-isomerization by ${ }_{60}$ simultaneous UV/vis light emitted by the UCNPs could create a continuous rotation-inversion movement. The back and forth wagging motion of the azobenzene moieties could act as a molecular impeller that propels the release of DOX (Fig. 15b). The proof-of-concept release showed that the DOX release 


\title{
Cite this: DOI: 10.1039/c0xx00000x
}

\author{
www.rsc.org/xxxxxx
}

ARTICLE TYPE

amount reached $40 w t . \%$ in $16 \mathrm{~h}$ under intermittent NIR exposures $\left(980 \mathrm{~nm}, 2.4 \mathrm{~W} / \mathrm{cm}^{2}\right)$, and reached a maximum of 80 $w t$. \% after 16-h laser irradiation $\left(8.9 \mathrm{~W} / \mathrm{cm}^{2}\right)$. However, without NIR irradiation, less than $5 w t$. \% of DOX was leached into the 5 aqueous medium in $17 \mathrm{~h}$ (Fig 15e), indicating that the increased release rate is attributed to the external NIR irradiation, and also depends on the irradiation powers. In another recent report, $\mathrm{Qu}$ and coll. ${ }^{235}$ intricately integrated the hollow UNPs and photosensitive merocyanine moieties to build a smart DDS for 10 remotely triggered release of protein. Due to the isomerization of merocyanine (positively charged) to spiropyran (neutral) under NIR irradiation, the pre-loaded proteins, trapped via electrostatic interaction between protein and MC moieties, can be easily released.

15

\section{5. Thermo-induced swelling/collapsing of thermo-} responsive polymer

\subsubsection{Sneezing out}

Thermo-responsive polymers, e.g. PNiPAAm-, PNVCL- or 20 PEO-PPO-PEO-based macromolecules, exhibit a LCST in aqueous medium. The macromolecular chains undergo a reversible coil/globule conformation transition around the LCST, during which the former hydrophilic and expanded conformation (below the LCST) evolves into hydrophobic and compact one 25 (above the LCST). ${ }^{2,}$ 6, 104, 236 As mentioned above, local heating could be generated upon exposure of specific inorganic components to remote stimuli. Thus, by engineering them with thermo-responsive polymers, it is expected that the application of remote stimuli could lead to the sharp shrinkage of the polymers 30 domains, followed with the sneezing out of guest molecules. Similar results have been reported when using PNiPAAm- ${ }^{79}, 93$ 101,237 or PEO-PPO-PEO-based ${ }^{76,122,238}$ hybrid nanostructures.
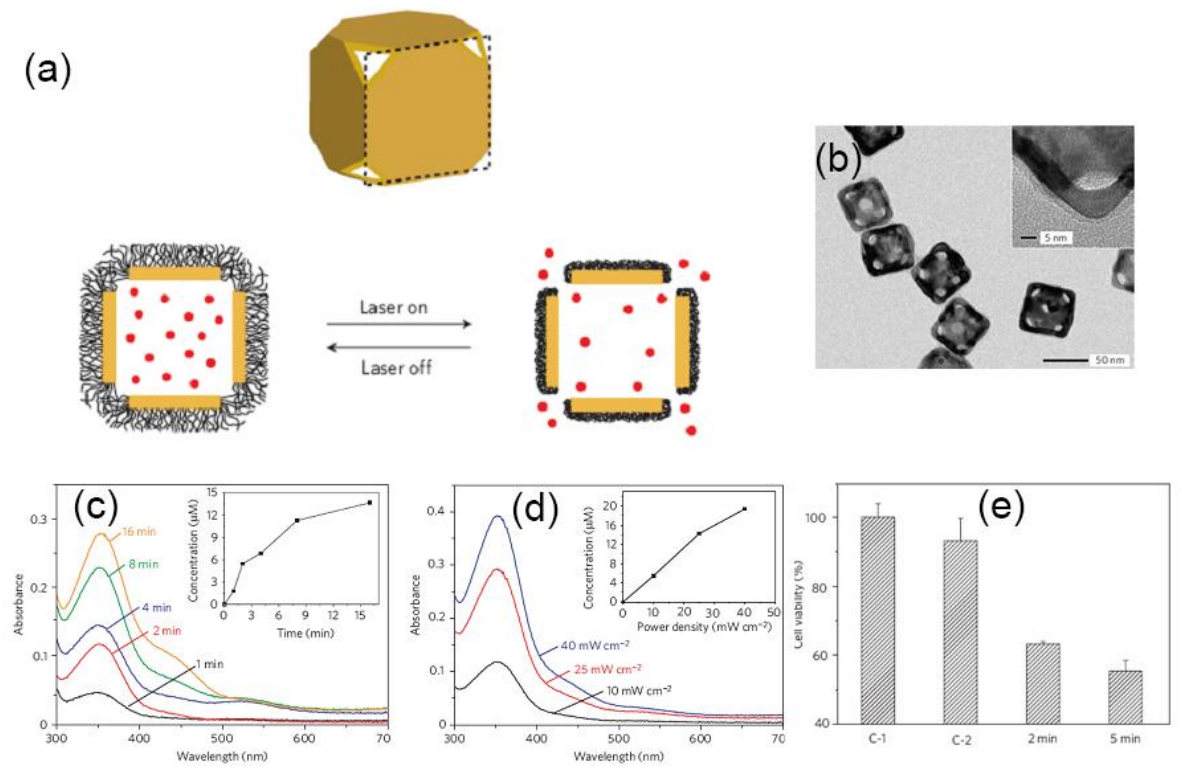

35 Fig. 16 (a) Gold nanocages-based DDS decorated by a thermoresponsive polymer: upon exposure to a NIR laser, energy absorbed by the nanocages was converted into heat, triggering the smart polymer to collapse and release the drug payload. When the NIR laser was turned off, the polymer chains reverted to the extended conformation and terminated release. (b) TEM images of gold nanocages with surface-coated by PNiPAAm-co-PAAm copolymer. (c) Absorption spectra of alizarin-PEG released from the gold nanocages at $42^{\circ} \mathrm{C}$; and by (d) exposure to 2 -min NIR irradiation at 10,25 and $40 \mathrm{~mW} \mathrm{~cm}^{-2}$. The insets show the concentrations of alizarin-PEG released from the nanocages under different conditions. (e) Cell viability after going through different 40 treatments: (C-1) 2-min NIR irradiation in the absence of gold nanocages; (C-2) 2-min NIR irradiation in the presence of Dox-free Au nanocages; and (2/5 $\mathrm{min}$ ) NIR irradiation for 2 and $5 \mathrm{~min}$ in the presence of Dox-loaded gold nanocages. A power density of $20 \mathrm{~mW} \mathrm{~cm}^{-2}$ was used for all of these studies. Adapted by permission from Ref. 195 from Macmillan Publishers Ltd: Nature Materials, copyright 2009.

\subsubsection{Opening the pores}

45 For hollow nanostructures, thermo-responsive corona on the external shell can act as a protective layer to prevent the premature release during the delivery. However, upon the application of remote stimuli, thermo-induced collapsing of the polymer corona might open the pores, resulting in the subsequent ${ }_{50}$ drug release. Xia and coll. reported the remotely triggered release with NIR irradiation ${ }^{195}$ or high-intensity focused ultrasound (HIFU) treatment ${ }^{194}$ from the gold nanocages. The gold nanocages were loaded with cargoes, whose external faces were 
coated with a thin layer of thermo-responsive $\operatorname{poly}(N$ isopropylacrylamide-co-acrylamide) [poly(NIPAm-co-AAm)] copolymer, with LCST tuneable between $32-50{ }^{\circ} \mathrm{C}$ depending on the chemical compositions (Fig. 16a-b). Since the 5 collapsing/swelling of the polymer is reversible, the fine-tuning of NIR power, HIFU intensity and treatment period enables to control the dosage release (Fig. 16c-d). Moreover, the triggered release has been demonstrated to be applicable with fluorescently labelled proteins, drugs, and enzymes, and in vitro NIR-triggered 10 release of DOX was also confirmed via the combination of the culture of breast cancer cells and NIR irradiation (Fig. 16e).

More recently, Lin and coll. ${ }^{170}$ also reported DDS based on hollow nanostructures with a thermo-responsive polymer coating. In their work, UCNPs nanoparticles were coated with 15 mesoporous silica shell. Then a thermo-responsive polymer corona was formed with poly[( $N$-isopropylacrylamide)-co(methacrylic acid)] [P(NiPAAm-co-MAA)] copolymers, as an active valve to moderate the diffusion of cargoes from the internal cavities. The pre-loaded DOX molecules can be safely 20 trapped with a low-level leakage at low temperature or/and high $\mathrm{pH}$ value, but significantly enhanced release can be achieved at higher temperature, lower $\mathrm{pH}$ values. While, under NIR irradiation $(1.22 \mathrm{~W}, 980 \mathrm{~nm})$, a light increase in release $(9.55 \%$, compare with the $3.27 \%$ with NIR irradiation) was detected, due 25 to the energy adsorption of water and shrinkage of the polymers within the channels.

It is deserved to note that, for an ideal thermo-responsive nanostructure targeted for remotely stimuli-triggered release, it is preferable to exhibit a minimal drug release under physiological 30 temperature $\left(37^{\circ} \mathrm{C}\right)$, which is also called "zero premature release"; while controlled release is available due to the slightly local heating upon exposure to those remote stimuli. To satisfy this requirement, thermo-responsive macromolecules selected here should possess a LCST slightly above the physiological 35 temperature $\left(39-45^{\circ} \mathrm{C}\right)$, in order to avoid the local overheating. Since the most commonly used thermo-responsive homopolymers, e.g. PNiPAAm and PNVCL, possess a LCST of ca. $32{ }^{\circ} \mathrm{C}$, increasing their LCST is therefore required for their in vivo application. One of the commonly used strategies is to 40 incorporate another hydrophilic co-monomer. ${ }^{104}$ That is why usually thermo-responsive copolymers are used, e.g. poly(NIPAAm-co-AAm) with LCST of $32-50{ }^{\circ} \mathrm{C} . .^{195}$
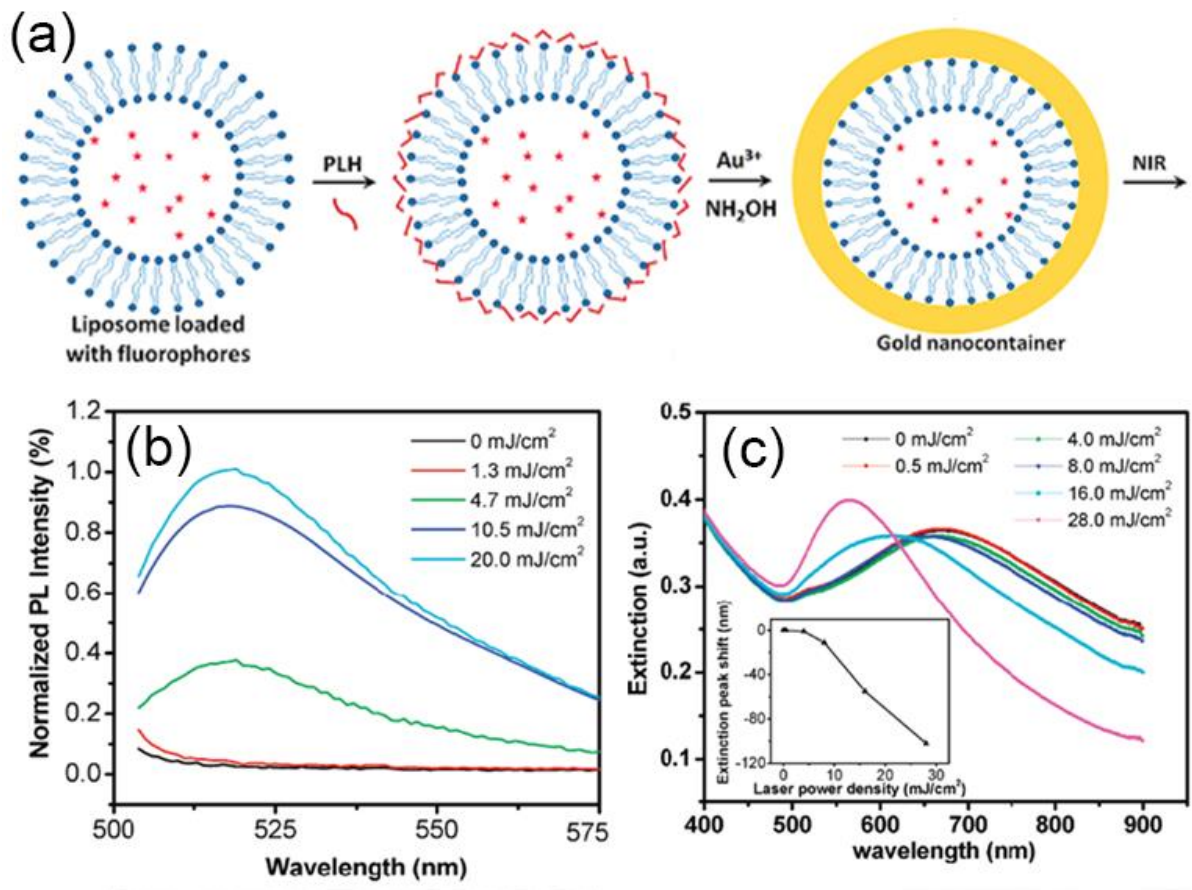
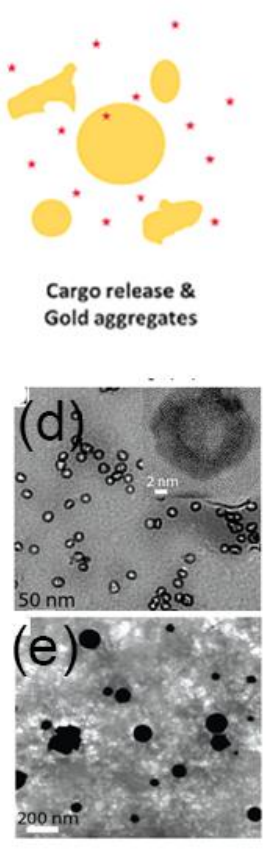

Fig. 17 Schematic illustration of liposome@gold nanovehicle and the underlying cargo release mechanism. Cargo (red stars) loaded liposomes were first coated with a layer of poly-L-histidine (PLH) for gold ion immobilization. Reduction with $\mathrm{NH}_{2} \mathrm{OH}$ led to the formation of the nanovehicles directed by the liposome scaffold. When irradiated with NIR light at their SPR, nanovehicles deformed and released the contents. (a) Triggered cargo release by pulsed laser irradiation, measured by fluorescence intensity changes. In the presence of an SPR matching laser, cargo release increased with increasing 50 laser power. (b) Nanovehicles deformation characterized by SPR spectral shift. The nanovehicles appeared stable when the laser irradiation was below 4 $\mathrm{mJ} / \mathrm{cm}^{2}$ but deformed under increasing laser power, which correlates well with the fluorescence measurements.(c) TEM and HRTEM (inset) images of gold nanovehicles (d), and TEM image of gold nanostructures after pulsed-laser irradiation at $28 \mathrm{~mJ} \mathrm{~cm}^{-2}$ (e). Adapted with permission from Ref. 212 . Copyright 2009 American Chemical Society.

\section{4. 6. Destruction of the nanostructures}

Triggered drug release can be achieved bv manv mechanisms. but it is quite challenging to place a device in vivo with drug release activated at will of the patient or a physician by an external trigger. Pressure-induced or thermo-induced rupture of ${ }_{60}$ nanovehicles is also one of the most reliable techniques to release the cargo molecules in a burst mode. As discussed previously, 


\section{Cite this: DOI: 10.1039/c0xx00000x}

\section{www.rsc.org/xxxxxx}

ARTICLE TYPE

triggered release from liposomes could be achieved with ultrasound, which causes either localized heating and/or structural deformation. ${ }^{26}$ These two effects contribute to triggered release based on the mechanism of enhanced diffusion and/or phase5 change of the TSLs lipid membrane. Moreover, when higherenergy ultrasound is used and structure deformation exceeds the elastic limitation of the lipid membrane, mechanical disruption of the liposomal structure occurs, leading to a burst release. ${ }^{45,239}$

As far as we know, the NIR-triggered structure disintegration 10 of those nanovehicles is mainly dealing with the nanostructures based on combination of GNPs and liposomes, such as GNPs adsorbed on liposomes, gold nanoshells over the liposomes or embedding GNPs within liposomes. Similarly to other gold-based nanostructures, spontaneous local heating could also be generated 15 under NIR irradiation for those liposome@GNPs nanostructures. The local temperatures were reported to be able to reach few hundreds of degrees, depending on the irradiation conditions. ${ }^{150}$, 201 This high-efficient photothermal conversion induces significant thermal and mechanical stress, or even destabilizes the
20 nanostructures, resulting in the rupture of nanovehicles and subsequent cargo release. In a representative work by Gao and coll., ${ }^{212}$ liposome@gold nanostructure was reported for spatially and temporally triggered drug release (Fig. 17a). As a proof of concept, this kind of nanovehicle was loaded with cargo 25 molecules of organic dye carboxyfluorescein or DOX, and a leakage-free character was evidenced over extended storage periods. However, the cargoes could be also efficiently released under NIR laser irradiation (Fig. 17b). After NIR irradiation, the nanostructure extinction profiles exhibited a significant blue-shift

30 (Fig. 17c). Based on TEM observation, after laser illumination, the majority of nanovehicles were converted to large gold aggregates (Fig. 17e), compared with the hollow gold nanoshell structure before irradiation (Fig. 17d). All of these results corroborated the NIR-induced disintegration of the 35 nanostructures. Similar works based on liposome@gold nanostructures were also reported by $\mathrm{Wu}$ and coll. ${ }^{150}$, Srivastava and coll. ${ }^{206}$, De and coll., ${ }^{205}$ for the NIR-triggered release with the underlying disintegration mechanism.
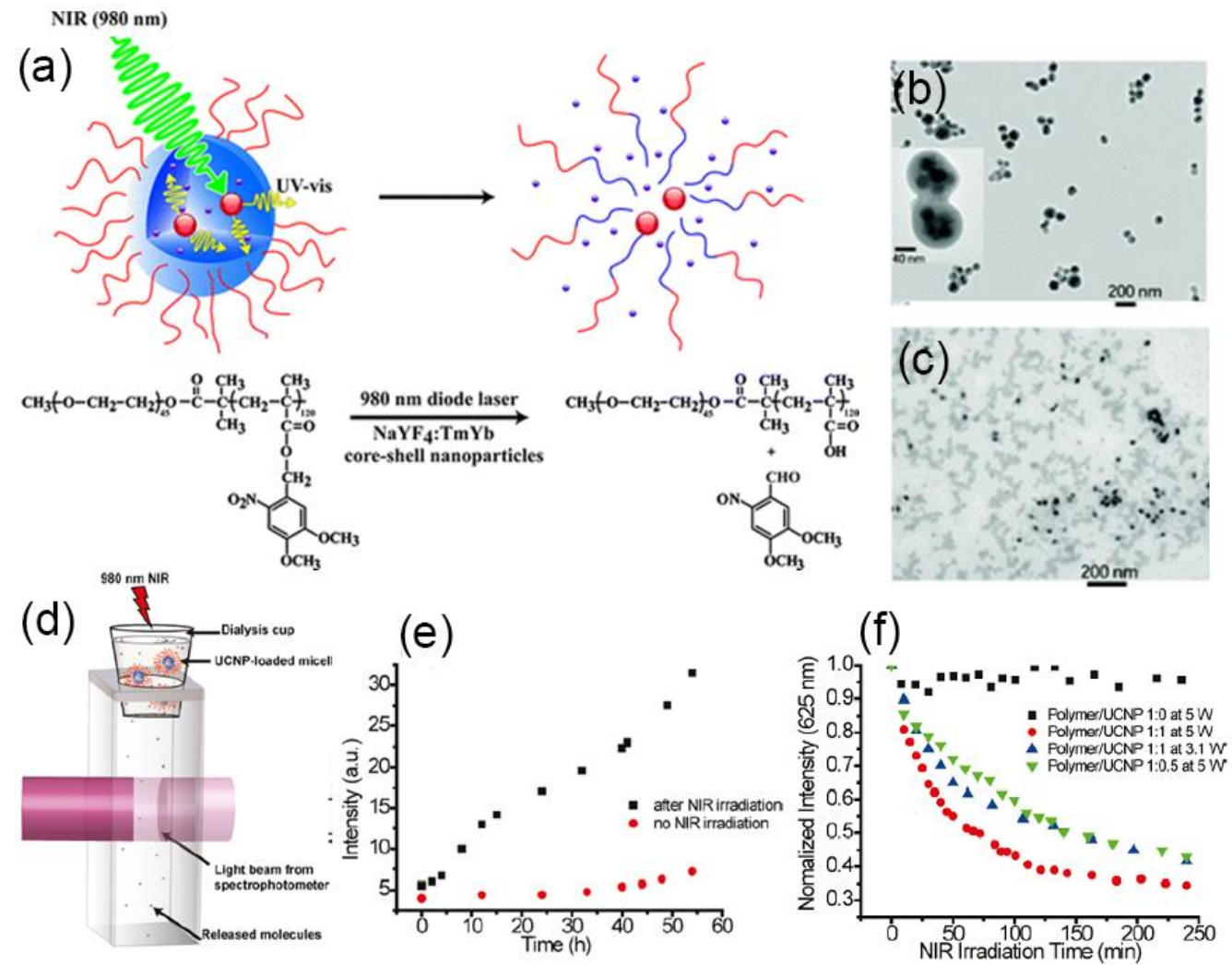

40

Fig. 18 Schematic illustration of using NIR light excitation of up-converting nanoparticles to trigger dissociation of micelles, and NIR light-triggered photoreaction with the used $o$-nitrobenzyl moieties and $\mathrm{NaYF}_{4}$ : TmYb nanoparticles (a). TEM image of UCNP-loaded BCP micelles before (b) and after (c) NIR light irradiation; Schematic illustration of setup used to detect species diffusing from a BCP micellar solution through a dialysis membrane into aqueous solution underneath under NIR irradiation (d). Evolution of the normalized fluorescence intensity of NR over time during the NIR irradiation (e); 45 and plotting of normalized fluorescence intensity of NR vs. NIR irradiation (polymer/UCNP is the weight ratio used in the preparation of UCNP-loaded BCP micelles). Adapted with permission from Ref. 116. Copyright 2011 American Chemical Society. 
Apart from the gold nanoshells, silica or liposomal nanostructures, NIR-triggered disintegration of self-assembled micelle nanostructures is another simple and efficient way for 5 remotely triggered release. ${ }^{240}$ As mentioned above, with the help of UCNPs, photo-isomerizable moieties, e.g. azobenzene groups, can be used for remotely triggered release based on the mechanism of light-induced trans-cis transition. On the other hand, photo-labile moieties, such as benzoin and $o$-nitrobenzyl 10 derivatives, can also be adopted for NIR-triggered release based on the light-induced disintegration of nanostructures. Branda and coll. ${ }^{116}$ reported micellar nanostructures made of an amphiphilic copolymer bearing $o$-nitrobenzyl moieties, in which Nile Red and UCNPs were loaded (Fig. 18a-b). The release mechanism is 15 based on the photo-induced cleavage of $o$-nitrobenzyl groups by UV light, which is emitted by the UCNPs upon NIR irradiation $(980 \mathrm{~nm}, 5 \mathrm{~W}, 4 \mathrm{~h}$, Fig. 18d), followed by the subsequent hydrophobic/hydrophilic transition, disintegration of micelles and triggered drug release. The same strategy was also extended in 20 their subsequent work ${ }^{115}$ on reversibly cross-linked hydrogels, which were cross-linked with molecules bearing $o$-nitrobenzyl moieties, and developed for DDS. NIR-induced gel-sol transition was evidenced with the release of trapped cargoes due to the decross-linking (Fig. 18c, and 18e-f). Gu and coll. ${ }^{117}$ as well as 25 Almutairi and coll. ${ }^{118}$ also reported polymer micelles, made of $o$ nitrobenzyl-bearing amphiphilic block copolymers, with UCNPsand drug encapsulated. NIR illumination could also lead to the dissociation of the micelles, accompanied with a burst release of pre-loaded hydrophobic cargoes.

30 Similarly to the NIR-induced structural disintegration, AMFtriggered release based on the same strategy was also engaged with thermo-labile nanostructures, such as gold nanoshells, ${ }^{213}$ combination of magnetic nanoparticles with liposomes, ${ }^{44}$ or silica nanoshells. ${ }^{241,242}$

\section{Conclusion, prospective and challenges}

DDS responding to local stimuli, such as variation in $\mathrm{pH}$ or temperature, activation of reductive agent or enzyme, could effectively provide desirable release, even in a passive mode, but 40 fail in diseases treatment following the biological rhythms. ${ }^{10}$ However, it can be undoubtedly concluded that nanovehicles aimed for remotely triggered release could offer an alternative solution. Moreover, remote triggers of these systems present various advantages, such as the improved efficiency, good spatial 45 and temporal controllability over the release, lack of undesirable adverse effect, minimal release during the in vivo delivery, etc. ${ }^{11}$ With the increasing requirements on clinical practices, it might demand delivering drugs at the right time, right place and also with right amounts, which holds good promises of benefit to the 50 patients, but presents huge challenge for remotely-triggered DDS. Further requirements, such as zero premature release, pulsatile release, targeting delivery and minimal heating up to the surroundings, have been brought up in some studies. In the following sections, we present a concise comment on each 55 character.

\section{1. Zero premature release}

From the clinical viewpoint, it is highly desirable to achieve a zero or nearly-zero premature release before delivering the drug${ }_{60}$ loaded nanovehicles to the disease sites, in order to improve the therapeutic efficiency and avoid serious side effects in the case of the delivery of highly toxic guest molecules. Usually, the diffusion of guest molecules from the nanovehicles into the surrounding environment is an unavoidable thermodynamic 65 procedure under a concentration gradient. Conventional DDS, such as self-assembled polymer micelles or liposomes, polymer nanogels, mesoporous silica, and stimuli-responsive inorganic/organic nanohybrids, cannot satisfy this requirement. That is why numerous attempts have been made, e.g. gating 70 systems in the mesoporous silica ${ }^{2}$ and reversibly-cross-linked micelles or nanogels, ${ }^{4}$ in order to obtain a good entrapment of the cargo molecules and get rid of the risk from undesired premature release. Once such a nanostructure is excited with specific stimuli right after targeting to the disease sites, the guest molecules are 75 released, resulting in an improved therapeutic efficacy and decreased deleterious side effects. Taking the advantageous merits of remote triggers, such as AMF, NIR and ultrasound, a better spatial and temporal control could be accomplished. However, the higher the barrier from the nanostructures to trap 80 the cargo molecules, the higher the energy needed to trigger the release, subsequently the higher the risk of adverse effect, such as local overheating. Thus, an optimal compromise between the trapping nanostructures and remote stimuli should be found before the in vivo application.

85

\section{2. Pulsatile release}

For an ideal DDS, it is not only very important to achieve a "zero premature release" over prolonged period of in vivo delivery as required, but also for some special purposes it might 90 be necessary to release the pre-loaded guest molecules in small portions following a programmed mode, called "pulsatile release". ${ }^{243}$ Compared with the conventional burst or sustainable release, the pulsatile release might present lots of merits, such as reduced adverse effects and improved tolerability, limited risk of 95 overdose, predictable and reproducible release behaviours, etc. ${ }^{244}$ In the human body, many vital functions are regulated by transient release of bioactive substances at a specific time and site under physiological conditions. Thus, in order to mimic the function of living systems and in view of emerging 100 chronotherapeutic approaches, it is of necessity to consider this requirement. Normally, pulsatile DDS can be pre-programmed and self-regulated depending on the presence of some specific molecules or variation in physiological variables, or can be externally actuated by applying various external stimuli outside 105 the body. As for the former regulation, taking temperature as an example, thermo-responsive polymer-based DDS could release the cargoes in a pulsatile mode upon alternating heating/cooling treatment, due to the de-swelling/swelling conformation of the polymer chains. Similar structures for pulsatile release could be 110 found in the review by Okano and coll. ${ }^{244}$ and Park and coll. ${ }^{245}$

Alternative strategy should be the application of remote triggers, since they possess a spatial and temporal control over 


\section{Cite this: DOI: 10.1039/c0xx00000x}

\section{www.rsc.org/xxxxxx}

ARTICLE TYPE

the in vivo release behaviour. Furthermore, when using remote triggers for pulsatile release, it might also avoid the side effect caused by over exposure to AMF, NIR, ultrasound, such as normal tissue damage from local overheating. ${ }^{246}$ Up to now, 5 significant progress has been made, and a lot of trails have been done, such as the magnetically, ${ }^{19}, 182,246-249 \mathrm{NIR}^{86,88,179,183}$ and ultrasound triggered pulsatile release. ${ }^{198}$ In these works, they disclosed the possibility of switching on/off the drug release, and sustainable release could be obtained by more on/off cycles. Even 10 though, at this moment, this kind of DDS still bear some drawbacks even at the laboratory scale, such as lack of manufacturing reproducibility and efficacy, large number of process variables, limited drug loading, need of advanced technology and trained/skilled persons for manufacturing, ${ }^{243}$ we 15 still believe that their development is definitely a formidable challenge for nanomedicine.

\section{3. Mild or minimal warming of the surroundings}

Generally, hyperthermia is also categorized into local, 20 regional and whole body hyperthermia, depending on the location of disease and also heating strategies. A local hyperthermia involves the generation of heat only in a small area of interest, such as a tumour site. Regional hyperthermia refers the heating treatment within a larger area, such as a whole organ. The whole

25 body hyperthermia is usually applied when the cancer cells spread throughout the whole body. ${ }^{250,251}$ As known to us, at temperature of $41-45^{\circ} \mathrm{C}$, tumour cells begin to show signs of apoptosis, while higher temperature above $50^{\circ} \mathrm{C}$ is associated with less apoptosis but more frank necrosis, due to the thermo30 induced protein denaturation, revealing potential tumour treatment via hyperthermia. ${ }^{36}$ A concern to keep in mind for tumour treatment via hyperthermia, particularly those relying on remote triggers, is that patients rarely die of their primary tumours, since many primary tumours can be surgically removed. 35 Metastatic disease is a common cause of death in advanced cancer, but small metastatic cancers are not accessible via remote triggers. ${ }^{22}$ Hence, applications for remote triggered hyperthermia formulations should be carefully chosen, for reasons of preserving quality of life.

40 In addition to such direct hyperthermia treatments, these nanovehicles are explored to combine chemotherapeutic reagents and hyperthermia therapy or phototherapy. This combined strategy results in eliminating a part of the tumour cells, and also makes the resistant tumour cells more vulnerable to other 45 treatments, such as chemotherapeutic reagents. Based on this blueprint, local hyperthermia is gaining increasing attention due to controlled intracellular heating within specified sites. ${ }^{252}$ As discussed above, one of the underlying mechanisms for remotelytriggered release is to heat the nanovehicles in order to induce the 50 cleavage of covalent bonding, phase change, trans-cis transition, structural disintegration, etc. However, some details should also be taken into consideration over this strategy. Indeed on one hand, for the thermal cleavage of covalent bonds, e.g. Au-S, 220 Diels-Alder cycloadduct, ${ }^{69}$ and azo group, ${ }^{68}$ a high-energy trigger 55 is needed, and, on the other hand, overheating of the surrounding might result in epidermal detachment, burns, and necrosis of the viable epidermis or underlying tissues. Some other disadvantages, such as limited penetration of heat into body tissues and undesirable under-dosage in some target region might further 60 limit the clinical use. That is why recent studies, especially those conducted with higher-amplitude remote stimuli, have paid more attention to control and minimize thermal effects.

Generally, the in vivo therapeutic effect would be decided by a lot of variables. In a first approximation, the concentration of 65 the delivered drugs at the disease sites depends on the plasma concentration of the nanovehicles, the drug loading capacity, the release kinetic and the overall period of hyperthermia treatment. The aforementioned parameters define the requirements for the route of administration and the properties of nanovehicles.

${ }_{70}$ Furthermore, biological factors like tumour vascularization and perfusion are of utmost importance, as they modulate not only the drug uptake within the tumours but may also strongly vary between patients even for the same tumour type. ${ }^{22,}{ }^{251}$ From empirical interpretation, a relative high targeting amount, namely 75 higher targeting efficiency of the nanovehicles and/or higher loading capacity, might be in favor of using lower-amplitude remote triggers to reach the threshold of local heating. Optimal method to non-invasively maintain a minimal local heating, but with desirable release, would therefore be of high clinical value. 80

\section{4. Targeted delivery and bio-distribution}

The in vivo delivery of nanovehicles to the desirable sites is critical in both DDS and other biomedical uses, such as diagnostic imaging. In all cases, nonspecific cell binding can 85 place healthy tissue at risk. To limit non-specific binding, different nanostructures have been engineered with some targeting ligands, which show an affinity for specific tissues. Thus, they could accumulate within the desirable sites through an active approach. For magnetic nanovehicles, external magnetic 90 guidance could also contribute to a targeted delivery, ${ }^{253}$ but the required magnetic properties are very different from those needed for AMF-triggered release. Passive targeting takes advantage of the predetermined physicochemical properties of the nanovehicles, and specifically migrate to a given disease region. ${ }_{95}$ For example, targeting to solid tumour tissue can be achieved through the passive mechanism, i.e. EPR effect. ${ }^{23}$ However, EPR is limited to specific metastatic solid tumours, and successful implementation highly depends upon a number of factors including degree of capillary disorder, blood flow, and lymphatic 100 drainage rate, making the effective management more difficult.

Passive targeting is available for only certain in vivo applications and does not necessarily guarantee the internalization of nanovehicles by those specifically targeted cells. Nanovehicles, which are additionally marked with 
molecular targeting ligands, might show an active targeting character. ${ }^{254} \mathrm{~A}$ number of nanovehicles have been implemented with targeting ligands into their designs with various targeting moieties, e.g. peptides, antibody or folic acid, which are approved 5 by FDA for clinically treatment of malignant tumours. ${ }^{254}$ In an ideal targeting ligands/receptors pair, identification of these targeting ligands does not express on normal cells. Therefore, it allows targeting these nanovehicles to only malignant cells for further chemotherapeutic and/or hyperthermia treatment, 10 promising minimal therapy-related toxicities. Unfortunately, in those experimental practices dealing with targeted delivery, most potential cancer targeting ligands, e.g. epithelial growth factor receptors, can always over-express in cancer cells but not uniquely. ${ }^{254}$ Constitutive expression of ligands on normal cells 15 leads to uptaking targeted nanovehicles in nonmalignant tissues, leading to potential toxicities and limitations for drug delivery or hyperthermia. Any possible targeting moieties for cancer cells may provide a feasible means for targeting delivery, but require careful evaluations over the targeting efficacy.

20 Experts have forecasted a continuously rising demand for dosage from such hybrid nanovehicles with the help of remote triggers. Thus, more and more attempts are being made to design and fabricate various DDS according to patient requirements, in terms of therapeutic efficacy and compliance. Remotely-triggered 25 release formulations are smart DDS specially suited to satisfy these needs, and offer interesting options for intelligent management over the disease treatments. We are convinced that with increase in technological advancement and better design parameters, these systems will be optimized step by step in the 30 near future and reach translational developments towards patients.

\section{Acknowledgements}

The authors thank the Belgian National Funds for Scientific ${ }_{35}$ Research (F.R.S.-FNRS), the European Community in the frame of the Erasmus Mundus International doctoral school IDSFunMat and the Science Policy Office of the Belgian Federal Government (PAI VII-05) for their financial support. C.D. is Research Director by F.R.S.-FNRS.

40

\section{Notes and references}

a Centre for Education and Research on Macromolecules (CERM), University of Liege, Chemistry Department, B6A Sart Tilman, B-4000 Liège, Belgium. Fax: +32 4366 3497; Tel: +3243663565 45 c.jerome@ulg.ac.be

b CNRS, Univ. Bordeaux, ICMCB, UPR 9048, F-33600 Pessac, France duguet@icmcb.u-bordeauxl.fr, Fax: +33 540002 761,Tel: +33 540002 651

†Current address: Melville Laboratory for Polymer Synthesis, ${ }_{50}$ Department of Chemistry, University of Cambridge, Cambridge, CB2 $1 E W$, United Kingdom

\section{References}

1. N. Rapoport, Prog. Polym. Sci., 2007, 32, 962-990.

55 2. C. Coll, A. Bernardos, R. Martinez-Manez and F. Sancenon, Acc. Chem. Res., 2013, 46, 339-349.

3. H. Wei, R. X. Zhuo and X. Z. Zhang, Prog. Polym. Sci., 2012, 38, 503-535.
4. Y. Shao, W. Huang, C. Shi, S. T. Atkinson and J. Luo, Ther. Deliv., 2012, 3, 1409-1427.

60 5. D. R. Lynch and S. Snyder, Annu. Rev. Biochem., 1986, 55, 773-799.

6. S. Ganta, H. Devalapally, A. Shahiwala and M. Amiji, J. Control. Release, 2008, 126, 187-204.

7. A. Balaceanu, V. Mayorga, W. J. Lin, M. P. Schurings, D. E. Demco, A. Boker, M. A. Winnik and A. Pich, Colloid Polym. Sci., 2013, 291, 21-31.

658 . M. A. Stuart, W. T. Huck, J. Genzer, M. Muller, C. Ober, M. Stamm, G. B. Sukhorukov, I. Szleifer, V. V. Tsukruk, M. Urban, F. Winnik, S. Zauscher, I. Luzinov and S. Minko, Nat. Mater., 2010, 9, 101-113.

9. B. P. Timko and D. S. Kohane, Expert Opin. Drug Deliv., 2014, 11, 16811685.

70 10. B. P. Timko, K. Whitehead, W. W. Gao, D. S. Kohane, O. Farokhzad, D. Anderson and R. Langer, Annu. Rev. Mater. Res., 2011, 41, 1-20.

11. A. Chan, R. P. Orme, R. A. Fricker and P. Roach, Adv. Drug Deliv. Rev., 2013, 65, 497-514.

12. C. S. Brazel, Pharm. Res., 2009, 26, 644-656.

75 13. J. Thevenot, H. Oliveira, O. Sandre and S. Lecommandoux, Chem. Soc. Rev., 2013. 42, 512-529.

14. T. Y. Liu, S. H. Hu, D. M. Liu, S. Y. Chen and I. W. Chen, Nano Today, $2009,4,52-65$.

15. S. Lal, S. E. Clare and N. J. Halas, Acc. Chem. Res., 2008, 41, 1842-1851.

80 16. A. M. Alkilany, S. E. Lohse and C. J. Murphy, Acc. Chem. Res., 2012, 46, 650-661.

17. A. Schroeder, J. Kost and Y. Barenholz, Chem. Phys. Lipids, 2009, 162, 116.

18. Y. Z. Zhao, L. N. Du, C. T. Lu, Y. G. Jin and S. P. Ge, Int. J. Nanomedicine, 2013, 8, 1621-1633.

19. T. Y. Liu, M. Y. Wu, M. H. Lin and F. Y. Yang, Acta Biomater., 2013, 9, 5453-5463.

20. B. P. Timko, T. Dvir and D. S. Kohane, Adv. Mater., 2010, 22, 4925-4943.

21. A. Rösler, G. W. Vandermeulen and H.-A. Klok, Adv. Drug Deliv. Rev., $90 \quad 2012, \mathbf{6 4}, 270-279$.

22. I. Brigger, C. Dubernet and P. Couvreur, Adv. Drug Deliv. Rev., 2012, 64, 24-36.

23.H. Maeda, J. Wu, T. Sawa, Y. Matsumura and K. Hori, J. Control. Release, 2000, 65, 271-284.

95 24. S. Mornet, S. Vasseur, F. Grasset, P. Veverka, G. Goglio, A. Demourgues, J. Portier, E. Pollert and E. Duguet, Prog. Solid State Chem., 2006, 34, 237-247.

25. Y. Xia, W. Li, C. M. Cobley, J. Chen, X. Xia, Q. Zhang, M. Yang, E. C. Cho and P. K. Brown, Acc. Chem. Res., 2011, 44, 914-924.

100 26. S. Bibi, E. Lattmann, A. R. Mohammed and Y. Perrie, J. Microencapsul., 2012, 29, 262-276.

27.H. Fattahi, S. Laurent, F. Liu, N. Arsalani, L. Vander Elst and R. N. Muller, Nanomedicine, 2011, 6, 529-544.

28.C. Deng, Y. Jiang, R. Cheng, F. Meng and Z. Zhong, Nano Today, 2012, 7, $105 \quad 467-480$.

29. J. H. Ryu, H. Koo, I. C. Sun, S. H. Yuk, K. Choi, K. Kim and I. C. Kwon, Adv. Drug Deliv. Rev., 2012, 64, 1447-1458.

30. B. Haley and E. Frenkel, in Urologic Oncology: Seminars and original investigations, Elsevier, 2008, 57-64.

110 31. S. Laurent, D. Forge, M. Port, A. Roch, C. Robic, L. Vander Elst and R. N. Muller, Chem. Rev., 2008, 108, 2064-2110.

32. N. Z. Knezevic, E. Ruiz-Hernandez, W. E. Hennink and M. Vallet-Regi, RSC Adv., 2013, 3, 9584-9593.

33. B. P. Timko and D. S. Kohane, Clin. Ther., 2012, 34, S25-35.

115 34. N. Fomina, J. Sankaranarayanan and A. Almutairi, Adv. Drug Deliv. Rev., 2012, 64, 1005-1020.

35. S. Mura, J. Nicolas and P. Couvreur, Nat. Mater., 2013, 12, 991-1003.

36. S. Mornet, S. Vasseur, F. Grasset and E. Duguet, J. Mater. Chem., 2004, 14, 2161-2175.

120 37. A. Hervault and N. T. K. Thanh, Nanoscale, 2014, 6, 11553-11573.

38. N. Fomina, J. Sankaranarayanan and A. Almutairi, Adv. Drug Deliv. Rev., 2012, 64, 1005-1020.

39. M. Treguer-Delapierre, J. Majimel, S. Mornet, E. Duguet and S. Ravaine, Gold Bull., 2008, 41, 195-207. 


\section{Cite this: DOI: 10.1039/c0xx00000x}

\section{www.rsc.org/xxxxxx}

ARTICLE TYPE

40. P. Rai, S. Mallidi, X. Zheng, R. Rahmanzadeh, Y. Mir, S. Elrington, A. Khurshid and T. Hasan, Adv. Drug Deliv. Rev., 2010, 62, 1094-1124.

41. S. Lal, S. E. Clare and N. J. Halas, Acc. Chem. Res., 2008, 41, 1842-1851.

42. C. M. Cobley, L. Au, J. Chen and Y. Xia, Expert Opin. Drug Deliv., 2010, 7, 577-587.

43. B. Geers, H. Dewitte, S. C. De Smedt and I. Lentacker, J. Control. Release, 2012, 164, 248-255.

44. P. M. Peiris, L. Bauer, R. Toy, E. Tran, J. Pansky, E. Doolittle, E. Schmidt, E. Hayden, A. Mayer and R. A. Keri, ACS Nano, 2012, 6, 4157-4168.

10 45. M. Raoof, B. T. Cisneros, A. Guven, S. Phounsavath, S. J. Corr, L. J. Wilson and S. A. Curley, Biomaterials, 2013, 34, 1862-1869.

46. S. Mo, C. C. Coussios, L. Seymour and R. Carlisle, Expert Opin. Drug Deliv., 2012, 9, 1525-1538.

47. E. Oude Blenke, E. Mastrobattista and R. M. Schiffelers, Expert Opin. Drug Deliv., 2013, 10, 1399-1410.

48. A. M. Alkilany, L. B. Thompson, S. P. Boulos, P. N. Sisco and C. J. Murphy, Adv. Drug Deliv. Rev., 2012, 64, 190-199.

49. P. K. Jain, W. Qian and M. A. El-Sayed, J. Am. Chem. Soc., 2006, 128, 2426-2433.

20 50. T. Y. Liu, S. H. Hu, D. M. Liu and S. Y. Chen, Langmuir, 2006, 22, $5974-$ 5978.

51. G. B. Braun, A. Pallaoro, G. Wu, D. Missirlis, J. A. Zasadzinski, M. Tirrell and N. O. Reich, ACS Nano, 2009, 3, 2007-2015.

52. A. Wijaya, S. B. Schaffer, I. G. Pallares and K. Hamad-Schifferli, ACS

25 Nano, 2009, 3, 80-86.

53. H. Takahashi, Y. Niidome and S. Yamada, Chem. Commun., 2005, 22472249.

54. S. Yamshita, Y. Niidome, Y. Katayama and T. Niidome, Chem. Lett., 2009, 38, 226-227.

30 55. S. S. Jang, Y. H. Jang, Y. H. Kim, W. A. Goddard, A. H. Flood, B. W. Laursen, H. R. Tseng, J. F. Stoddart, J. O. Jeppesen, J. W. Choi, D. W. Steuerman, E. Deionno and J. R. Heath, J. Am. Chem. Soc., 2005, 127, 1563-1575.

56. R. Huschka, J. Zuloaga, M. W. Knight, L. V. Brown, P. Nordlander and N.

35 J. Halas, J. Am. Chem. Soc., 2011, 133, 12247-12255.

57. M. Chen, P. Qiu, X. He, K. Wang, S. Chen, S. Yang and X. Ye, J. Mater. Chem. B, 2014, 2, 3204-3213.

58. X. Huang, A. Pallaoro, G. B. Braun, D. P. Morales, M. O. Ogunyankin, J. Zasadzinski and N. O. Reich, Nano Lett., 2014, 14, 2046-2051.

40 59. Y. Tao, E. Ju, Z. Liu, K. Dong, J. Ren and X. Qu, Biomaterials, 2014, 35, 6646-6656.

60. A. Barhoumi, R. Huschka, R. Bardhan, M. W. Knight and N. J. Halas, Chem. Phy. Lett., 2009, 482, 171-179.

61. R. Huschka, O. Neumann, A. Barhoumi and N. J. Halas, Nano Lett., 2010, 10, 4117-4122.

62. Y. Horiguchi, S. Yamshita, T. Niidome, N. Nakashima and Y. Niidome, Chem. Lett., 2008, 37, 718-719.

63. S. E. Lee, G. L. Liu, F. Kim and L. P. Lee, Nano Lett., 2009, 9, 562-570.

64. S. Yamashita, H. Fukushima, Y. Akiyama, Y. Niidome, T. Mori, Y. Katayama and T. Niidome, Bioorg. Med. Chem., 2011, 19, 2130-2135.

65. W. Lu, G. Zhang, R. Zhang, L. G. Flores, 2nd, Q. Huang, J. G. Gelovani and C. Li, Cancer Res., 2010, 70, 3177-3188.

66. A. M. Derfus, G. von Maltzahn, T. J. Harris, T. Duza, K. S. Vecchio, E. Ruoslahti and S. N. Bhatia, Adv. Mater., 2007, 19, 3932-3936.

55 67. M. Banchelli, S. Nappini, C. Montis, M. Bonini, P. Canton, D. Berti and P. Baglioni, Phy. Chem. Chem. Phy., 2014, 16, 10023-10031.

68. A. Riedinger, P. Guardia, A. Curcio, M. A. Garcia, R. Cingolani, L. Manna and T. Pellegrino, Nano Lett., 2013, 13, 2399-2406.

69. S. Yamashita, H. Fukushima, Y. Niidome, T. Mori, Y. Katayama and T. 60 Niidome, Langmuir, 2011, 27, 14621-14626.
70. S. Ganta, H. Devalapally, A. Shahiwala and M. Amiji, J. Control. Release, 2008, 126, 187-204.

71. T. R. Kuo, V. A. Hovhannisyan, Y. C. Chao, S. L. Chao, S. J. Chiang, S. J. Lin, C. Y. Dong and C. C. Chen, J. Am. Chem. Soc., 2010, 132, 1416314171.

72. C. Feng, Y. Li, D. Yang, J. Hu, X. Zhang and X. Huang, Chem. Soc. Rev., 2011, 40, 1282-1295.

73. J. Liu, C. Detrembleur, A. Debuigne, M. C. De Pauw-Gillet, S. Mornet, L. Vander Elst, S. Laurent, C. Labrugère, E. Duguet and C. Jérôme, Nanoscale, 2013, 5, 11464-11477.

74. A. Debuigne, R. Poli, C. Jerome, R. Jerome and C. Detrembleur, Prog. Polym. Sci., 2009, 34, 211-239.

75. A. Debuigne, J. Warnant, R. Jérôme, I. Voets, A. de Keizer, M. A. Cohen Stuart and C. Detrembleur, Macromolecules, 2008, 41, 2353-2360.

75 76. S. Louguet, B. Rousseau, R. Epherre, N. Guidolin, G. Goglio, S. Mornet, E. Duguet, S. Lecommandoux and C. Schatz, Polym. Chem., 2012, 3, 14081417.

77. G. Sakellariou, D. Priftis and D. Baskaran, Chem. Soc. Rev., 2013, 42, 677-704.

80 78. G. Moad, E. Rizzardo and S. H. Thang, Polym. Int., 2010, 60, 9-25.

79. T. Kawano, Y. Niidome, T. Mori, Y. Katayama and T. Niidome, Bioconjug. Chem., 2009, 20, 209-212.

80. A. Aqil, H. J. Qiu, J. F. Greisch, R. Jerome, E. De Pauw and C. Jerome, Polymer, 2008, 49, 1145-1153.

85 81. S. Sistach, M. Beija, V. Rahal, A. Brulte, J. D. Marty, M. Destarac and C. Mingotaud, Chem.Mater., 2010, 22, 3712-3724.

82. M. Beija, E. Palleau, S. Sistach, X. G. Zhao, L. Ressier, C. Mingotaud, M. Destarac and J. D. Marty, J. Mater. Chem., 2012, 20, $9433-9442$.

83. M. Liang, I. C. Lin, M. R. Whittaker, R. F. Minchin, M. J. Monteiro and I. Toth, ACS Nano, 2010, 4, 403-413.

84. M. Beija, J. D. Marty and M. Destarac, Chem. Commun., 2011, 47, 28262828.

85. J. Liu, C. Detrembleur, M.-C. De Pauw-Gillet, S. Mornet, E. Duguet and C. Jérôme, Polym. Chem., 2014, 5, 799-813.

95 86. Y. N. Zhong, C. Yang, L. Cheng, F. H. Meng, Z. Y. Zhong and Z. Liu, Biomacromolecules, 2013, 14, 2411-2419.

87. Y. Zhong, C. Wang, R. Cheng, L. Cheng, F. Meng, Z. Liu and Z. Zhong, J. Control. Release, 2014, 195, 63-71.

88. J. Liu, C. Detrembleur, B. Grignard, M. C. De-Pauw-Gillet, S. Mornet, M. Treguer-Delapierre, Y. Petit, C. Jérôme and E. Duguet, Chem.Asian J, 2014, 9, 275-288.

89. E. Ju, Z. Li, Z. Liu, J. Ren and X. Qu, ACS Appl. Mater. Interfaces, 2014, 6, 4364-4370.

90. T. Chen, R. Ferris, J. M. Zhang, R. Ducker and S. Zauscher, Prog. Polym. 105 Sci., 2012, 35, 94-112.

91. W. Lv, S. Liu, X. Fan, S. Wang, G. Zhang and F. Zhang, Macromol. Rapid Commun., 2010, 31, 454-458.

92. R. Contreras-Caceres, A. Sanchez-Iglesias, M. Karg, I. Pastoriza-Santos, J. Perez-Juste, J. Pacifico, T. Hellweg, A. Fernandez-Barbero and L. M. LizMarzan, Adv. Mater., 2008, 20, 1666-1670.

93. Q. S. Wei, J. Jian and J. C. Shen, Macromol. Rapid Commun., 2008, 29, 645-650.

94. L. S. Zha, B. Banik and F. Alexis, Soft Matter, 2011, 7, 5908-5916.

95. N. S. Satarkar, D. Biswal and J. Z. Hilt, Soft Matter, 2010, 6, 2364-2371.

115 96. W. Wu, J. Shen, Z. Gai, K. Hong, P. Banerjee and S. Zhou, Biomaterials, 2011, 32, 9876-9887.

97. J. H. Kim and T. R. Lee, Chem. Mater., 2004, 16, 3647-3651.

98. R. A. Frimpong and J. Z. Hilt, Nanotechnology, 2008, 19, 175101.

99. R. Mastsuno, K. Yamamoto, H. Otsuka and A. Takahara, Macromolecules $120 \quad 2004,37,2203-2209$. 
100. W. H. Chiang, V. T. Ho, H. H. Chen, W. C. Huang, Y. F. Huang, S. C. Lin, C. S. Chern and H. C. Chiu, Langmuir, 2013, 29, 6434-6443.

101. H. Wang, J. Yi, S. Mukherjee, P. Banerjee and S. Zhou, Nanoscale, 2014, 6, 13001-13011.

5 102.K. Hayashi, M. Nakamura, H. Miki, S. Ozaki, M. Abe, T. Matsumoto, W. Sakamoto, T. Yogo and K. Ishimura, Theranostics, 2014, 4, 834-844.

103.H. Kang, A. C. Trondoli, G. Zhu, Y. Chen, Y. J. Chang, H. Liu, Y. F. Huang, X. Zhang and W. Tan, ACS Nano, 2011, 5, 5094-5099.

104.J. Liu, C. Detrembleur, A. Debuigne, M. C. De Pauw-Gillet, S. Mornet, L.

10 Vander Elst, S. Laurent, E. Duguet and C. Jérôme, J. Mater. Chem. B, 2014, 2, 1009-1023.

105.M. Hurtgen, C. Detrembleur, C. Jerome and A. Debuigne, Polym. Re.v, 2011, 51, 188-213.

106.M. Hurtgen, J. Liu, A. Debuigne, C. Jerome and C. Detrembleur, J. Polym.

15 Sci. Polym. Chem., 2012, 50, 400-408.

107.J. H. Gao, H. W. Gu and B. Xu, Acc. Chem. Res., 2009, 42, 1097-1107.

108.J. K. Oh and J. M. Park, Prog. Polym. Sci., 2011, 36, 168-189.

109.R. J. Hickey, A. S. Haynes, J. M. Kikkawa and S. J. Park, J. Am. Chem. Soc., 2011, 133, 1517-1525.

20 110.K. Lee, K. H. Bae, Y. Lee, S. H. Lee, C. H. Ahn and T. G. Park, Macromol. Biosci., 2010, 10, 239-245.

111.J. Yang, C. H. Lee, H. J. Ko, J. S. Suh, H. G. Yoon, K. Lee, Y. M. Huh and S. Haam, Angew. Chem. Int. Ed., 2007, 46, 8836-8839.

112.S. Chen, C. Guo, G. H. Hu, J. Wang, J. H. Ma, X. F. Liang, L. Zheng and

25 H. Z. Liu, Langmuir, 2006, 22, 9704-9711.

113.T. Azzam and A. Eisenberg, Langmuir, 2007, 23, 2126-2132.

114.Y. Kang and T. A. Taton, Angew. Chem. Int. Ed. Engl, 2005, 44, 409-412.

115.B. Yan, J. C. Boyer, D. Habault, N. R. Branda and Y. Zhao, J. Am. Chem. Soc., 2012, 134, 16558-16561.

30 116.B. Yan, J. C. Boyer, N. R. Branda and Y. Zhao, J. Am. Chem. Soc., 2011, 133, 19714-19717.

117.J. Cao, S. Huang, Y. Chen, S. Li, X. Li, D. Deng, Z. Qian, L. Tang and Y. Gu, Biomaterials, 2013, 34, 6272-6283.

118.M. L. Viger, M. Grossman, N. Fomina and A. Almutairi, Adv. Mater., $35 \quad 2013,25,3733-3738$.

119.M. Elsabahy and K. L. Wooley, Chem. Soc. Rev., 2012, 41, 2545-2561.

120.R. K. O'Reilly, C. J. Hawker and K. L. Wooley, Chem. Soc. Rev., 2006, 35, 1068-1083.

121.E. S. Read and S. P. Armes, Chem. Commun., 2007, 3021-3035.

40 122.T. Y. Liu, K. H. Liu, D. M. Liu, S. Y. Chen and I. W. Chen, Adv. Funct. Mater., 2009, 19, 616-623.

123.T. M. Allen and P. R. Cullis, Adv. Drug Deliv. Rev., 2013, 65, 36-48.

124.W. J. Mulder, G. J. Strijkers, G. A. van Tilborg, A. W. Griffioen and K. Nicolay, NMR Biomed., 2006, 19, 142-164.

45 125.D. C. Drummond, C. O. Noble, M. E. Hayes, J. W. Park and D. B. Kirpotin, J. Pharm. Sci., 2008, 97, 4696-4740.

126.M. B. Yatvin, J. N. Weinstein, W. H. Dennis and R. Blumenthal, Science, 1978, 202, 1290-1293.

127.J. N. Weinstein, R. L. Magin, M. B. Yatvin and D. S. Zaharko, Science,

$50 \quad 1979,204,188-191$.

128.E. Oude Blenke, E. Mastrobattista and R. M. Schiffelers, Expert Opin. Drug Deliv., 2013, 10, 1399-1410 .

129.H. Grull and S. Langereis, J. Control. Release, 2012, 161, 317-327.

130.Y. Ueno, S. Sonoda, R. Suzuki, M. Yokouchi, Y. Kawasoe, K. Tachibana,

55 K. Maruyama, T. Sakamoto and S. Komiya, Cancer Biol. Ther., 2011, 12, 270-277.

131.J. H. Ryu, H. Koo, I. C. Sun, S. H. Yuk, K. Choi, K. Kim and I. C. Kwon, Adv. Drug Deliv. Rev., 2012, 64, 1447-1458.

132.P. M. Peiris, L. Bauer, R. Toy, E. Tran, J. Pansky, E. Doolittle, E. Schmidt,

60 E. Hayden, A. Mayer, R. A. Keri, M. A. Griswold and E. Karathanasis, ACS Nano, 2012, 6, 4157-4168.

133.W. Su, H. Wang, S. Wang, Z. Liao, S. Kang, Y. Peng, L. Han and J. Chang, Int. J. Pharm., 2012, 426, 170-181.

134.M. de Smet, E. Heijman, S. Langereis, N. M. Hijnen and H. Grull, $J$.

65 Control. Release, 2011, 150, 102-110.
135.P. Pradhan, J. Giri, F. Rieken, C. Koch, O. Mykhaylyk, M. Doblinger, R. Banerjee, D. Bahadur and C. Plank, J. Control. Release, 2010, 142, 108121.

136.H. Zhang, H. Xia, J. Wang and Y. Li, J. Control. Release, 2009, 139, 3139.

137.K. Katagiri, Y. Imai, K. Koumoto, T. Kaiden, K. Kono and S. Aoshima, Small, 2011, 7, 1683-1689.

138.Y. Chen, A. Bose and G. D. Bothun, ACS Nano, 2010, 4, 3215-3221.

139.E. Amstad, J. Kohlbrecher, E. Muller, T. Schweizer, M. Textor and E.

75 Reimhult, Nano Lett., 2011, 11, 1664-1670.

140.R. Di Corato, G. Bealle, J. Kolosnjaj-Tabi, A. Espinosa, O. Clément, A. K. A. Silva, C. Menager and C. Wilhelm, ACS Nano, 2015, 9, 2904-2916.

141.H. Guo, W. Chen, X. Sun, Y.-N. Liu, J. Li and J. Wang, Carbohydrate Polym., 2015, 118, 209-217.

80 142.N. Lozano, W. T. Al-Jamal, A. Taruttis, N. Beziere, N. C. Burton, J. Van den Bossche, M. Mazza, E. Herzog, V. Ntziachristos and K. Kostarelos, J. Am. Chem. Soc., 2012, 134, 13256-13258.

143.A. Agarwal, M. A. Mackey, M. A. El-Sayed and R. V. Bellamkonda, ACS Nano, 2011, 5, 4919-4926.

85 144.D. V. Volodkin, A. G. Skirtach and H. Mohwald, Angew. Chem. Int. Ed., 2009, 48, 1807-1809.

145.T. S. Troutman, S. J. Leung and M. Romanowski, Adv. Mater., 2009, 21, 2334-2338.

146.L. Paasonen, T. Sipila, A. Subrizi, P. Laurinmaki, S. J. Butcher, M.

90 Rappolt, A. Yaghmur, A. Urtti and M. Yliperttula, J. Control. Release, 2010, 147, 136-143.

147.E. Y. Lukianova-Hleb, A. Belyanin, S. Kashinath, X. Wu and D. O. Lapotko, Biomaterials, 2012, 33, 1821-1826.

148.S. J. Leung and M. Romanowski, ACS Nano, 2012, 6, 9383-9391.

95 149.A. Kyrsting, P. M. Bendix, D. G. Stamou and L. B. Oddershede, Nano Lett., 2011, 11, 888-892.

150.G. Wu, A. Mikhailovsky, H. A. Khant, C. Fu, W. Chiu and J. A. Zasadzinski, J. Am. Chem. Soc., 2008, 130, 8175-8177.

151.C. Wu, C. Yu and M. Chu, Int. J. Nanomedicine, 2011, 6, 807-813.

100 152.Z. S. Al-Ahmady, W. T. Al-Jamal, J. V. Bossche, T. T. Bui, A. F. Drake, A. J. Mason and K. Kostarelos, ACS Nano, 2012, 6, 9335-9346.

153.G. Qin, Z. Li, R. Xia, F. Li, B. E. O'Neill, J. T. Goodwin, H. A. Khant, W. Chiu and K. C. Li, Nanotechnology, 2011, 22, 155605-155611.

154.I. I. Slowing, B. G. Trewyn, S. Giri and V. S. Y. Lin, Adv. Funct. Mater., 2007, 17, 1225-1236.

155.M. Vallet-Regi, M. Colilla and B. Gonzalez, Chem. Soc. Rev., 2011, 40, 596-607.

156.Y. Zhao, X. Sun, G. Zhang, B. G. Trewyn, Slowing, II and V. S. Lin, ACS Nano, 2011, 5, 1366-1375.

110 157.P. Yang, S. Gai and J. Lin, Chem. Soc. Rev., 2012, 41, 3679-3698.

158.I. I. Slowing, J. L. Vivero-Escoto, C. W. Wu and V. S. Y. Lin, Adv. Drug Deliv. Rev., 2008, 60, 1278-1288.

159.M. W. Ambrogio, C. R. Thomas, Y. L. Zhao, J. I. Zink and J. F. Stoddart, Acc. Chem. Res., 2011, 44, 903-913.

115 160.D. Tarn, C. E. Ashley, M. Xue, E. C. Carnes, J. I. Zink and C. J. Brinker, Acc. Chem. Res., 2013, 46, 792-801.

161.M. N. Antipina and G. B. Sukhorukov, Adv. Drug Deliv. Rev., 2011, 63, 716-729.

162.Y. T. Chang, P. Y. Liao, H. S. Sheu, Y. J. Tseng, F. Y. Cheng and C. S. 120 Yeh, Adv. Mater., 2012, 24, 3309-3314.

163.X. Yang, X. Liu, Z. Liu, F. Pu, J. Ren and X. Qu, Adv. Mater., 2012, 24, 2890-2895.

164. Z. Zhang, L. Wang, J. Wang, X. Jiang, X. Li, Z. Hu, Y. Ji, X. Wu and C. Chen, Adv. Mater., 2012, 24, 1418-1423.

125 165.S. Shen, H. Tang, X. Zhang, J. Ren, Z. Pang, D. Wang, H. Gao, Y. Qian, X. Jiang and W. Yang, Biomaterials, 2013, 34, 3150-3158.

166.X. Yang, Z. Liu, Z. Li, F. Pu, J. Ren and X. Qu, Chem. Eur. J., 2013, 19, 10388-10394.

167.J. Liu, C. Detrembleur, M. Hurtgen, A. Debuigne, M.-C. De Pauw-Gillet,

130 S. Mornet, E. Duguet and C. Jérôme, Polym. Chem., 2014, 5, 77-88.

168.J. Liu, W. Bu, L. Pan and J. Shi, Angew. Chem. Int. Ed., 2013, 52, 43754379. 


\section{Cite this: DOI: 10.1039/c0xx00000x}

\section{www.rsc.org/xxxxxx}

ARTICLE TYPE

169.M. K. Jayakumar, N. M. Idris and Y. Zhang, Proc. Natl. Acad. Sci., 2012, 109, 8483-8488.

170.X. Zhang, P. P. Yang, Y. L. Dai, P. A. Ma, X. J. Li, Z. Y. Hou, X. J. Kang, C. X. Li and J. Lin, Adv. Funct. Mater., 2013, 23, 4067-4078.

5 171.A. Baeza, E. Guisasola, E. Ruiz-Hernandez and M. Vallet-Regi, Chem. Mater., 2012, 24, 517-524.

172.S. H. Hu, T. Y. Liu, H. Y. Huang, D. M. Liu and S. Y. Chen, Langmuir, 2008, 24, 239-244.

173.W. P. Li, P. Y. Liao, C. H. Su and C. S. Yeh, J. Am. Chem. Soc., 2014, 136, 10062-10075.

174.G. Béalle, R. Di Corato, J. Kolosnjaj-Tabi, V. Dupuis, O. Clément, F. Gazeau, C. Wilhelm, C. Ménager, Langmuir, 2012, 28, 11834-11842.

175.C. R. Thomas, D. P. Ferris, J. H. Lee, E. Choi, M. H. Cho, E. S. Kim, J. F. Stoddart, J. S. Shin, J. Cheon and J. I. Zink, J. Am. Chem. Soc., 2010, 132,

$15 \quad 10623-10625$.

176.E. Ruiz-Hernandez, A. Baeza and M. Vallet-Regi, ACS Nano, 2011, 5, 1259-1266.

177.S. Giri, B. G. Trewyn, M. P. Stellmaker and V. S. Lin, Angew. Chem. Int. Ed., 2005, 44, 5038-5044.

20 178.P. J. Chen, S. H. Hu, C. S. Hsiao, Y. Y. Chen, D. M. Liu and S. Y. Chen, J. Mater. Chem., 2007, 21, 2535-2543.

179.E. Aznar, M. D. Marcos, R. Martinez-Manez, F. Sancenon, J. Soto, P. Amoros and C. Guillem, J. Am. Chem. Soc., 2009, 131, 6833-6843.

180.J. Yang, D. Shen, L. Zhou, W. Li, X. Li, C. Yao, R. Wang, A. M. El-Toni, F. Zhang and D. Zhao, Chem. Mater., 2013, 25, 3030-3037.

181.K. Khaletskaya, J. Reboul, M. Meilikhov, M. Nakahama, S. Diring, M. Tsujimoto, S. Isoda, F. Kim, K. Kamei, R. A. Fischer, S. Kitagawa and S. Furukawa, J. Am. Chem. Soc., 2013, 135, 10998-11005.

182.J. Liu, C. Detrembleur, M. C. De Pauw-Gillet, S. Mornet, L. Vander Elst,

30 S. Laurent, C. Jérôme and E. Duguet, J. Mater. Chem. B, 2014, 2, 59-70.

183.J. Liu, C. Detrembleur, M. C. De Pauw-Gillet, S. Mornet, C. Jérôme and E. Duguet, Small, 2015, 11, 2323-2332.

184.E. Amstad and E. Reimhult, Nanomedicine, 2012, 7, 145-164.

185.L. Li, Y. Guan, H. Liu, N. Hao, T. Liu, X. Meng, C. Fu, Y. Li, Q. Qu, Y.

35 Zhang, S. Ji, L. Chen, D. Chen and F. Tang, ACS Nano, 2011, 5, 74627470.

186.L. Li, F. Tang, H. Liu, T. Liu, N. Hao, D. Chen, X. Teng and J. He, ACS Nano, 2010, 4, 6874-6882.

187.F. Tang, L. Li and D. Chen, Adv. Mater., 2012, 24, 1504-1534.

40 188.H. Liu, D. Chen, L. Li, T. Liu, L. Tan, X. Wu and F. Tang, Angew. Chem. Int. Ed., 2011, 50, 891-895.

189.H. Liu, T. Liu, L. Li, N. Hao, L. Tan, X. Meng, J. Ren, D. Chen and F. Tang, Nanoscale, 2012, 4, 3523-3529.

190.H. Liu, T. Liu, H. Wang, L. Li, L. Tan, C. Fu, G. Nie, D. Chen and F.

45 Tang, Biomaterials, 2013, 34, 6967-6975.

191.L. Tan, D. Chen, H. Liu and F. Tang, Adv. Mater., 2010, 22, 4885-4889.

192.S. H. Hu, Y. Y. Chen, T. C. Liu, T. H. Tung, D. M. Liu and S. Y. Chen, Chem. Commun., 2011, 47, 1776-1778.

193. Y. Sun, B. T. Mayers and Y. Xia, Nano Lett., 2002, 2, 481-485

50 194.W. Y. Li, X. Cai, C. H. Kim, G. R. Sun, Y. Zhang, R. Deng, M. X. Yang, J. Y. Chen, S. Achilefu, L. V. Wang and Y. N. Xia, Nanoscale, 2011, 3, 1724-1730.

195.M. S. Yavuz, Y. Cheng, J. Chen, C. M. Cobley, Q. Zhang, M. Rycenga, J. Xie, C. Kim, K. H. Song, A. G. Schwartz, L. V. Wang and Y. Xia, Nat.

55 Mater., 2009, 8, 935-939.

196. Z. Wang, Z. Chen, Z. Liu, P. Shi, K. Dong, E. Ju, J. Ren and X. Qu, Biomaterials, 2014, 35, 9678-9688.

197.P. Shi, M. Li, J. S. Ren and X. G. Qu, Adv. Funct. Mater., 2013, 23, 5412 5419.

60 198.G. D. Moon, S. W. Choi, X. Cai, W. Li, E. C. Cho, U. Jeong, L. V. Wang and Y. Xia, J. Am. Chem. Soc., 2011, 133, 4762-4765.
199.L. Tian, N. Gandra and S. Singamaneni, ACS Nano, 2013, 7, 4252-4260. 200.M. P. Melancon, M. Zhou and C. Li, Acc. Chem. Res., 2011, 44, 947-956.

201.B. G. Prevo, S. A. Esakoff, A. Mikhailovsky and J. A. Zasadzinski, Small, 2008, 4, 1183-1195.

202.H. Liu, T. Liu, X. Wu, L. Li, L. Tan, D. Chen and F. Tang, Adv. Mater., 2012, 24, 755-761.

203.M. S. Noh, S. Lee, H. Kang, J.-K. Yang, H. Lee, D. Hwang, J. W. Lee, S. Jeong, Y. Jang and B.-H. Jun, Biomaterials, 2015, 45, 81-92.

70 204.Q. Y. Bao, N. Zhang, D. D. Geng, J. W. Xue, M. Merritt, C. Zhang and Y. Ding, Int. J. Pharm., 2014, 477, 408-415.

205.T. Lajunen, L. Viitala, L. S. Kontturi, T. Laaksonen, H. Liang, E. Vuorimaa-Laukkanen, T. Viitala, X. Le Guével, M. Yliperttula and L. Murtomäki, J. Control. Release, 2015.

75 206.A. K. Rengan, M. Jagtap, A. De, R. Banerjee and R. Srivastava, Nanoscale, 2014, 6, 916-923.

207.S. J. Leung, M. C. Bobnick and M. Romanowski, Proc. Soc. Photo Opt. Instrum. Eng., 2010, 7577, 75770S.

208.Y. Ma, X. L. Liang, S. Tong, G. Bao, Q. S. Ren and Z. F. Dai, Adv. Funct. 80 Mater., 2012, 23, 815-822

209.J. You, R. Shao, X. Wei, S. Gupta and C. Li, Small, 2010, 6, 1022-1031.

210.J. You, G. Zhang and C. Li, ACS Nano, 2010, 4, 1033-1041.

211.J. You, R. Zhang, G. Zhang, M. Zhong, Y. Liu, C. S. Van Pelt, D. Liang, W. Wei, A. K. Sood and C. Li, J. Control. Release, 2012, 158, 319-328.

85 212.Y. Jin and X. Gao, J. Am. Chem. Soc., 2009, 131, 17774-17776.

213.J. Song, L. Cheng, A. Liu, J. Yin, M. Kuang and H. Duan, J. Am. Chem. Soc., 2011, 133, 10760-10763.

214.J. Song, J. Zhou and H. Duan, J. Am. Chem. Soc., 2012, 134, 13458-13469.

215.J. Song, L. Pu, J. Zhou, B. Duan and H. Duan, ACS Nano, 2013, 7, $9947-$ 9960.

216.J. Song, Z. Fang, C. Wang, J. Zhou, B. Duan, L. Pu and H. Duan, Nanoscale, 2013, 5, 5816-5824.

217. E. Bringas, O. Koysuren, D. V. Quach, M. Mahmoudi, E. Aznar, J. D. Roehling, M. D. Marcos, R. Martinez-Manez and P. Stroeve, Chem. Commun., 2012, 48, 5647-5649.

218.S. D. Kong, M. Sartor, C. M. Hu, W. Zhang, L. Zhang and S. Jin, Acta Biomater., 2013, 9, 5447-5452.

219.S. H. Hu, S. Y. Chen and X. Gao, ACS Nano, 2012, 6, 2558-2565.

220.C. C. Chen, Y. P. Lin, C. W. Wang, H. C. Tzeng, C. H. Wu, Y. C. Chen,

100 C. P. Chen, L. C. Chen and Y. C. Wu, J. Am. Chem. Soc., 2006, 128, 37093715 .

221.L. Zhao, J. Peng, Q. Huang, C. Li, M. Chen, Y. Sun, Q. Lin, L. Zhu and F. Li, Adv. Funct. Mater., 2014, 24, 363-371.

222.Y. Yang, F. Liu, X. Liu and B. Xing, Nanoscale, 2013, 5, 231-238.

105 223.J. V. Garcia, J. Yang, D. Shen, C. Yao, X. Li, R. Wang, G. D. Stucky, D. Zhao, P. C. Ford and F. Zhang, Small, 2012, 8, 3800-3805.

224.C. J. Carling, F. Nourmohammadian, J. C. Boyer and N. R. Branda, Angew. Chem. Int. Ed., 2010, 49, 3782-3785.

225.N. C. Fan, F. Y. Cheng, J. A. Ho and C. S. Yeh, Angew. Chem. Int. Ed., $110 \quad 2012, \mathbf{5 1}, 8806-8810$.

226.Y. Yang, Q. Shao, R. Deng, C. Wang, X. Teng, K. Cheng, Z. Cheng, L. Huang, Z. Liu, X. Liu and B. Xing, Angew. Chem. Int. Ed., 2012, 51, 3125-3129.

227.L. L. Fedoryshin, A. J. Tavares, E. Petryayeva, S. Doughan and U. J. Krull, ACS Appl. Mater. Interfaces, 2014, 6, 13600-13606.

228.A. H. Negussie, P. S. Yarmolenko, A. Partanen, A. Ranjan, G. Jacobs, D. Woods, H. Bryant, D. Thomasson, M. W. Dewhirst, B. J. Wood and M. R. Dreher, Int. J. Hyperthermia, 2011, 27, 140-155.

229.A. Partanen, P. S. Yarmolenko, A. Viitala, S. Appanaboyina, D. 20 Haemmerich, A. Ranjan, G. Jacobs, D. Woods, J. Enholm, B. J. Wood and M. R. Dreher, Int. J. Hyperthermia, 2012, 28, 320-336.

230.J. F. Gohy and Y. Zhao, Chem. Soc. Rev., 2013, 42, 7117-7129. 
231.H. Yan, C. Teh, S. Sreejith, L. Zhu, A. Kwok, W. Fang, X. Ma, K. T. Nguyen, V. Korzh and Y. Zhao, Angew. Chem. Int. Ed., 2012, 51, 83738377.

232.M. Haase and H. Schafer, Angew. Chem. Int. Ed. Engl, 2011, 50, 5808$5 \quad 5829$

233.Z. Gu, L. Yan, G. Tian, S. Li, Z. Chai and Y. Zhao, Adv. Mater., 2013, 25, 3758-3779.

234. J. Liu, W. Bu, L. Pan and J. Shi, Angew. Chem. Int. Ed., 2013, 52, 43754379.

10 235. L. Zhou, Z. Chen, K. Dong, M. Yin, J. Ren, X. Qu, Adv. Mater., 2014, 26, 2424-2430.

236.M. A. C. Stuart, W. T. Huck, J. Genzer, M. Müller, C. Ober, M. Stamm, G. B. Sukhorukov, I. Szleifer, V. V. Tsukruk and M. Urban, Nat. Mater., 2010, 9, 101-113.

15 237.M. Pernia Leal, A. Torti, A. Riedinger, R. La Fleur, D. Petti, R. Cingolani, R. Bertacco and T. Pellegrino, ACS Nano, 2012, 6, 10535-10545.

238.T. Y. Liu, S. H. Hu, K. H. Liu, R. S. Shaiu, D. M. Liu and S. Y. Chen, Langmuir, 2008, 24, 13306-13311.

239.A. Yavlovich, B. Smith, K. Gupta, R. Blumenthal and A. Puri, Mol. 20 Membr. Biol., 2010, 27, 364-381.

240.N. Fomina, C. McFearin, M. Sermsakdi, O. Edigin and A. Almutairi, J. Am. Chem. Soc., 2010, 132, 9540-9542.

241.S. H. Hu, S. Y. Chen, D. M. Liu and C. S. Hsiao, Adv. Mater., 2008, 20, 2690-2695.

25 242.S. H. Hu, D. M. Liu, T. H. Tung, F. L. Chen and S. Y. Chen, Adv. Funct. Mater., 2008, 18, 2946-2955.

243.P. Roy and A. Shahiwala, J. Control. Release, 2009, 134, 74-80.

244.A. Kikuchi and T. Okano, Adv. Drug Deliv. Rev., 2002, 54, 53-77.

245.Y. Qiu and K. Park, Adv. Drug Deliv. Rev., 2001, 53, 321-339.

30 246.N. S. Satarkar and J. Z. Hilt, J. Control. Release, 2008, 130, 246-251.

247.W. L. Chiang, C. J. Ke, Z. X. Liao, S. Y. Chen, F. R. Chen, C. Y. Tsai, Y. Xia and H. W. Sung, Small, 2012, 8, 3584-3588.

248.S. H. Hu, T. Y. Liu, D. M. Liu, S. Y. Chen, G. Sun, C. Cheng and K. L. Wooley, Macromolecules, 2007, 40, 6786-6788.

35 249.W. C. Huang, S. H. Hu, K. H. Liu, S. Y. Chen and D. M. Liu, J. Control. Release, 2009, 139, 221-228.

250.C. S. Kumar and F. Mohammad, Adv. Drug Deliv. Rev., 2011, 63, 789808.

251.J. P. May and S. D. Li, Expert Opin. Drug Deliv., 2013, 10, 511-527.

40 252.W. Rao, Z. S. Deng and J. Liu, Crit. Rev. Biomed. Eng., 2010, 38, 101-116.

253.O. Veiseh, J. W. Gunn and M. Zhang, Adv. Drug Deliv. Rev., 2010, 62, 284-304.

254.P. Cherukuri, E. S. Glazer and S. A. Curley, Adv. Drug Deliv. Rev., 2010, 62, 339-345.

\section{Abbreviation list}

70 AMF Alternating magnetic fields

EMF Electromagnetic filed

NIR Near infrared

HIFU High-intensity focused ultrasound

DDS Drug delivery system

75 MPS Mononuclear phagocyte system

EPR Enhanced permeability and retention

MRI Magnetic resonance imaging

GNPs Gold nanoparticles

GNRs Gold nanorods

$80 \gamma-\mathrm{Fe}_{2} \mathrm{O}_{3}$ Maghemite

$\mathrm{Fe}_{3} \mathrm{O}_{4} \quad$ Magnetite

NPs Nanoparticles

MSNs Mesoporous silica nanoparticles

TSLs Thermo-sensitive liposomes

${ }_{85}$ UCNPs Upconverting nanoparticles

dsDNA Double-stranded DNA

ssDNA Single-stranded DNA

RNAi RNA interference

PNiPAAm Poly( $N$-isopropyl acrylamide)

90 PAAm Polyacrylamide

PEG Poly(ethylene glycol)

PCL Poly( $\varepsilon$-caprolactone)

PEO Poly(ethylene oxide)

PPO Poly(propylene oxide)

${ }_{95}$ PAA Poly(acrylic acid)

PVOH/PVA Poly(vinyl alcohol)

PLL Polylysine peptide

PNVCL Poly( $N$-vinylcaprolactam)

CMRP Cobalt-mediated radical polymerization

100 RAFT Reversible addition-fragmentation transfer

ATRP Atom transfer radical polymerization

SIP Surface-initiated polymerization

LCST Lower critical solution temperature

DPPC 1,2-Dipalmitoyl-sn-glycerophosphocholine

105 DSPC 1,2-Distearoyl-sn-glycerophosphocholine

DOX Doxorubicin

SPR Surface plasmon resonance

TEM Transmission electron microscopy

PyCOOH Pyrrole-3-carboxylic acid

110 APS Ammonium persulfate

bp Basepair

SERS Surface enhanced Raman spectroscopy

RCL Reversibly cross-linking

R6G Rhodamine 6G

115 IS Ionic strength

MB Methylene blue

EDC 1-ethyl-3-(3-dimethylaminopropyl) carbodiimide

IgG Immunoglobulin G

PCM Phase-changing materials

${ }_{120} \mathrm{Tf} \quad$ Transferrin 


\section{Biography \& photography of authors}

\section{Table of Content}

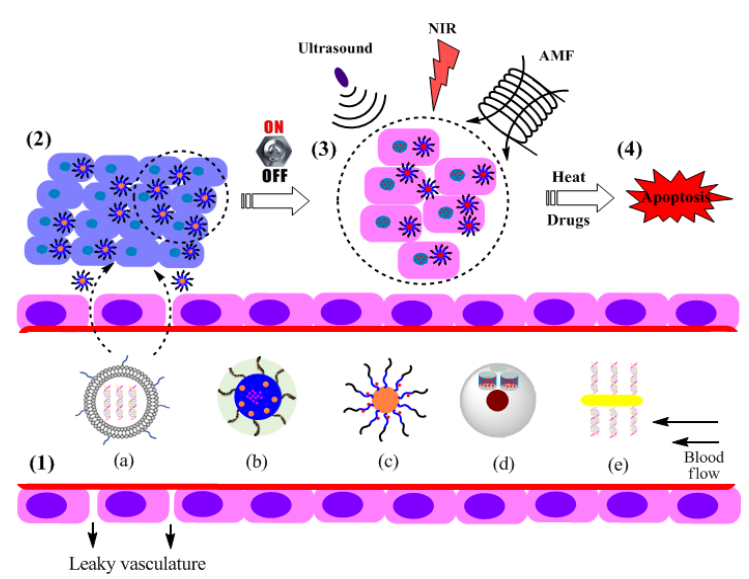

Scheme: (1) Hybrid nanovehicles with different kinds of structures 5 designed for drug delivery systems, such as liposomes (a), cross-linked polymer nanogels or micelles (b), organic/inorganic core/corona nanohybrids (c), mesoporous silica-based nanovehicles engineered with gating moieties (d) and host/guest nano-conjugates (e); (2) delivery of the guest molecules to desirable tumour sites via passive or active targeting 10 through the vasculature; (3) trigger release of the loaded cargos through remote stimuli, e.g. ultrasound, light (such as near infrared laser) or alternating magnetic field (AMF); (4) tumour cell apoptosis resulting from the synergistic effect of thermal and chemotherapeutic contributions.

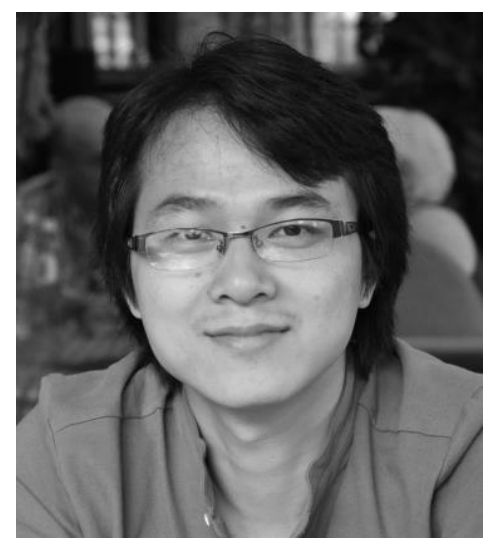

Ji Liu obtained his PhD in Nov. 2013 under the co-supervision of ${ }_{30}$ Prof. Christine Jérôme (University of Liège) and Prof. Etienne Duguet (University of Bordeaux). His PhD research was focused on developing innovated drug delivery systems based on specifically-designed macromolecules and inorganic nanocolloids for controlled release. After a short post-doc experience in the 35 same group of Prof. Jérôme, he joined the Melville Lab for Polymer Synthesis with Prof. Oren Scherman in University of Cambridge as a post-doc research associate since June 2014. Currently, his research is mostly focused on cucurbituril-based supramolecular host/guest interaction for polymeric self40 assembly and interfacial adhesion.

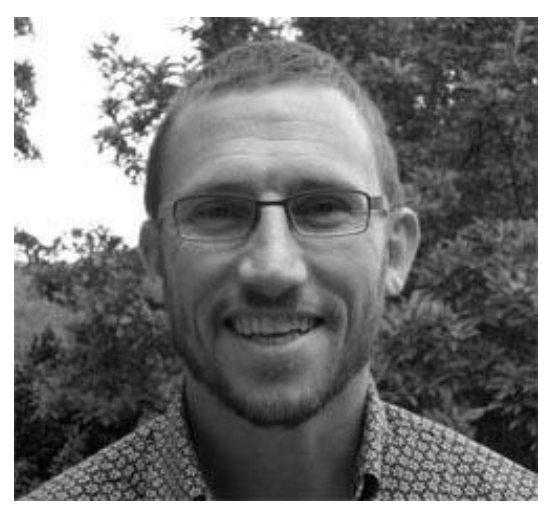

Dr. Christophe Detrembleur obtained his PhD in 2001 under the supervision of Prof. Robert Jérôme in CERM at University of ${ }_{45}$ Liège (Belgium). After 2.5-years as research scientist in Bayer AG (Leverkusen, Germany), he joined CERM in 2003 and currently works as a Research Director by National Funds for Scientific Research (F.R.S.-FNRS). His main research projects are in the field of the development of new controlled radical 50 polymerization techniques, the preparation of new multifunctional polymers and contribution of macromolecular engineering to nanotechnology, etc. 


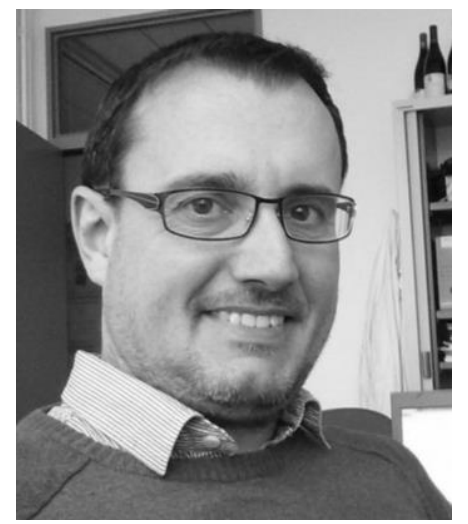

Stéphane Mornet received his $\mathrm{PhD}$ in physico-chemistry of 5 condensed matter at the University of Bordeaux in 2002. After 5 years of post-doctoral positions at the European Institute of Chemistry and Biology of Bordeaux, and at the Institute for Health and Consumer Protection (European Commission, Ispra, Italy), he is currently research fellow at the French National ${ }_{10}$ Centre for Scientific Research (CNRS) at the Institute of Condensed Matter Chemistry of Bordeaux (group 5 « Chemistry of nanomaterials »). His research, at the interface of chemistry and biology, focuses on the synthesis of inorganic nanoparticles, their surface functionalization and conjugation with biomolecules 15 for bioimaging and medical applications.

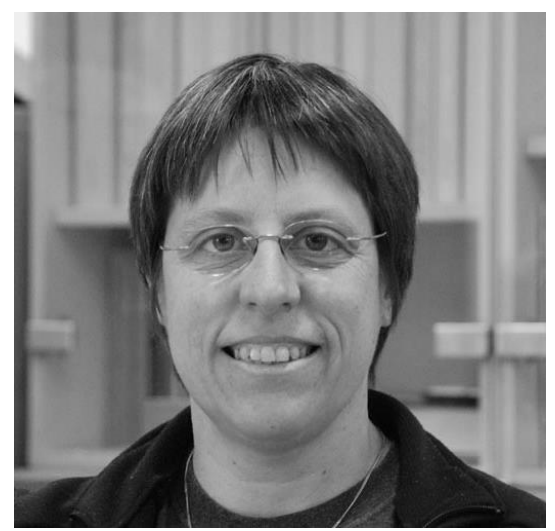

Christine Jérôme completed her PhD degree in 1998 (supervisor: Prof. Robert Jérôme) in CERM at the University of Liège. In 20 2000, she joined the University of Ulm in Germany as a recipient of the Humboldt scholarship. She is now Full Professor at the Chemistry Department of the University of Liege and director of CERM since 2006. Her research interests include electropolymerization, polymer functionalized nanoparticles and 25 biomaterials.

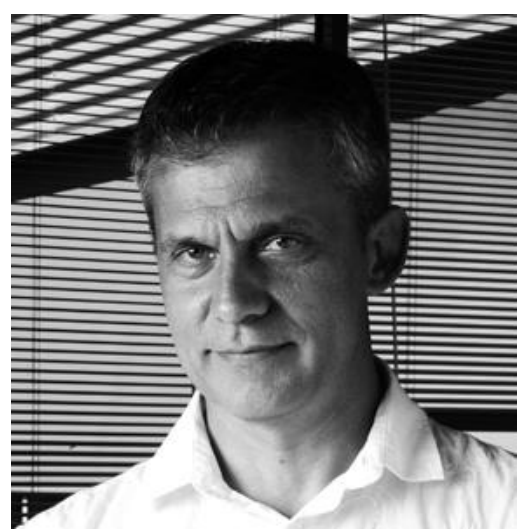

Dr. Etienne Duguet is Full Professor in Materials Chemistry at the University of Bordeaux, head of the research group ${ }_{30}$ "Chemistry of Nanomaterials" and deputy director of the CNRS Institute of Condensed Matter Chemistry of Bordeaux. He received his Physical-Chemistry Engineering diploma from the Graduate School of Chemistry and Physics of Bordeaux in 1989 and his Doctorate in Polymer Chemistry from the University of ${ }_{35}$ Bordeaux in 1992. His research area covers hybrid organicinorganic nanomaterials: nanoparticle synthesis, surface functionalization, polymer encapsulation and Janus/patchy particles for self-assembly. The targeted applications concerns medicine (MRI contrast agents, hyperthermia mediators, 40 nanosystems for heat-triggered drug release) and optics (pigments, nanoresonators for metamaterials in the visible range). 